\title{
Photoredox-catalyzed remote difunctionalizations of alkenes to synthesize fluoroalkyl ketones with DMSO as oxidant
}

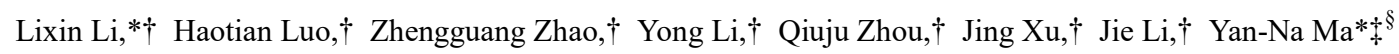
$\dagger$ College of Chemistry and Chemical Engineering, Xinyang Normal University, Xinyang, Henan 464000, China \$College of Chemistry and Molecular Engineering, Zhengzhou University, Zhengzhou, Henan 453000, China

${ }^{\S}$ School of Chemistry and Chemical Engineering, Henan Normal University, Xinxiang, Henan 453007, China

\section{Contents}

1. General information 2

2. Optimization of the reaction conditions 3

3. General procedures 3

4. Characterization of new compounds 4

5. Control and isotope labeling experiments 12

6. Spectra of new compounds 15 


\section{General information}

NMR Spectra was recorded on a JNM-ECZ00R/S3 ( ${ }^{1} \mathrm{H}$ NMR $600 \mathrm{MHz}$, ${ }^{13} \mathrm{C}$ NMR $150 \mathrm{MHz},{ }^{19} \mathrm{~F}$ NMR $564 \mathrm{MHz}$ ). Chemical shift are given in ppm. The spectra are calibrated to residual ${ }^{1} \mathrm{H}$ and ${ }^{13} \mathrm{C}$ singals of the solvents. Multiplicities are abbreviated as follows: singlet (s), doublet (d), triplet (t), quartet (q), doublet-doublet (dd), quintet (quint), septet (sept), multiplet (m), and broad (br). High-resolution electrospray ionization and electronic impact mass spectrometry was performed on a MicroMass Waters Xevo G2-XS QTof.

Materials and Methods: Unless otherwise stated, starting materials were purchased from Energy and Aldrich. Conversion was monitored by thin layer chromatography (TLC) using Merck TLC silica gel 60 F254. Compounds were visualized by UV light at $254 \mathrm{~nm}$ and by dipping the plates in an ehanolic vanillin/sulfuric acid solution or an aqueous potassium permanganate solution followed by heating. Flash column chromatography was performed over silica gel (200-400 mesh). Unless otherwise stated. Starting materials were according to previously reported procedures. 


\section{Optimization of the reaction conditions ${ }^{[a]}$}<smiles>C=CCc1ccccc1CC</smiles>

1 a

$$
+\mathrm{BrCF}_{2} \mathrm{CO}_{2} \mathrm{Et} \underset{\text { DMSO, Blue LED }}{\stackrel{\mathrm{PC}^{*}, \text { Base }}{\longrightarrow}}
$$

$2 a$<smiles></smiles>

$3 a$

\begin{tabular}{|c|c|c|c|}
\hline Entry & $\mathrm{PC}^{*}$ & Base & Yield $(\%)^{[b]}$ \\
\hline 1 & $f a c-\operatorname{Ir}(\mathrm{ppy})_{3}$ & $\mathrm{AgOAc}$ & 58 \\
\hline 2 & $f a c-\operatorname{Ir}(\mathrm{ppy})_{3}$ & AgTFA & 18 \\
\hline 3 & $f a c-\operatorname{Ir}(\mathrm{ppy})_{3}$ & $\mathrm{AgOBz}$ & 78 \\
\hline 4 & $f a c-\operatorname{Ir}(\mathrm{ppy})_{3}$ & AgOTf & 11 \\
\hline 5 & $f a c-\operatorname{Ir}(\mathrm{ppy})_{3}$ & AgOTs & 13 \\
\hline 6 & $f a c-\operatorname{Ir}(\mathrm{ppy})_{3}$ & $\mathrm{Ag}_{3} \mathrm{PO}_{4}$ & 52 \\
\hline 7 & $f a c-\operatorname{Ir}(\mathrm{ppy})_{3}$ & $\mathrm{Ag}_{2} \mathrm{CO}_{3}$ & 41 \\
\hline 8 & $f a c-\operatorname{Ir}(\mathrm{ppy})_{3}$ & $\mathrm{AgNO}_{3}$ & 30 \\
\hline $9^{[\mathrm{c}]}$ & $f a c-\operatorname{Ir}(\mathrm{ppy})_{3}$ & $\mathrm{Ag}_{3} \mathrm{PO}_{4}$ & 53 \\
\hline 10 & $\operatorname{Ir}\left[\mathrm{dF}\left(\mathrm{CF}_{3}\right)(\mathrm{ppy})\right]_{2}(\mathrm{dtbbpy}) \mathrm{PF}_{6}$ & $\mathrm{AgOBz}$ & 21 \\
\hline 11 & $\mathrm{Ru}(\mathrm{bpy})_{3} \cdot 6 \mathrm{H}_{2} \mathrm{O}$ & $\mathrm{AgOBz}$ & trace \\
\hline 12 & $\mathrm{RB}$ & $\mathrm{AgOBz}$ & 13 \\
\hline 13 & $\mathrm{EY}$ & $\mathrm{AgOBz}$ & 9 \\
\hline $14^{[\mathrm{d}]}$ & $f a c-\operatorname{Ir}(\mathrm{ppy})_{3}$ & $\mathrm{AgOBz}$ & NR \\
\hline $15^{[\mathrm{e}][\mathrm{f}][\mathrm{g}]}$ & -- & $\mathrm{AgOBz}$ & NR \\
\hline 16 & $f a c-\operatorname{Ir}(\mathrm{ppy})_{3}$ & $\mathrm{NEt}_{3}$ & NR \\
\hline 17 & $f a c-\operatorname{Ir}(\mathrm{ppy})_{3}$ & TMG & NR \\
\hline 18 & $f a c-\operatorname{Ir}(\mathrm{ppy})_{3}$ & DBU & NR \\
\hline 19 & $f a c-\operatorname{Ir}(\mathrm{ppy})_{3}$ & $\mathrm{Na}_{2} \mathrm{CO}_{3}$ & NR \\
\hline 20 & $f a c-\operatorname{Ir}(\mathrm{ppy})_{3}$ & $\mathrm{Cs}_{2} \mathrm{CO}_{3}$ & NR \\
\hline 21 & $f a c-\operatorname{Ir}(\mathrm{ppy})_{3}$ & $\mathrm{KH}_{2} \mathrm{PO}_{4}$ & NR \\
\hline 22 & $f a c-\operatorname{Ir}(\mathrm{ppy})_{3}$ & $\mathrm{~K}_{3} \mathrm{PO}_{4}$ & NR \\
\hline 23 & $f a c-\operatorname{Ir}(\mathrm{ppy})_{3}$ & $\mathrm{LiOH}$ & NR \\
\hline
\end{tabular}

[a] Reaction condition: 1a $(0.1 \mathrm{mmol}), \mathbf{2 a}(0.15 \mathrm{mmol}), \mathbf{P C}^{*}(2 \mathrm{~mol} \%),[\mathrm{Ag}](0.15 \mathrm{mmol})$, DMSO $(1.0 \mathrm{~mL})$, under $\mathrm{N}_{2}, 25{ }^{\circ} \mathrm{C}, 16 \mathrm{~h}$. [b] Yield determined by ${ }^{19} \mathrm{~F}$ NMP using $\mathrm{PhCF}_{3}$ as an internal standard. [c] $\mathrm{Ag}_{3} \mathrm{PO}_{4}(0.05 \mathrm{mmol})$ [d] no light. [e] no catalyst. [f] $\mathrm{NR}=$ no reaction. [g] AgOBz is silver benzoate.

\section{General procedures}

\subsection{General procedures for visible mediated oxidative fluoroalkylation reaction}


<smiles>C=CCc1ccccc1CC</smiles>

$1 \mathrm{a}$

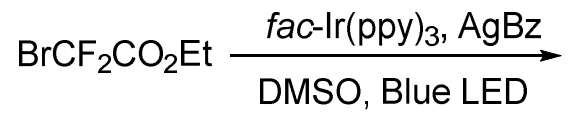

2a

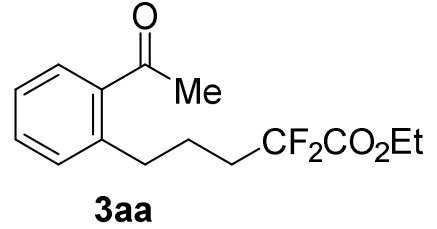

3aa

To an oven-dried $10 \mathrm{~mL}$ Schlenk tube was added $f a c-\operatorname{Ir}(\mathrm{ppy})_{3}(2 \mathrm{~mol} \%)$ and $\mathrm{AgBz}$ (1.5 equiv). The tube was evacuated and back-filled with nitrogen (this process was repeated three times), 1a $(0.2 \mathrm{mmol}, 29.2 \mathrm{mg}), \mathbf{2 a}(0.3 \mathrm{mmol}, 60.3 \mathrm{mg})$ and DMSO $(2.0 \mathrm{~mL})$ was added subsequently via syring. The resulting mixture was stirred at room temperature with $12 \mathrm{~W}$ irradiation until the reaction completion. The mixture was diluted with EA $(2.0 \mathrm{~mL} \times 3)$, organic phase was washed with saturated $\mathrm{NaCl}$ solution, dired over anhydrous $\mathrm{Na}_{2} \mathrm{SO}_{4}$, filtered and concentrated under reduced pressure. The crude mixture was purified by column chromatography on silica gel with PE/EA (20:1 to 5:1) as eluent to give 3aa (44.9 $\mathrm{mg}$, 79\% yield).

\section{Procedure for Gram-scale reaction:}

To an oven-dried $100 \mathrm{~mL}$ Schlenk round flask was added $f a c-\operatorname{Ir}(\mathrm{ppy})_{3}(1 \mathrm{~mol} \%)$ and $\mathrm{AgBz}(1.5$ equiv). The tube was evacuated and back-filled with nitrogen (this process was repeated three times), 1a $(5.0 \mathrm{mmol}, 730.0 \mathrm{mg}), \mathbf{2 a}(7.5 \mathrm{mmol}, 1.51 \mathrm{~g})$ and DMSO $(25.0 \mathrm{~mL})$ was added subsequently via syring. The resulting mixture was stirred at room temperature with $12 \mathrm{~W}$ irradiation until the reaction completion about 48 hours. The mixture was diluted with EA (10.0 $\mathrm{mL} \times 3$ ), organic phase was washed with saturated $\mathrm{NaCl}$ solution, dired over anhydrous $\mathrm{Na}_{2} \mathrm{SO}_{4}$, filtered and concentrated under reduced pressure. The crude mixture was purified by column chromatography on silica gel with PE/EA (20:1 to 5:1) as eluent to give 3aa (937.2 $\mathrm{mg}, 66 \%$ yield).

\section{Characterization of new compounds}<smiles>CCOC(=O)CCCc1ccccc1C(C)=O</smiles>

ethyl 5-(2-acetylphenyl)-2,2-difluoropentanoate (3aa):

Colorless oil, The crude mixture was purified by column chromatography on silica gel with PE/EA (20:1 to 10:1) as eluent to give $44.9 \mathrm{mg}$, (79\% yield). ${ }^{1} \mathrm{H}$ NMR $\left(600 \mathrm{MHz}, \mathrm{CDCl}_{3}\right) \delta 7.70$ $7.69(\mathrm{~m}, 1 \mathrm{H}), 7.43-7.40(\mathrm{~m}, 1 \mathrm{H}), 7.29$ (t, $J=7.4 \mathrm{~Hz}, 1 \mathrm{H}), 7.25(\mathrm{~d}, J=7.6 \mathrm{~Hz}, 1 \mathrm{H}), 4.33$ (q, $J=$ $7.0 \mathrm{~Hz}, 2 \mathrm{H}), 2.91(\mathrm{t}, J=7.7 \mathrm{~Hz}, 2 \mathrm{H}), 2.58(\mathrm{~s}, 3 \mathrm{H}), 2.14-2.11(\mathrm{~m}, 2 \mathrm{H}), 1.78-1.75(\mathrm{~m}, 2 \mathrm{H}), 1.33$ $(\mathrm{t}, J=7.4 \mathrm{~Hz}, 3 \mathrm{H}) ;{ }^{19} \mathrm{~F}$ NMR $\left(564 \mathrm{MHz}, \mathrm{CDCl}_{3}\right) \delta-105.73(\mathrm{t}, J=14.6 \mathrm{~Hz}, 2 \mathrm{~F}) ;{ }^{13} \mathrm{C}$ NMR $(150$ $\left.\mathrm{MHz}, \mathrm{CDCl}_{3}\right) \delta 201.61,164.31(\mathrm{t}, J=32.9 \mathrm{~Hz}), 141.49,137.43,131.67,131.23,129.61,126.20$, $116.25(\mathrm{t}, J=248.6 \mathrm{~Hz}), 62.73,34.21(\mathrm{t}, J=23.0 \mathrm{~Hz}), 33.24,29.68,23.39,13.93$; HRMS (ESI) $\mathrm{m} / \mathrm{z}$ calcd for $\mathrm{C}_{15} \mathrm{H}_{19} \mathrm{~F}_{2} \mathrm{O}_{3}\left[\mathrm{M}+\mathrm{H}^{+}\right]$285.1297, found 285.1291.<smiles>CCOC(=O)CCCc1ccccc1C(=O)c1ccccc1</smiles> 
ethyl 5-(2-benzoylphenyl)-2,2-difluoropentanoate (3ba):

Colorless oil, The crude mixture was purified by column chromatography on silica gel with PE/EA (20:1 to 10:1) as eluent to give $56.7 \mathrm{mg},\left(82 \%\right.$ yield). ${ }^{1} \mathrm{H}$ NMR $\left(600 \mathrm{MHz}, \mathrm{CDCl}_{3}\right) \delta 7.79$ $\left(\mathrm{dd}, J_{1}=8.0 \mathrm{~Hz}, J_{2}=1.0 \mathrm{~Hz}, 2 \mathrm{H}\right), 7.60-7.58(\mathrm{~m}, 1 \mathrm{H}), 7.47-7.42(\mathrm{~m}, 3 \mathrm{H}), 7.33-7.27(\mathrm{~m}, 3 \mathrm{H})$, $4.27(\mathrm{q}, J=7.0 \mathrm{~Hz}, 2 \mathrm{H}), 2.73(\mathrm{t}, J=7.8 \mathrm{~Hz}, 2 \mathrm{H}), 2.07-1.98(\mathrm{~m}, 2 \mathrm{H}), 1.79-1.73(\mathrm{~m}, 2 \mathrm{H}), 1.29(\mathrm{t}$, $J=7.0 \mathrm{~Hz}, 3 \mathrm{H}) ;{ }^{19} \mathrm{~F}$ NMR $\left(564 \mathrm{MHz}, \mathrm{CDCl}_{3}\right) \delta-105.78(\mathrm{t}, J=15.2 \mathrm{~Hz}, 2 \mathrm{~F}) ;{ }^{13} \mathrm{C} \mathrm{NMR}(150 \mathrm{MHz}$, $\left.\mathrm{CDCl}_{3}\right) \delta 198.33,164.18(\mathrm{t}, J=32.8 \mathrm{~Hz}), 140.21,137.71,133.25,130.43,130.15,130.09,128.87$, 128.44, 125.60, , 116.10 (t, $J=248.9 \mathrm{~Hz}), 62.73,34.01(\mathrm{t}, J=23.0 \mathrm{~Hz}), 32.36,23.35,13.89$; HRMS (ESI) $\mathrm{m} / \mathrm{z}$ calcd for $\mathrm{C}_{20} \mathrm{H}_{21} \mathrm{~F}_{2} \mathrm{O}_{3}\left[\mathrm{M}+\mathrm{H}^{+}\right] 347.1453$, found 347.1452.<smiles>CCOC(=O)CCCCc1ccccc1C(=O)c1cccc(C)c1</smiles>

ethyl 2,2-difluoro-5-(2-(3-methylbenzoyl)phenyl)pentanoate (3ca):

Colorless oil, The crude mixture was purified by column chromatography on silica gel with PE/EA (20:1 to 10:1) as eluent to give $63.4 \mathrm{mg},\left(88 \%\right.$ yield). ${ }^{1} \mathrm{H}$ NMR $\left(600 \mathrm{MHz}, \mathrm{CDCl}_{3}\right) \delta 7.62$ (s, 1H), 7.55 (d, $J=7.7 \mathrm{~Hz}, 1 \mathrm{H}), 7.45-7.39$ (m, 2H), $7.34-7.27$ (m, 4H), 4.27 (q, $J=7.4 \mathrm{~Hz}$, 2H), $2.73(\mathrm{t}, J=7.7 \mathrm{~Hz}, 2 \mathrm{H}), 2.40(\mathrm{~s}, 3 \mathrm{H}), 2.06-1.98(\mathrm{~m}, 2 \mathrm{H}), 1.79-1.73(\mathrm{~m}, 2 \mathrm{H}), 1.29(\mathrm{t}, J=$ $7.3 \mathrm{~Hz}, 3 \mathrm{H}) ;{ }^{19} \mathrm{~F}$ NMR $\left(564 \mathrm{MHz}, \mathrm{CDCl}_{3}\right) \delta-105.80(\mathrm{t}, J=17.2 \mathrm{~Hz}, 2 \mathrm{~F}) ;{ }^{13} \mathrm{C}$ NMR $(150 \mathrm{MHz}$, $\left.\mathrm{CDCl}_{3}\right) \delta 198.55,164.17(\mathrm{t}, J=32.9 \mathrm{~Hz}), 140.10,138.54,138.33,137.70,134.06,130.41,130.33$, 130.02, 128.80, 128.28, 127.57, 125.57, 116.09 (t, $J=248.5 \mathrm{~Hz}), 62.71,34.00$ (t, $J=23.1 \mathrm{~Hz}$ ), 32.33, 23.31, 21.26, 13.87; HRMS (ESI) $\mathrm{m} / \mathrm{z}$ calcd for $\mathrm{C}_{21} \mathrm{H}_{23} \mathrm{~F}_{2} \mathrm{O}_{3}\left[\mathrm{M}+\mathrm{H}^{+}\right] 361.1610$, found 361.1606 .<smiles>CCOC(=O)CCCc1ccccc1C(=O)c1ccc(F)cc1</smiles>

ethyl 2,2-difluoro-5-(2-(4-fluorobenzoyl)phenyl)pentanoate (3da):

Colorless oil, The crude mixture was purified by column chromatography on silica gel with PE/EA (20:1 to 10:1) as eluent to give $58.9 \mathrm{mg},\left(81 \%\right.$ yield). ${ }^{1} \mathrm{H}$ NMR $\left(600 \mathrm{MHz}, \mathrm{CDCl}_{3}\right) \delta 7.83$ $\left(\mathrm{dd}, J_{1}=8.8 \mathrm{~Hz}, J_{2}=5.5 \mathrm{~Hz}, 2 \mathrm{H}\right), 7.45-7.43(\mathrm{~m}, 1 \mathrm{H}), 7.33(\mathrm{~d}, J=7.7 \mathrm{~Hz}, 1 \mathrm{H}), 7.28-7.27(\mathrm{~m}$, 2H), 7.13 (t, $J=8.5 \mathrm{~Hz}, 2 \mathrm{H}), 4.28$ (q, $J=7.3 \mathrm{~Hz}, 2 \mathrm{H}), 2.73$ (t, $J=7.7 \mathrm{~Hz}, 2 \mathrm{H}), 2.07-1.98$ (m, $2 \mathrm{H}), 1.78-1.72(\mathrm{~m}, 2 \mathrm{H}), 1.30(\mathrm{t}, J=7.1 \mathrm{~Hz}, 3 \mathrm{H}) ;{ }^{19} \mathrm{~F} \mathrm{NMR}\left(564 \mathrm{MHz}, \mathrm{CDCl}_{3}\right) \delta-104.47(\mathrm{~s}, 1 \mathrm{~F})$, $-105.85(\mathrm{t}, J=17.4 \mathrm{~Hz}, 2 \mathrm{~F}) ;{ }^{13} \mathrm{C}$ NMR $\left(150 \mathrm{MHz}, \mathrm{CDCl}_{3}\right) \delta 196.73,166.69(\mathrm{~d}, J=254.1 \mathrm{~Hz})$, 164.17 (t, $J=32.2 \mathrm{~Hz}), 140.12,138.06,138.04,132.84$ (d, $J=9.2 \mathrm{~Hz}), 130.53,130.19,128.62$, 125.68, 116.06 (t, $J=248.6 \mathrm{~Hz}), 115.71$ (d, $J=21.7 \mathrm{~Hz}), 62.76,33.97$ (t, $J=23.1 \mathrm{~Hz}), 32.32$, 23.33, 13.89; HRMS (ESI) $\mathrm{m} / \mathrm{z}$ calcd for $\mathrm{C}_{20} \mathrm{H}_{20} \mathrm{~F}_{3} \mathrm{O}_{3}\left[\mathrm{M}+\mathrm{H}^{+}\right]$365.1359, found 365.1352.<smiles>CCOC(=O)CCCc1ccccc1C(=O)c1ccc(Cl)cc1</smiles>

ethyl 5-(2-(4-chlorobenzoyl)phenyl)-2,2-difluoropentanoate (3ea):

Colorless oil, The crude mixture was purified by column chromatography on silica gel with 
PE/EA (20:1 to 10:1) as eluent to give $59.3 \mathrm{mg}$, (78\% yield). ${ }^{1} \mathrm{H}$ NMR (600 $\left.\mathrm{MHz}, \mathrm{CDCl}_{3}\right) \delta 7.74-$ $7.72(\mathrm{~m}, 2 \mathrm{H}), 7.46-7.43(\mathrm{~m}, 3 \mathrm{H}), 7.33(\mathrm{~d}, J=7.6 \mathrm{~Hz}, 1 \mathrm{H}), 7.28-7.27(\mathrm{~m}, 2 \mathrm{H}), 4.28(\mathrm{q}, J=7.1$ $\mathrm{Hz}, 2 \mathrm{H}), 2.72(\mathrm{t}, J=7.7 \mathrm{~Hz}, 2 \mathrm{H}), 2.07-1.99(\mathrm{~m}, 2 \mathrm{H}), 1.78-1.73(\mathrm{~m}, 2 \mathrm{H}), 1.30(\mathrm{t}, J=7.0 \mathrm{~Hz}$, $3 \mathrm{H}) ;{ }^{19} \mathrm{~F}$ NMR $\left(564 \mathrm{MHz}, \mathrm{CDCl}_{3}\right) \delta-105.84(\mathrm{t}, J=17.4 \mathrm{~Hz}, 2 \mathrm{~F}) ;{ }^{13} \mathrm{C} \mathrm{NMR}\left(150 \mathrm{MHz}, \mathrm{CDCl}_{3}\right) \delta$ $197.01,164.18(\mathrm{t}, J=32.3 \mathrm{~Hz}), 140.31,139.80,137.80,136.01,131.52,130.69,130.26,128.81$, 128.77, 125.71, 116.06 (t, $J=248.8 \mathrm{~Hz}), 62.78,33.97(\mathrm{t}, J=23.1 \mathrm{~Hz}), 32.34,23.38,13.90$; HRMS (ESI) $\mathrm{m} / \mathrm{z}$ calcd for $\mathrm{C}_{20} \mathrm{H}_{20} \mathrm{ClF}_{2} \mathrm{O}_{3}\left[\mathrm{M}+\mathrm{H}^{+}\right]$381.1064, found 381.1067 .<smiles>CCCC(CCCCC(=O)OCC)(C(=O)OCC)C(=O)c1ccccc1</smiles>

triethyl 1,1-difluoro-7-oxo-7-phenylheptane-1,6,6-tricarboxylate (3fa):

Colorless oil, The crude mixture was purified by column chromatography on silica gel with PE/EA (20:1 to 10:1) as eluent to give $64.5 \mathrm{mg}$, (73\% yield). ${ }^{1} \mathrm{H}$ NMR $\left(600 \mathrm{MHz}, \mathrm{CDCl}_{3}\right) \delta 7.83$ $(\mathrm{d}, J=7.6 \mathrm{~Hz}, 2 \mathrm{H}), 7.53$ (t, $J=7.3 \mathrm{~Hz}, 1 \mathrm{H}), 7.41(\mathrm{t}, J=7.9 \mathrm{~Hz}, 2 \mathrm{H}), 4.32(\mathrm{q}, J=7.0 \mathrm{~Hz}, 2 \mathrm{H})$, $4.23-4.14(\mathrm{~m}, 4 \mathrm{H}), 2.25(\mathrm{t}, J=9.2 \mathrm{~Hz}, 2 \mathrm{H}), 2.07-2.01(\mathrm{~m}, 2 \mathrm{H}), 1.50-1.49(\mathrm{~m}, 4 \mathrm{H}), 1.34(\mathrm{t}, J=$ $7.0 \mathrm{~Hz}, 3 \mathrm{H}), 1.12(\mathrm{t}, J=7.0 \mathrm{~Hz}, 6 \mathrm{H}) ;{ }^{19} \mathrm{~F} \mathrm{NMR}\left(564 \mathrm{MHz}, \mathrm{CDCl}_{3}\right) \delta-106.04(\mathrm{t}, J=17.0 \mathrm{~Hz}, 2 \mathrm{~F})$; ${ }^{13} \mathrm{C} \mathrm{NMR}\left(150 \mathrm{MHz}, \mathrm{CDCl}_{3}\right) \delta 192.04,167.84,164.26(\mathrm{t}, J=32.7 \mathrm{~Hz}), 135.86,132.96,128.64$, 128.36, 116.09 (t, $J=248.6 \mathrm{~Hz}), 68.25,62.78,62.02,34.06$ (t, $J=23.2 \mathrm{~Hz}), 34.01,24.35,21.69$, 13.94, 13.72; HRMS (ESI) m/z calcd for $\mathrm{C}_{22} \mathrm{H}_{29} \mathrm{~F}_{2} \mathrm{O}_{7}\left[\mathrm{M}+\mathrm{H}^{+}\right]$443.1876, found 443.1869.

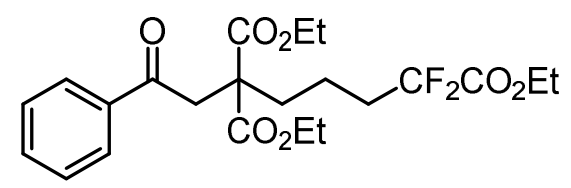

\section{triethyl 1,1-difluoro-8-oxo-8-phenyloctane-1,6,6-tricarboxylate (3ga):}

Colorless oil, The crude mixture was purified by column chromatography on silica gel with PE/EA (20:1 to 10:1) as eluent to give $69.8 \mathrm{mg}$, (79\% yield). ${ }^{1} \mathrm{H}$ NMR (600 MHz, $\left.\mathrm{CDCl}_{3}\right) \delta 7.97-$ $7.96(\mathrm{~m}, 2 \mathrm{H}), 7.59-7.57(\mathrm{~m}, 1 \mathrm{H}), 7.48-7.46(\mathrm{~m}, 2 \mathrm{H}), 4.27(\mathrm{q}, J=7.1 \mathrm{~Hz}, 2 \mathrm{H}), 4.22(\mathrm{q}, J=6.5$ $\mathrm{Hz}, 4 \mathrm{H}), 3.69(\mathrm{~s}, 2 \mathrm{H}), 2.16(\mathrm{t}, J=8.1 \mathrm{~Hz}, 2 \mathrm{H}), 2.08-2.00(\mathrm{~m}, 2 \mathrm{H}), 1.46-1.41(\mathrm{~m}, 2 \mathrm{H}), 1.29(\mathrm{t}, J$ $=7.0 \mathrm{~Hz}, 3 \mathrm{H}), 1.23(\mathrm{t}, J=7.4 \mathrm{~Hz}, 6 \mathrm{H}) ;{ }^{19} \mathrm{~F}$ NMR $\left(564 \mathrm{MHz}, \mathrm{DMSO}_{-} \mathrm{d}_{6}\right) \delta-104.91(\mathrm{t}, J=16.8 \mathrm{~Hz}$, 2F); ${ }^{13} \mathrm{C}$ NMR $\left(150 \mathrm{MHz}, \mathrm{CDCl}_{3}\right) \delta 196.48,170.53,164.04(\mathrm{t}, J=32.7 \mathrm{~Hz}), 136.38,133.45$, 128.64, 127.99, $115.76(\mathrm{t}, J=248.4 \mathrm{~Hz}), 62.78,61.66,55.16,41.23,34.48(\mathrm{t}, J=23.3 \mathrm{~Hz}), 17.02$, 13.93, 13.85; HRMS (ESI) $\mathrm{m} / \mathrm{z}$ calcd for $\mathrm{C}_{22} \mathrm{H}_{29} \mathrm{~F}_{2} \mathrm{O}_{7}\left[\mathrm{M}+\mathrm{H}^{+}\right]$443.1876, found 443.1878.

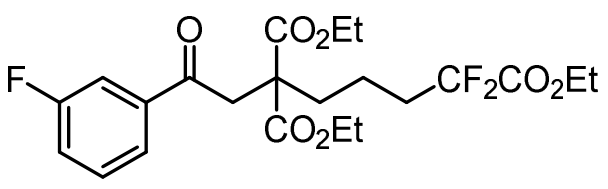

triethyl 1,1-difluoro-8-(3-fluorophenyl)-8-oxooctane-1,6,6-tricarboxylate (3ha):

Colorless oil, The crude mixture was purified by column chromatography on silica gel with PE/EA (20:1 to 10:1) as eluent to give $67.1 \mathrm{mg}$, (73\% yield). ${ }^{1} \mathrm{H}$ NMR $\left(600 \mathrm{MHz}, \mathrm{CDCl}_{3}\right) \delta 7.77$ $\left(\mathrm{d}, J_{1}=7.9 \mathrm{~Hz}, 1 \mathrm{H}\right), 7.65-7.63(\mathrm{~m}, 1 \mathrm{H}), 7.48-7.44(\mathrm{~m}, 1 \mathrm{H}), 7.30-7.27(\mathrm{~m}, 1 \mathrm{H}), 4.29(\mathrm{q}, J=7.3$ $\mathrm{Hz}, 2 \mathrm{H}), 4.23(\mathrm{q}, J=7.0 \mathrm{~Hz}, 4 \mathrm{H}), 3.65(\mathrm{~s}, 2 \mathrm{H}), 2.16(\mathrm{t}, J=8.6 \mathrm{~Hz}, 2 \mathrm{H}), 2.09-2.02(\mathrm{~m}, 2 \mathrm{H}), 1.46$ $-1.42(\mathrm{~m}, 2 \mathrm{H}), 1.31(\mathrm{t}, J=7.0 \mathrm{~Hz}, 3 \mathrm{H}), 1.24(\mathrm{t}, J=7.0 \mathrm{~Hz}, 6 \mathrm{H}) ;{ }^{19} \mathrm{~F} \mathrm{NMR}\left(564 \mathrm{MHz}, \mathrm{CDCl}_{3}\right) \delta$ $-106.16(\mathrm{t}, J=17.3 \mathrm{~Hz}, 2 \mathrm{~F}),-111.43(\mathrm{t}, J=7.7 \mathrm{~Hz}, 1 \mathrm{~F}) ;{ }^{13} \mathrm{C} \mathrm{NMR}\left(150 \mathrm{MHz}, \mathrm{CDCl}_{3}\right) \delta 195.34$, 
$170.40,164.06(\mathrm{t}, J=32.5 \mathrm{~Hz}), 163.66(\mathrm{~d}, J=246.6 \mathrm{~Hz}), 138.48(\mathrm{~d}, J=5.8 \mathrm{~Hz}), 130.41(\mathrm{~d}, J=$ $29.6 \mathrm{~Hz}), 123.82$ (d, $J=9.2 \mathrm{~Hz}), 120.58(\mathrm{~d}, J=21.3 \mathrm{~Hz}), 115.75$ (t, $J=248.7 \mathrm{~Hz}), 114.85$ (d, $J=$ $22.6 \mathrm{~Hz}), 62.83,61.78,55.22,41.42,34.47$ (t, $J=23.2 \mathrm{~Hz}), 32.47,17.04,13.95,13.89$; HRMS (ESI) $\mathrm{m} / \mathrm{z}$ calcd for $\mathrm{C}_{22} \mathrm{H}_{28} \mathrm{~F}_{3} \mathrm{O}_{7}\left[\mathrm{M}+\mathrm{H}^{+}\right]$461.1782, found 461.1770 .<smiles>CCOC(=O)[C-]CCCC(CC(=O)c1ccc(F)cc1)(OCC)C(=O)OCC</smiles>

\section{triethyl 1,1-difluoro-8-(4-fluorophenyl)-8-oxooctane-1,6,6-tricarboxylate (3ia):}

Colorless oil, The crude mixture was purified by column chromatography on silica gel with PE/EA (20:1 to 10:1) as eluent to give $74.5 \mathrm{mg}$, (81\% yield). ${ }^{1} \mathrm{H}$ NMR (600 MHz, $\left.\mathrm{CDCl}_{3}\right) \delta 8.01-$ $7.98(\mathrm{~m}, 2 \mathrm{H}), 7.15-7.12(\mathrm{~m}, 2 \mathrm{H}), 4.28(\mathrm{q}, J=7.1 \mathrm{~Hz}, 2 \mathrm{H}), 4.22-4.19(\mathrm{~m}, 4 \mathrm{H}), 3.65(\mathrm{~s}, 2 \mathrm{H})$, $2.16(\mathrm{t}, J=8.6 \mathrm{~Hz}, 2 \mathrm{H}), 2.08-2.00(\mathrm{~m}, 2 \mathrm{H}), 1.46-1.40(\mathrm{~m}, 2 \mathrm{H}), 1.31(\mathrm{t}, J=7.1 \mathrm{~Hz}, 3 \mathrm{H}), 1.23(\mathrm{t}$, $J=7.0 \mathrm{~Hz}, 6 \mathrm{H}) ;{ }^{19} \mathrm{~F}$ NMR $\left(564 \mathrm{MHz}, \mathrm{CDCl}_{3}\right) \delta-104.37(\mathrm{~s}, 1 \mathrm{~F}),-106.18(\mathrm{t}, J=17.3 \mathrm{~Hz}, 2 \mathrm{~F}) ;{ }^{13} \mathrm{C}$ NMR $\left(150 \mathrm{MHz}, \mathrm{CDCl}_{3}\right) \delta 194.93,170.48,166.77(\mathrm{~d}, J=103.6 \mathrm{~Hz}), 164.04(\mathrm{t}, J=32.5 \mathrm{~Hz})$, 132.85, 130.73 (d, $J=9.0 \mathrm{~Hz}$ ), 117.41 (t, $J=248.8 \mathrm{~Hz}$ ), 115.75 (d, $J=22.0 \mathrm{~Hz}$ ), 62.80, 61.71, 55.22, 41.14, $34.46(\mathrm{t}, J=23.1 \mathrm{~Hz}), 32.43,17.02,13.93,13.86$; HRMS (ESI) $\mathrm{m} / \mathrm{z}$ calcd for $\mathrm{C}_{22} \mathrm{H}_{28} \mathrm{~F}_{3} \mathrm{O}_{7}\left[\mathrm{M}+\mathrm{H}^{+}\right]$461.1782, found 461.1771 .

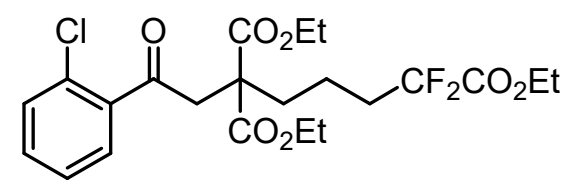

\section{triethyl 8-(2-chlorophenyl)-1,1-difluoro-8-oxooctane-1,6,6-tricarboxylate (3ja):}

Colorless oil, The crude mixture was purified by column chromatography on silica gel with PE/EA (20:1 to 10:1) as eluent to give $66.5 \mathrm{mg},\left(70 \%\right.$ yield). ${ }^{1} \mathrm{H}$ NMR $\left(600 \mathrm{MHz}, \mathrm{CDCl}_{3}\right) \delta 7.48-$ $7.47(\mathrm{~m}, 1 \mathrm{H}), 7.42-7.39(\mathrm{~m}, 2 \mathrm{H}), 7.38-7.31(\mathrm{~m}, 1 \mathrm{H}), 4.31$ (q, $J=7.2 \mathrm{~Hz}, 2 \mathrm{H}), 4.23$ (q, $J=7.0$ $\mathrm{Hz}, 4 \mathrm{H}), 3.63(\mathrm{~s}, 2 \mathrm{H}), 2.16(\mathrm{t}, J=8.6 \mathrm{~Hz}, 2 \mathrm{H}), 2.11-2.03(\mathrm{~m}, 2 \mathrm{H}), 1.49-1.45(\mathrm{~m}, 2 \mathrm{H}), 1.33$ (t, $J$ $=6.9 \mathrm{~Hz}, 3 \mathrm{H}), 1.25(\mathrm{t}, J=6.9 \mathrm{~Hz}, 6 \mathrm{H}) ;{ }^{19} \mathrm{~F} \mathrm{NMR}\left(564 \mathrm{MHz}, \mathrm{CDCl}_{3}\right) \delta-106.13(\mathrm{t}, J=16.2 \mathrm{~Hz}, 2 \mathrm{~F})$; ${ }^{13} \mathrm{C}$ NMR $\left(150 \mathrm{MHz}, \mathrm{CDCl}_{3}\right) \delta 199.64,170.27,164.08(\mathrm{t}, J=32.5 \mathrm{~Hz}), 138.81,131.92,130.68$, 130.54, 128.90, 126.99, 115.78 (t, $J=248.9 \mathrm{~Hz}), 62.85,61.78,55.55,45.54,34.51$ (t, $J=23.2 \mathrm{~Hz})$, 32.63, 17.07, 13.97, 13.91; HRMS (ESI) $\mathrm{m} / \mathrm{z}$ calcd for $\mathrm{C}_{22} \mathrm{H}_{28} \mathrm{ClF}_{2} \mathrm{O}_{7}\left[\mathrm{M}+\mathrm{H}^{+}\right]$477.1486, found 477.1477 .<smiles>CCOC(=O)CCCC(C)(CC(=O)c1cccc(Cl)c1)C(=O)OCC</smiles>

\section{triethyl 8-(3-chlorophenyl)-1,1-difluoro-8-oxooctane-1,6,6-tricarboxylate (3ka):}

Colorless oil, The crude mixture was purified by column chromatography on silica gel with PE/EA (20:1 to 10:1) as eluent to give $73.3 \mathrm{mg}$, (77\% yield). ${ }^{1} \mathrm{H}$ NMR (600 MHz, $\left.\mathrm{CDCl}_{3}\right) \delta 7.93$ (s, 1H), $7.85-7.84(\mathrm{~m}, 1 \mathrm{H}), 7.56-7.55(\mathrm{~m}, 1 \mathrm{H}), 7.43-7.41(\mathrm{~m}, 1 \mathrm{H}), 4.29$ (q, $J=7.1 \mathrm{~Hz}, 2 \mathrm{H})$, $4.23(\mathrm{q}, J=6.8 \mathrm{~Hz}, 4 \mathrm{H}), 3.64(\mathrm{~s}, 2 \mathrm{H}), 2.16(\mathrm{t}, J=8.5 \mathrm{~Hz}, 2 \mathrm{H}), 2.07-2.02(\mathrm{~m}, 2 \mathrm{H}), 1.45-1.42(\mathrm{~m}$, 2H), $1.31(\mathrm{t}, J=7.1 \mathrm{~Hz}, 3 \mathrm{H}), 1.24(\mathrm{t}, J=7.3 \mathrm{~Hz}, 6 \mathrm{H}) ;{ }^{19} \mathrm{~F} \mathrm{NMR}\left(564 \mathrm{MHz}, \mathrm{CDCl}_{3}\right) \delta-106.15(\mathrm{t}, J$ $=17.4 \mathrm{~Hz}, 2 \mathrm{~F}) ;{ }^{13} \mathrm{C} \mathrm{NMR}\left(150 \mathrm{MHz}, \mathrm{CDCl}_{3}\right) \delta 195.31,170.38,164.05(\mathrm{t}, J=32.8 \mathrm{~Hz}), 137.90$, $133.38,130.02,128.13,126.11,115.74(\mathrm{t}, J=248.8 \mathrm{~Hz}), 62.83,61.78,55.21,41.37,34.46$ (t, $J=$ 
23.2 Hz), 32.47, 17.04, 13.97, 13.88; HRMS (ESI) m/z calcd for $\mathrm{C}_{22} \mathrm{H}_{28} \mathrm{ClF}_{2} \mathrm{O}_{7}\left[\mathrm{M}+\mathrm{H}^{+}\right]$477.1486, found 477.1479 .<smiles>CCCC(CCCC(=O)OCC)(CC(=O)c1ccc(Cl)cc1)OCC</smiles>

\section{triethyl 8-(4-chlorophenyl)-1,1-difluoro-8-oxooctane-1,6,6-tricarboxylate (3la):}

Colorless oil, The crude mixture was purified by column chromatography on silica gel with PE/EA (20:1 to 10:1) as eluent to give $79.9 \mathrm{mg}$, (84\% yield). ${ }^{1} \mathrm{H}$ NMR $\left(600 \mathrm{MHz}, \mathrm{CDCl}_{3}\right) \delta 7.91$ $(\mathrm{d}, J=8.4 \mathrm{~Hz}, 2 \mathrm{H}), 7.45(\mathrm{~d}, J=8.7 \mathrm{~Hz}, 2 \mathrm{H}), 4.29$ (q, $J=7.0 \mathrm{~Hz}, 2 \mathrm{H}), 4.22(\mathrm{q}, J=7.8 \mathrm{~Hz}, 4 \mathrm{H})$, 4.19 (s, 2H), 2.17 (t, $J=8.6 \mathrm{~Hz}, 2 \mathrm{H}), 2.07-2.01(\mathrm{~m}, 2 \mathrm{H}), 1.44-1.41(\mathrm{~m}, 2 \mathrm{H}), 1.30$ (t, $J=7.4 \mathrm{~Hz}$, $3 \mathrm{H}), 1.23(\mathrm{t}, J=7.3 \mathrm{~Hz}, 6 \mathrm{H}) ;{ }^{19} \mathrm{~F}$ NMR $\left(564 \mathrm{MHz}, \mathrm{CDCl}_{3}\right) \delta-106.19(\mathrm{t}, J=17.3 \mathrm{~Hz}, 2 \mathrm{~F}) ;{ }^{13} \mathrm{C}$ NMR $\left(150 \mathrm{MHz}, \mathrm{CDCl}_{3}\right) \delta 195.34,170.43,164.04(\mathrm{t}, J=32.3 \mathrm{~Hz}), 139.96,134.72,129.43$, $128.98,115.74$ (t, $J=248.6 \mathrm{~Hz}), 62.81,61.74,55.22,41.20,34.45$ (t, $J=23.1 \mathrm{~Hz}), 32.45,17.02$, 13.94, 13.87; HRMS (ESI) $\mathrm{m} / \mathrm{z}$ calcd for $\mathrm{C}_{22} \mathrm{H}_{28} \mathrm{ClF}_{2} \mathrm{O}_{7}\left[\mathrm{M}+\mathrm{H}^{+}\right]$477.1486, found 477.1478.<smiles>CCCC(CCCCC(=O)OCC)(CC(=O)c1cccc(Br)c1)C(=O)OCC</smiles>

\section{triethyl 8-(3-bromophenyl)-1,1-difluoro-8-oxooctane-1,6,6-tricarboxylate (3ma):}

Colorless oil, The crude mixture was purified by column chromatography on silica gel with PE/EA (20:1 to 10:1) as eluent to give $82.1 \mathrm{mg}$, (79\% yield). ${ }^{1} \mathrm{H}$ NMR (600 MHz, $\left.\mathrm{CDCl}_{3}\right) \delta 8.09-$ $8.08(\mathrm{~m}, 1 \mathrm{H}), 7.90-7.88(\mathrm{~m}, 1 \mathrm{H}), 7.71-7.70(\mathrm{~m}, 1 \mathrm{H}), 7.36(\mathrm{t}, J=7.7 \mathrm{~Hz}, 1 \mathrm{H}), 4.30(\mathrm{q}, J=7.3$ $\mathrm{Hz}, 2 \mathrm{H}), 4.23-4.19(\mathrm{~m}, 4 \mathrm{H}), 3.64(\mathrm{~s}, 2 \mathrm{H}), 2.15(\mathrm{t}, J=8.5 \mathrm{~Hz}, 2 \mathrm{H}), 2.08-2.02(\mathrm{~m}, 2 \mathrm{H}), 1.46-$ $1.42(\mathrm{~m}, 2 \mathrm{H}), 1.31(\mathrm{t}, J=7.4 \mathrm{~Hz}, 3 \mathrm{H}), 1.24(\mathrm{t}, J=7.4 \mathrm{~Hz}, 6 \mathrm{H}) ;{ }^{19} \mathrm{~F}$ NMR $\left(564 \mathrm{MHz}, \mathrm{CDCl}_{3}\right) \delta$ $-106.14(\mathrm{t}, J=17.3 \mathrm{~Hz}, 2 \mathrm{~F}) ;{ }^{13} \mathrm{C}$ NMR $\left(150 \mathrm{MHz}, \mathrm{CDCl}_{3}\right) \delta 195.23,170.37,164.05(\mathrm{t}, J=33.2$ $\mathrm{Hz}), 138.10,136.31,131.08,130.27,126.55,123.04,115.75$ ( $\mathrm{t}, J=249.0 \mathrm{~Hz}), 62.84,61.79,55.21$, 41.35, $34.46(\mathrm{t}, J=23.7 \mathrm{~Hz}), 32.48,17.04,13.95,13.89$; HRMS (ESI) $\mathrm{m} / \mathrm{z}$ calcd for $\mathrm{C}_{22} \mathrm{H}_{28} \mathrm{BrF}_{2} \mathrm{O}_{7}\left[\mathrm{M}+\mathrm{H}^{+}\right]$521.0981, found 521.0973.

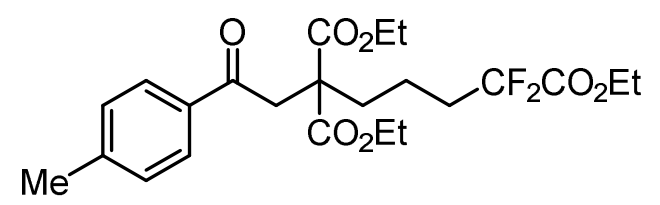

\section{triethyl 1,1-difluoro-8-oxo-8-(p-tolyl)octane-1,6,6-tricarboxylate (3na):}

Colorless oil, The crude mixture was purified by column chromatography on silica gel with PE/EA (20:1 to 10:1) as eluent to give $74.8 \mathrm{mg}$, (82\% yield). ${ }^{1} \mathrm{H}$ NMR $\left(600 \mathrm{MHz}, \mathrm{CDCl}_{3}\right) \delta 7.87$ $(\mathrm{d}, J=8.2 \mathrm{~Hz}, 2 \mathrm{H}), 7.27 \quad(\mathrm{~d}, J=8.1 \mathrm{~Hz}, 2 \mathrm{H}), 4.28(\mathrm{q}, J=7.0 \mathrm{~Hz}, 2 \mathrm{H}), 4.22-4.19(\mathrm{~m}, 4 \mathrm{H}), 3.66$ (s, 2H), $2.41(\mathrm{~s}, 3 \mathrm{H}), 2.16(\mathrm{t}, J=8.6 \mathrm{~Hz}, 2 \mathrm{H}), 2.05-2.01(\mathrm{~m}, 2 \mathrm{H}), 1.44-1.41(\mathrm{~m}, 2 \mathrm{H}), 1.29$ (t, $J$ $=7.0 \mathrm{~Hz}, 3 \mathrm{H}), 1.23(\mathrm{t}, J=7.1 \mathrm{~Hz}, 6 \mathrm{H}) ;{ }^{19} \mathrm{~F} \mathrm{NMR}\left(564 \mathrm{MHz}, \mathrm{CDCl}_{3}\right) \delta-106.16(\mathrm{t}, J=17.3 \mathrm{~Hz}, 2 \mathrm{~F})$; ${ }^{13} \mathrm{C}$ NMR $\left(150 \mathrm{MHz}, \mathrm{CDCl}_{3}\right) \delta 196.10,170.62,164.08(\mathrm{t}, J=32.3 \mathrm{~Hz}), 144.35,133.96,129.32$, $128.13,115.78$ (t, $J=248.5 \mathrm{~Hz}), 62.79,61.64,55.18,41.10,34.51$ (t, $J=22.9 \mathrm{~Hz}), 32.39,21.66$, 17.02, 13.95, 13.87; HRMS (ESI) $\mathrm{m} / \mathrm{z}$ calcd for $\mathrm{C}_{23} \mathrm{H}_{31} \mathrm{~F}_{2} \mathrm{O}_{7}\left[\mathrm{M}+\mathrm{H}^{+}\right]$457.2032, found 457.2026. 
<smiles>CCOC(=O)CCCC(CC)(CCCC(=O)c1ccc(OC)cc1)OCC</smiles>

triethyl 1,1-difluoro-8-(4-methoxyphenyl)-8-oxooctane-1,6,6-tricarboxylate (3oa):

Colorless oil, The crude mixture was purified by column chromatography on silica gel with PE/EA (10:1 to 5:1) as eluent to give $81.2 \mathrm{mg}$, (86\% yield). ${ }^{1} \mathrm{H}$ NMR $\left(600 \mathrm{MHz}, \mathrm{CDCl}_{3}\right) \delta 7.95(\mathrm{~d}$, $J=8.9 \mathrm{~Hz}, 2 \mathrm{H}), 6.94(\mathrm{~d}, J=8.8 \mathrm{~Hz}, 2 \mathrm{H}), 4.27$ (q, $J=7.3 \mathrm{~Hz}, 2 \mathrm{H}), 4.22-4.18(\mathrm{~m}, 4 \mathrm{H}), 3.86(\mathrm{~s}$, $3 \mathrm{H}), 3.64(\mathrm{~s}, 3 \mathrm{H}), 2.17(\mathrm{t}, J=8.6 \mathrm{~Hz}, 2 \mathrm{H}), 2.04-2.01(\mathrm{~m}, 2 \mathrm{H}), 1.45-1.39(\mathrm{~m}, 2 \mathrm{H}), 1.29(\mathrm{t}, J=$ $7.0 \mathrm{~Hz}, 3 \mathrm{H}), 1.23(\mathrm{t}, J=6.9 \mathrm{~Hz}, 6 \mathrm{H}) ;{ }^{19} \mathrm{~F} \mathrm{NMR}\left(564 \mathrm{MHz}, \mathrm{CDCl}_{3}\right) \delta-106.16(\mathrm{t}, J=17.3 \mathrm{~Hz}, 2 \mathrm{~F})$; ${ }^{13} \mathrm{C}$ NMR $\left(150 \mathrm{MHz}, \mathrm{CDCl}_{3}\right) \delta 194.94,170.64,164.05(\mathrm{t}, J=32.6 \mathrm{~Hz}), 163.73,130.30,129.51$, $115.77(\mathrm{t}, J=249.0 \mathrm{~Hz}), 113.75,62.76,61.59,55.47,55.19,40.82,34.50(\mathrm{t}, J=23.1 \mathrm{~Hz}), 32.36$, 17.01, 13.92, 13.84; HRMS (ESI) $\mathrm{m} / \mathrm{z}$ calcd for $\mathrm{C}_{23} \mathrm{H}_{31} \mathrm{~F}_{2} \mathrm{O}_{8}\left[\mathrm{M}+\mathrm{H}^{+}\right] 473.1982$, found 473.1972.

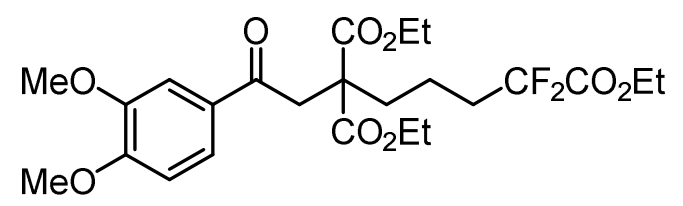

\section{triethyl 8-(3,4-dimethoxyphenyl)-1,1-difluoro-8-oxooctane-1,6,6-tricarboxylate (3pa):}

Colorless oil, The crude mixture was purified by column chromatography on silica gel with PE/EA (10:1 to 5:1) as eluent to give $81.3 \mathrm{mg}$, (81\% yield). ${ }^{1} \mathrm{H}$ NMR $\left(600 \mathrm{MHz}, \mathrm{CDCl}_{3}\right) \delta 7.64$ $\left(\mathrm{dd}, J_{1}=8.3 \mathrm{~Hz}, J_{1}=2.0 \mathrm{~Hz}, 1 \mathrm{H}\right), 7.50(\mathrm{~d}, J=2.0 \mathrm{~Hz}, 1 \mathrm{H}), 6.90(\mathrm{~d}, J=8.6 \mathrm{~Hz}, 1 \mathrm{H}), 4.28(\mathrm{q}, J=$ $7.0 \mathrm{~Hz}, 2 \mathrm{H}), 4.23-4.19$ (m, 4H), 3.95 (s, 3H), $3.92(\mathrm{~s}, 3 \mathrm{H}), 3.66$ (s, 2H), 2.16 (t, $J=8.5 \mathrm{~Hz}, 2 \mathrm{H})$, $2.06-2.01(\mathrm{~m}, 2 \mathrm{H}), 1.46-1.40(\mathrm{~m}, 2 \mathrm{H}), 1.30(\mathrm{t}, J=7.3 \mathrm{~Hz}, 3 \mathrm{H}), 1.24(\mathrm{t}, J=6.9 \mathrm{~Hz}, 6 \mathrm{H}) ;{ }^{19} \mathrm{~F}$ NMR $\left(564 \mathrm{MHz}, \mathrm{CDCl}_{3}\right) \delta-106.21(\mathrm{t}, J=17.3 \mathrm{~Hz}, 2 \mathrm{~F}) ;{ }^{13} \mathrm{C} \mathrm{NMR}\left(150 \mathrm{MHz}, \mathrm{CDCl}_{3}\right) \delta 195.09$, 170.67, 164.08 (t, $J=32.6 \mathrm{~Hz}), 153.58,149.05,129.66,122.82,115.78$ (t, $J=254.4 \mathrm{~Hz}), 109.95$, 109.92, 62.80, 61.65, 56.11, 55.95, 55.27, 40.67, $34.51(\mathrm{t}, J=23.1 \mathrm{~Hz}), 32.35,17.02,13.97,13.88$; HRMS (ESI) $\mathrm{m} / \mathrm{z}$ calcd for $\mathrm{C}_{24} \mathrm{H}_{33} \mathrm{~F}_{2} \mathrm{O}_{9}\left[\mathrm{M}+\mathrm{H}^{+}\right]$503.2078, found 503.2071.

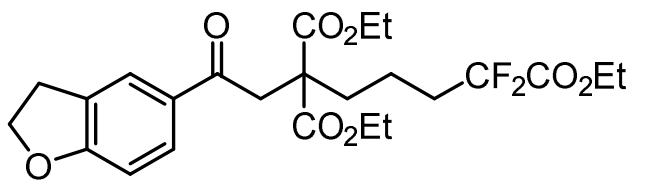

triethyl 7-(2,3-dihydrobenzofuran-5-yl)-1,1-difluoro-7-oxoheptane-1,5,5-tricarboxylate(3qa): Colorless oil, The crude mixture was purified by column chromatography on silica gel with PE/EA (10:1 to 5:1) as eluent to give $78.4 \mathrm{mg}$, (81\% yield). ${ }^{1} \mathrm{H}$ NMR $\left(600 \mathrm{MHz}, \mathrm{CDCl}_{3}\right) \delta 7.84$ (s, $1 \mathrm{H}), 7.82(\mathrm{~d}, J=8.5 \mathrm{~Hz}, 1 \mathrm{H}), 6.81(\mathrm{~d}, J=8.5 \mathrm{~Hz}, 1 \mathrm{H}), 4.66(\mathrm{t}, J=8.8 \mathrm{~Hz}, 2 \mathrm{H}), 4.27$ (q, $J=7.0 \mathrm{~Hz}$, 2H), $4.22-4.18(\mathrm{~m}, 4 \mathrm{H}), 3.62(\mathrm{~s}, 2 \mathrm{H}), 3.25$ (t, $J=8.7 \mathrm{~Hz}, 2 \mathrm{H}), 2.15(\mathrm{t}, J=8.6 \mathrm{~Hz}, 2 \mathrm{H}), 2.06-$ $1.99(\mathrm{~m}, 2 \mathrm{H}), 1.45-1.41(\mathrm{~m}, 2 \mathrm{H}), 1.30(\mathrm{t}, J=7.3 \mathrm{~Hz}, 3 \mathrm{H}), 1.23(\mathrm{t}, J=7.4 \mathrm{~Hz}, 6 \mathrm{H}) ;{ }^{19} \mathrm{~F}$ NMR $\left(564 \mathrm{MHz}, \mathrm{CDCl}_{3}\right) \delta-106.19(\mathrm{t}, J=17.0 \mathrm{~Hz}, 2 \mathrm{~F}) ;{ }^{13} \mathrm{C} \mathrm{NMR}\left(150 \mathrm{MHz}, \mathrm{CDCl}_{3}\right) \delta 194.76,170.69$, 164.65, 164.08 (t, $J=32.4 \mathrm{~Hz}$ ), 130.02, 129.85, 127.76, 125.36, 115.79 (t, $J=248.8 \mathrm{~Hz}), 109.09$, $72.25,62.79,61.59,55.22,40.86,34.52$ (t, $J=23.2 \mathrm{~Hz}), 32.35,28.93,17.00,13.96,13.87$; HRMS (ESI) $\mathrm{m} / \mathrm{z}$ calcd for $\mathrm{C}_{24} \mathrm{H}_{31} \mathrm{~F}_{2} \mathrm{O}_{8}\left[\mathrm{M}+\mathrm{H}^{+}\right]$485.1982, found 485.1978.<smiles>CCOC(=O)CCCCC(CC(=O)c1ccccc1)(OC)C(C)=O</smiles> 
1-ethyl 5,5-dimethyl 1,1-difluoro-7-oxo-7-phenylheptane-1,5,5-tricarboxylate (3ra):

Colorless oil, The crude mixture was purified by column chromatography on silica gel with PE/EA (20:1 to 10:1) as eluent to give $69.5 \mathrm{mg}$, (84\% yield). ${ }^{1} \mathrm{H}$ NMR (600 MHz, $\left.\mathrm{CDCl}_{3}\right) \delta 7.97-$ $7.96(\mathrm{~m}, 2 \mathrm{H}), 7.60-7.57(\mathrm{~m}, 1 \mathrm{H}), 7.49-7.46(\mathrm{~m}, 2 \mathrm{H}), 4.28(\mathrm{t}, J=7.0 \mathrm{~Hz}, 2 \mathrm{H}), 3.74(\mathrm{~s}, 6 \mathrm{H}), 3.70$ (s, 2H), $2.16(\mathrm{t}, J=8.6 \mathrm{~Hz}, 2 \mathrm{H}), 2.06-2.01(\mathrm{~m}, 2 \mathrm{H}), 1.45-1.39(\mathrm{~m}, 2 \mathrm{H}), 1.29(\mathrm{t}, J=7.4 \mathrm{~Hz}, 3 \mathrm{H})$; ${ }^{19} \mathrm{~F}$ NMR $\left(564 \mathrm{MHz}, \mathrm{CDCl}_{3}\right) \delta-106.10(\mathrm{t}, J=17.3 \mathrm{~Hz}, 2 \mathrm{~F}) ;{ }^{13} \mathrm{C} \mathrm{NMR}\left(150 \mathrm{MHz}, \mathrm{CDCl}_{3}\right) \delta$ $196.41,171.04,164.03(\mathrm{t}, J=129.2 \mathrm{~Hz}), 136.24,133.55,128.68,128.02,115.74(\mathrm{t}, J=248.7 \mathrm{~Hz})$, $62.81,55.09,52.84,41.48,34.43(\mathrm{t}, J=23.2 \mathrm{~Hz}$ ), 32.64, 17.07, 13.87; HRMS (ESI) m/z calcd for $\mathrm{C}_{20} \mathrm{H}_{25} \mathrm{~F}_{2} \mathrm{O}_{7}\left[\mathrm{M}+\mathrm{H}^{+}\right]$415.1563, found 415.1551 .<smiles>CCCN(CCC)C(=O)C(F)(F)CCCc1ccccc1C(C)=O</smiles>

5-(2-acetylphenyl)-2,2-difluoro-N,N-dipropylpentanamide (3ab):

Colorless oil, The crude mixture was purified by column chromatography on silica gel with PE/EA (20:1 to $10: 1)$ as eluent to give $48.8 \mathrm{mg}$, (72\% yield). ${ }^{1} \mathrm{H}$ NMR $\left(600 \mathrm{MHz}, \mathrm{CDCl}_{3}\right) \delta 7.67$ $\left(\mathrm{dd}, J_{1}=8.3 \mathrm{~Hz}, J_{2}=1.3 \mathrm{~Hz}, 1 \mathrm{H}\right), 7.41-7.38(\mathrm{~m}, 1 \mathrm{H}), 7.28-7.27(\mathrm{~m}, 2 \mathrm{H}), 3.37(\mathrm{t}, J=7.9 \mathrm{~Hz}$, 2H), $3.26(\mathrm{t}, J=7.7 \mathrm{~Hz}, 2 \mathrm{H}), 2.92(\mathrm{t}, J=7.7 \mathrm{~Hz}, 2 \mathrm{H}), 2.58(\mathrm{~s}, 3 \mathrm{H}), 2.23-2.14(\mathrm{~m}, 2 \mathrm{H}), 1.83-$ $1.81(\mathrm{~m}, 2 \mathrm{H}), 1.60-1.55(\mathrm{~m}, 4 \mathrm{H}), 0.90-0.86(\mathrm{~m}, 6 \mathrm{H}) ;{ }^{19} \mathrm{~F} \mathrm{NMR}\left(564 \mathrm{MHz}, \mathrm{CDCl}_{3}\right) \delta-99.62(\mathrm{t}, J$ $=17.9 \mathrm{~Hz}, 2 \mathrm{~F}) ;{ }^{13} \mathrm{C} \mathrm{NMR}\left(150 \mathrm{MHz}, \mathrm{CDCl}_{3}\right) \delta 201.92,163.20(\mathrm{t}, J=29.3 \mathrm{~Hz}), 141.75,137.69$, $131.51,131.25,129.33,125.99,119.57$ (t, $J=252.5 \mathrm{~Hz}), 119.57,49.15,48.50,34.67$ (t, $J=23.5$ $\mathrm{Hz}$ ), 33.38, 29.79, 23.44, 22.14, 20.23, 11.27, 10.96; HRMS (ESI) m/z calcd for $\mathrm{C}_{19} \mathrm{H}_{28} \mathrm{~F}_{2} \mathrm{NO}_{2}$ $\left[\mathrm{M}+\mathrm{H}^{+}\right]$340.2083, found 340.2071 .<smiles>CC(=O)c1ccccc1CCCC(F)(F)C(=O)N1CCCC1</smiles>

\section{5-(2-acetylphenyl)-2,2-difluoro-1-(pyrrolidin-1-yl)pentan-1-one (3ac):}

Colorless oil, The crude mixture was purified by column chromatography on silica gel with PE/EA (20:1 to 10:1) as eluent to give $40.2 \mathrm{mg},\left(65 \%\right.$ yield). ${ }^{1} \mathrm{H}$ NMR $\left(600 \mathrm{MHz}, \mathrm{CDCl}_{3}\right) \delta 7.65$ $\left(\mathrm{dd}, J_{1}=8.3 \mathrm{~Hz}, J_{2}=1.3 \mathrm{~Hz}, 1 \mathrm{H}\right), 7.39-7.36(\mathrm{~m}, 1 \mathrm{H}), 7.26-7.24(\mathrm{~m}, 2 \mathrm{H}), 3.64(\mathrm{t}, J=6.8 \mathrm{~Hz}$, 2H), $3.48(\mathrm{t}, J=7.0 \mathrm{~Hz}, 2 \mathrm{H}), 2.90(\mathrm{t}, J=7.7 \mathrm{~Hz}, 2 \mathrm{H}), 2.56(\mathrm{~s}, 3 \mathrm{H}), 2.20-2.11(\mathrm{~m}, 2 \mathrm{H}), 1.94-$ $1.89(\mathrm{~m}, 2 \mathrm{H}), 1.84-1.78(\mathrm{~m}, 4 \mathrm{H}) ;{ }^{19} \mathrm{~F}$ NMR $\left(564 \mathrm{MHz}, \mathrm{CDCl}_{3}\right) \delta-103.11(\mathrm{t}, J=17.9 \mathrm{~Hz}, 2 \mathrm{~F}) ;{ }^{13} \mathrm{C}$ NMR $\left(150 \mathrm{MHz}, \mathrm{CDCl}_{3}\right) \delta 201.89,162.28(\mathrm{t}, J=29.7 \mathrm{~Hz}), 141.65,137.61,131.49,131.20$, 129.32, 125.99, 118.99 (t, $J=250.6 \mathrm{~Hz}), 47.28,46.55$ (t, $J=6.1 \mathrm{~Hz}), 33.95$ (t, $J=23.2 \mathrm{~Hz}$ ), 33.31, 29.74, 26.44, 23.32, 23.22; HRMS (ESI) $\mathrm{m} / \mathrm{z}$ calcd for $\mathrm{C}_{17} \mathrm{H}_{22} \mathrm{~F}_{2} \mathrm{NO}_{2}\left[\mathrm{M}+\mathrm{H}^{+}\right] 310.1613$, found 310.1606.<smiles>CC(=O)c1ccccc1CCCC(F)(F)C(=O)N1CCOCC1</smiles> 
5-(2-acetylphenyl)-2,2-difluoro-1-morpholinopentan-1-one (3ad):

Colorless oil, The crude mixture was purified by column chromatography on silica gel with PE/EA (20:1 to 10:1) as eluent to give $52.7 \mathrm{mg}$, (81\% yield). ${ }^{1} \mathrm{H}$ NMR (600 MHz, $\left.\mathrm{CDCl}_{3}\right) \delta 7.69$ $(\mathrm{d}, J=7.5 \mathrm{~Hz}, 1 \mathrm{H}), 7.42-7.39(\mathrm{~m}, 1 \mathrm{H}), 7.30-7.27(\mathrm{~m}, 2 \mathrm{H}), 3.73-3.62(\mathrm{~m}, 8 \mathrm{H}), 2.92(\mathrm{t}, J=8.2$ $\mathrm{Hz}, 2 \mathrm{H}), 2.59(\mathrm{~s}, 3 \mathrm{H}), 2.21-2.18(\mathrm{~m}, 2 \mathrm{H}), 1.86-1.81(\mathrm{~m}, 2 \mathrm{H}) ;{ }^{19} \mathrm{~F}$ NMR $\left(564 \mathrm{MHz}, \mathrm{CDCl}_{3}\right) \delta$ -99.30 (t, $J=17.7 \mathrm{~Hz}, 2 \mathrm{~F}) ;{ }^{13} \mathrm{C}$ NMR $\left(150 \mathrm{MHz}, \mathrm{CDCl}_{3}\right) \delta 201.87,162.07$ (t, $\left.J=28.8 \mathrm{~Hz}\right), 141.70$, $137.60,131.58,131.25,129.45,126.08,119.41(\mathrm{t}, J=252.0 \mathrm{~Hz}), 66.79,66.73,46.51(\mathrm{t}, J=5.7$ $\mathrm{Hz}$ ), 43.28, 34.35 (t, $J=22.9 \mathrm{~Hz}$ ), 33.41, 29.78, 23.37; HRMS (ESI) $\mathrm{m} / \mathrm{z}$ calcd for $\mathrm{C}_{17} \mathrm{H}_{22} \mathrm{~F}_{2} \mathrm{NO}_{3}$ $\left[\mathrm{M}+\mathrm{H}^{+}\right]$326.1562, found 326.1554 .<smiles>CC(=O)c1ccccc1CCCC(F)(F)C(=O)N1CCN(C(=O)OCc2ccccc2)CC1</smiles>

tert-butyl 4-(5-(2-acetylphenyl)-2,2-difluoropentanoyl)piperazine-1-carboxylate (3ae):

Colorless oil, The crude mixture was purified by column chromatography on silica gel with PE/EA (10:1 to 5:1) as eluent to give $65.3 \mathrm{mg}$, (77\% yield). ${ }^{1} \mathrm{H}$ NMR $\left(600 \mathrm{MHz}, \mathrm{CDCl}_{3}\right) \delta 7.65(\mathrm{~d}$, $J=7.6 \mathrm{~Hz}, 1 \mathrm{H}), 7.38-7.36(\mathrm{~m}, 1 \mathrm{H}), 7.25-7.23(\mathrm{~m}, 2 \mathrm{H}), 3.64-3.56(\mathrm{~m}, 4 \mathrm{H}), 3.42-3.40(\mathrm{~m}$, 4H), $2.89(\mathrm{t}, J=7.7 \mathrm{~Hz}, 2 \mathrm{H}), 2.55(\mathrm{~s}, 3 \mathrm{H}), 2.18-2.15(\mathrm{~m}, 2 \mathrm{H}), 1.81-1.79(\mathrm{~m}, 2 \mathrm{H}), 1.43(\mathrm{~s}, 9 \mathrm{H})$; ${ }^{19} \mathrm{~F}$ NMR $\left(564 \mathrm{MHz}, \mathrm{CDCl}_{3}\right) \delta-99.16(\mathrm{~s}, 2 \mathrm{~F}) ;{ }^{13} \mathrm{C} \mathrm{NMR}\left(150 \mathrm{MHz}, \mathrm{CDCl}_{3}\right) \delta 201.81,162.14(\mathrm{t}, J$ $=28.5 \mathrm{~Hz}), 154.42,141.66,137.57,131.54,131.22,129.42,126.05,119.37(\mathrm{t}, J=252.2 \mathrm{~Hz})$, 80.35, 45.65, 42.87, 34.33 (t, $J=22.8 \mathrm{~Hz}$ ), 33.36, 29.73, 28.30, 23.34; HRMS (ESI) $\mathrm{m} / \mathrm{z}$ calcd for $\mathrm{C}_{22} \mathrm{H}_{31} \mathrm{~F}_{2} \mathrm{~N}_{2} \mathrm{O}_{4}\left[\mathrm{M}+\mathrm{H}^{+}\right]$425.2246, found 425.2253.

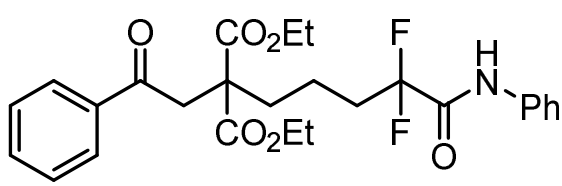

diethyl 2-(4,4-difluoro-5-oxo-5-(phenylamino)pentyl)-2-(2-oxo-2-phenylethyl)malonate (3af): White solid, The crude mixture was purified by column chromatography on silica gel with PE/EA (10:1 to 5:1) as eluent to give $68.5 \mathrm{mg},\left(70 \%\right.$ yield). ${ }^{1} \mathrm{H}$ NMR $\left(600 \mathrm{MHz}, \mathrm{CDCl}_{3}\right) \delta 8.02(\mathrm{br}, 1 \mathrm{H})$, $7.96\left(\mathrm{dd}, J_{1}=8.0 \mathrm{~Hz}, J_{2}=1.1 \mathrm{~Hz}, 2 \mathrm{H}\right), 7.58-7.53(\mathrm{~m}, 3 \mathrm{H}), 7.45(\mathrm{t}, J=7.9 \mathrm{~Hz}, 2 \mathrm{H}), 7.34(\mathrm{t}, \mathrm{J}=$ $7.6 \mathrm{~Hz}, 2 \mathrm{H}), 7.19-7.16(\mathrm{~m}, 1 \mathrm{H}), 4.21$ (q, $J=7.1 \mathrm{~Hz}, 4 \mathrm{H}), 3.69$ (s, 2H), $2.21-2.13$ (m, 4H), 1.52 $-1.47(\mathrm{~m}, 2 \mathrm{H}), 1.21(\mathrm{t}, J=7.0 \mathrm{~Hz}, 6 \mathrm{H}) ;{ }^{19} \mathrm{~F}$ NMR $\left(564 \mathrm{MHz}, \mathrm{CDCl}_{3}\right) \delta-105.37(\mathrm{t}, J=17.2 \mathrm{~Hz}$, $2 \mathrm{~F}) ;{ }^{13} \mathrm{C}$ NMR $\left(150 \mathrm{MHz}, \mathrm{CDCl}_{3}\right) \delta 195.55,170.60,161.84(\mathrm{t}, J=28.3 \mathrm{~Hz}), 136.41,135.93$, 133.42, 129.14, 128.63, 128.00, 125.52, 120.17, 117.91 (t, $J=252.3 \mathrm{~Hz}), 61.68,55.21,41.35$, $33.74\left(\mathrm{t}, J=22.8 \mathrm{~Hz}\right.$ ), 32.53, 17.23, 13.93; HRMS (ESI) $\mathrm{m} / \mathrm{z}$ calcd for $\mathrm{C}_{26} \mathrm{H}_{30} \mathrm{~F}_{2} \mathrm{NO}_{6}\left[\mathrm{M}+\mathrm{H}^{+}\right]$ 490.2036, found 490.2031 .

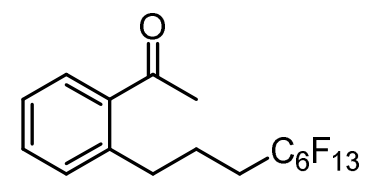

1-(2-(4,4,5,5,6,6,7,7,8,8,9,9,9-tridecafluorononyl)phenyl)ethan-1-one (3ag):

Colorless oil, The crude mixture was purified by column chromatography on silica gel with 
PE/EA (50:1 to 20:1) as eluent to give $66.2 \mathrm{mg},\left(69 \%\right.$ yield). ${ }^{1} \mathrm{H}$ NMR $\left(600 \mathrm{MHz}, \mathrm{CDCl}_{3}\right) \delta 7.74$ $\left(\mathrm{dd}, J_{1}=7.6 \mathrm{~Hz}, J_{2}=0.8 \mathrm{~Hz}, 1 \mathrm{H}\right), 7.45\left(\mathrm{dt}, J_{1}=7.5 \mathrm{~Hz}, J_{2}=1.1 \mathrm{~Hz}, 1 \mathrm{H}\right), 7.33\left(\mathrm{dt}, J_{1}=7.6 \mathrm{~Hz}, J_{2}\right.$ $=1.0 \mathrm{~Hz}, 1 \mathrm{H}), 7.27(\mathrm{~s}, 1 \mathrm{H}), 2.94(\mathrm{t}, J=7.8 \mathrm{~Hz}, 2 \mathrm{H}), 2.60(\mathrm{~s}, 3 \mathrm{H}), 2.18-2.10(\mathrm{~m}, 2 \mathrm{H}), 1.93-1.88$ $(\mathrm{m}, 2 \mathrm{H}) ;{ }^{19} \mathrm{~F}$ NMR $\left(564 \mathrm{MHz}, \mathrm{CDCl}_{3}\right) \delta-80.68(\mathrm{t}, J=10.0 \mathrm{~Hz}, 3 \mathrm{~F}),-114.17(\mathrm{t}, J=15.5 \mathrm{~Hz}, 2 \mathrm{~F})$, $-121.88(\mathrm{~s}, 2 \mathrm{~F}),-122.82(\mathrm{~s}, 2 \mathrm{~F}),-123.43$ (s, 2F), -126.07 (s, 2F); $\left.{ }^{13} \mathrm{C} \mathrm{NMR} \mathrm{(150} \mathrm{MHz,} \mathrm{CDCl}_{3}\right) \delta$ 201.55, 141.41, 137.22, 131.84, 131.30, 129.85, 126.37, 33.36, 30.64 (t, $J=21.9 \mathrm{~Hz}$ ), 29.62, 22.18; HRMS (ESI) $\mathrm{m} / \mathrm{z}$ calcd for $\mathrm{C}_{17} \mathrm{H}_{14} \mathrm{~F}_{13} \mathrm{O}\left[\mathrm{M}+\mathrm{H}^{+}\right]$481.0832, found 481.0830 .<smiles>CCOC(=O)C(CCCC(F)(F)F)(CC(=O)c1ccccc1)C(=O)OCC</smiles>

diethyl 2-(2-0xo-2-phenylethyl)-2-(4,4,5,5,6,6,7,7,8,8,9,9,9-tridecafluorononyl)malonate(3gg): Colorless oil, The crude mixture was purified by column chromatography on silica gel with PE/EA (50:1 to 20:1) as eluent to give $82.9 \mathrm{mg},\left(65 \%\right.$ yield). ${ }^{1} \mathrm{H}$ NMR $\left(600 \mathrm{MHz}, \mathrm{CDCl}_{3}\right) \delta 7.99$ $\left(\mathrm{dd}, J_{1}=8.0 \mathrm{~Hz}, J_{2}=1.1 \mathrm{~Hz}, 2 \mathrm{H}\right), 7.61-7.58(\mathrm{~m}, 1 \mathrm{H}), 7.48(\mathrm{t}, J=7.9 \mathrm{~Hz}, 2 \mathrm{H}), 4.24\left(\mathrm{dq}, J_{1}=7.1\right.$ $\left.\mathrm{Hz}, J_{2}=1.3 \mathrm{~Hz}, 4 \mathrm{H}\right), 3.72(\mathrm{~s}, 2 \mathrm{H}), 2.21(\mathrm{t}, J=5.4 \mathrm{~Hz}, 2 \mathrm{H}), 2.19-2.03(\mathrm{~m}, 2 \mathrm{H}), 1.60-1.56(\mathrm{~m}$, 2H), $1.24(\mathrm{t}, J=7.3 \mathrm{~Hz}, 6 \mathrm{H}) ;{ }^{19} \mathrm{~F} \mathrm{NMR}\left(564 \mathrm{MHz}, \mathrm{CDCl}_{3}\right) \delta-80.68(\mathrm{t}, J=8.7 \mathrm{~Hz}, 3 \mathrm{~F}),-114.26(\mathrm{t}$, $J=15.0 \mathrm{~Hz}, 2 \mathrm{~F}),-121.89(\mathrm{~s}, 2 \mathrm{~F}),-122.82(\mathrm{~s}, 2 \mathrm{~F}),-123.53(\mathrm{~s}, 2 \mathrm{~F}),-126.07$ (s, 2F); ${ }^{13} \mathrm{C}$ NMR $(150$ $\left.\mathrm{MHz}, \mathrm{CDCl}_{3}\right) \delta 196.48,170.50,136.39,133.53,128.69,128.02,61.76,55.18,41.44,32.46,31.00$ (t, $J=21.8 \mathrm{~Hz}$ ), 15.97, 13.95; HRMS (ESI) $\mathrm{m} / \mathrm{z}$ calcd for $\mathrm{C}_{24} \mathrm{H}_{24} \mathrm{~F}_{13} \mathrm{O}_{5}\left[\mathrm{M}+\mathrm{H}^{+}\right] 639.1411$, found 639.1401 .<smiles>CCOC(=O)C(CCCC(F)F)(CC(=O)c1ccccc1)C(=O)OCC</smiles>

Diethyl 2-(4,4,5,5,6,6,7,7,8,8,9,9,10,10,11,11,11-heptadecafluoroundecyl)-2-(2-oxo-2-phenyl -ethyl) malonate (3gh):

Colorless oil, The crude mixture was purified by column chromatography on silica gel with PE/EA (50:1 to 20:1) as eluent to give $97.4 \mathrm{mg}$, (66\% yield). ${ }^{1} \mathrm{H}$ NMR $\left(600 \mathrm{MHz}, \mathrm{CDCl}_{3}\right) \delta 7.98$ $\left(\mathrm{dd}, J_{1}=8.1 \mathrm{~Hz}, J_{2}=1.0 \mathrm{~Hz}, 2 \mathrm{H}\right), 7.61-7.58(\mathrm{~m}, 1 \mathrm{H}), 7.48(\mathrm{t}, J=7.8 \mathrm{~Hz}, 2 \mathrm{H}), 4.24\left(\mathrm{dq}, J_{1}=7.0\right.$ $\left.\mathrm{Hz}, J_{2}=1.4 \mathrm{~Hz}, 4 \mathrm{H}\right), 3.72(\mathrm{~s}, 2 \mathrm{H}), 2.20(\mathrm{t}, J=8.4 \mathrm{~Hz}, 2 \mathrm{H}), 2.10-2.02(\mathrm{~m}, 2 \mathrm{H}), 1.57-1.54(\mathrm{~m}$, 2H), $1.24(\mathrm{t}, J=7.3 \mathrm{~Hz}, 6 \mathrm{H}) ;{ }^{19} \mathrm{~F}$ NMR $\left(564 \mathrm{MHz}, \mathrm{CDCl}_{3}\right) \delta-80.63(\mathrm{t}, J=8.5 \mathrm{~Hz}, 3 \mathrm{~F}),-114.23(\mathrm{t}$, $J=15.2 \mathrm{~Hz}, 2 \mathrm{~F}),-121.66--121.85(\mathrm{~m}, 8 \mathrm{~F}),-122.63(\mathrm{~s}, 2 \mathrm{~F}),-123.47(\mathrm{~s}, 2 \mathrm{~F}),-126.0(\mathrm{~s}, 2 \mathrm{~F}) ;{ }^{13} \mathrm{C}$ NMR $\left(150 \mathrm{MHz}, \mathrm{CDCl}_{3}\right) \delta 196.49,170.50,136.39,133.53,128.70,128.02,61.76,55.18,41.43$, $32.46,31.01(\mathrm{t}, J=22.5 \mathrm{~Hz}), 15.97,13.95$; HRMS (ESI) $\mathrm{m} / \mathrm{z}$ calcd for $\mathrm{C}_{26} \mathrm{H}_{24} \mathrm{~F}_{17} \mathrm{O}_{5}\left[\mathrm{M}+\mathrm{H}^{+}\right]$ 739.1347 , found 739.1350 .<smiles>CCOC(=O)C(F)(CCCC(OCC)(C(=O)OC)C(=O)OCC)CC(=O)c1ccccc1</smiles>

\section{5,5-diethyl 1-methyl 1,1-difluoro-7-oxo-7-phenylheptane-1,5,5-tricarboxylate (3gi):}

Colorless oil, The crude mixture was purified by column chromatography on silica gel with PE/EA (20:1 to 10:1) as eluent to give $71.9 \mathrm{mg}$, (84\% yield). ${ }^{1} \mathrm{H}$ NMR (600 MHz, $\left.\mathrm{CDCl}_{3}\right) \delta 7.98$ $\left(\mathrm{dd}, J_{1}=7.7 \mathrm{~Hz}, J_{2}=6.1 \mathrm{~Hz}, 2 \mathrm{H}\right), 7.60-7.57(\mathrm{~m}, 1 \mathrm{H}), 7.49-7.46(\mathrm{~m}, 2 \mathrm{H}), 4.23\left(\mathrm{dq}, J_{1}=6.9 \mathrm{~Hz}\right.$, 
$\left.J_{2}=2.0 \mathrm{~Hz}, 4 \mathrm{H}\right), 3.81(\mathrm{~s}, 3 \mathrm{H}), 3.69(\mathrm{~s}, 2 \mathrm{H}), 2.16(\mathrm{t}, J=8.6 \mathrm{~Hz}, 2 \mathrm{H}), 2.09-2.00(\mathrm{~m}, 2 \mathrm{H}), 1.46-$ $1.41(\mathrm{~m}, 2 \mathrm{H}), 1.23(\mathrm{t}, J=7.0 \mathrm{~Hz}, 6 \mathrm{H}) ;{ }^{19} \mathrm{~F} \mathrm{NMR}\left(564 \mathrm{MHz}, \mathrm{CDCl}_{3}\right) \delta-105.87(\mathrm{t}, J=17.3 \mathrm{~Hz}, 2 \mathrm{~F})$; ${ }^{13} \mathrm{C} \mathrm{NMR}\left(150 \mathrm{MHz}, \mathrm{CDCl}_{3}\right) \delta 196.51,170.54,164.54(\mathrm{t}, J=33.1 \mathrm{~Hz}), 136.43,133.46,128.67$, 128.01, 115.82 (t, $J=248.6 \mathrm{~Hz}), 61.68,55.20,53.27,41.28,34.52(\mathrm{t}, J=23.2 \mathrm{~Hz}), 32.41,17.02$, 13.95; HRMS (ESI) $\mathrm{m} / \mathrm{z}$ calcd for $\mathrm{C}_{21} \mathrm{H}_{27} \mathrm{~F}_{2} \mathrm{O}_{7}\left[\mathrm{M}+\mathrm{H}^{+}\right]$429.1719, found 429.1710 .

\section{Control and isotope labeling experiments}<smiles>C=CCc1ccccc1CC</smiles>

1a<smiles>CCOC(=O)C(F)(F)Br</smiles>

$2 a$<smiles>CCOC(=O)OCc1ccccc1C(C)=O</smiles>

3a, ND<smiles>CCOC(=O)C(F)(F)ON1C(C)(C)CCCC1(C)C</smiles>

detected by HRMS 280.1739

To an oven-dried $10 \mathrm{~mL}$ Schlenk tube was added $f a c-\operatorname{Ir}(\mathrm{ppy})_{3}(2 \mathrm{~mol} \%)$ and $\mathrm{AgBz}(0.3 \mathrm{mmol})$. The tube was evacuated and back-filled with nitrogen (this process was repeated three times), alkene $(0.2 \mathrm{mmol})$, alkyl bromide $(0.3 \mathrm{mmol})$, Tempo $(1.2 \mathrm{mmol})$ and DMSO $(2.0 \mathrm{~mL})$ was added subsequently via syring. The resulting mixture was stirred at room temperature with $12 \mathrm{~W}$ blue LED irradiation for 24 hours. The mixture was determined by HRMS. HRMS (ESI) $\mathrm{m} / \mathrm{z}$ calcd for $\mathrm{C}_{13} \mathrm{H}_{24} \mathrm{~F}_{2} \mathrm{NO}_{3}\left[\mathrm{M}+\mathrm{H}^{+}\right]$280.1723, found 280.1739 .

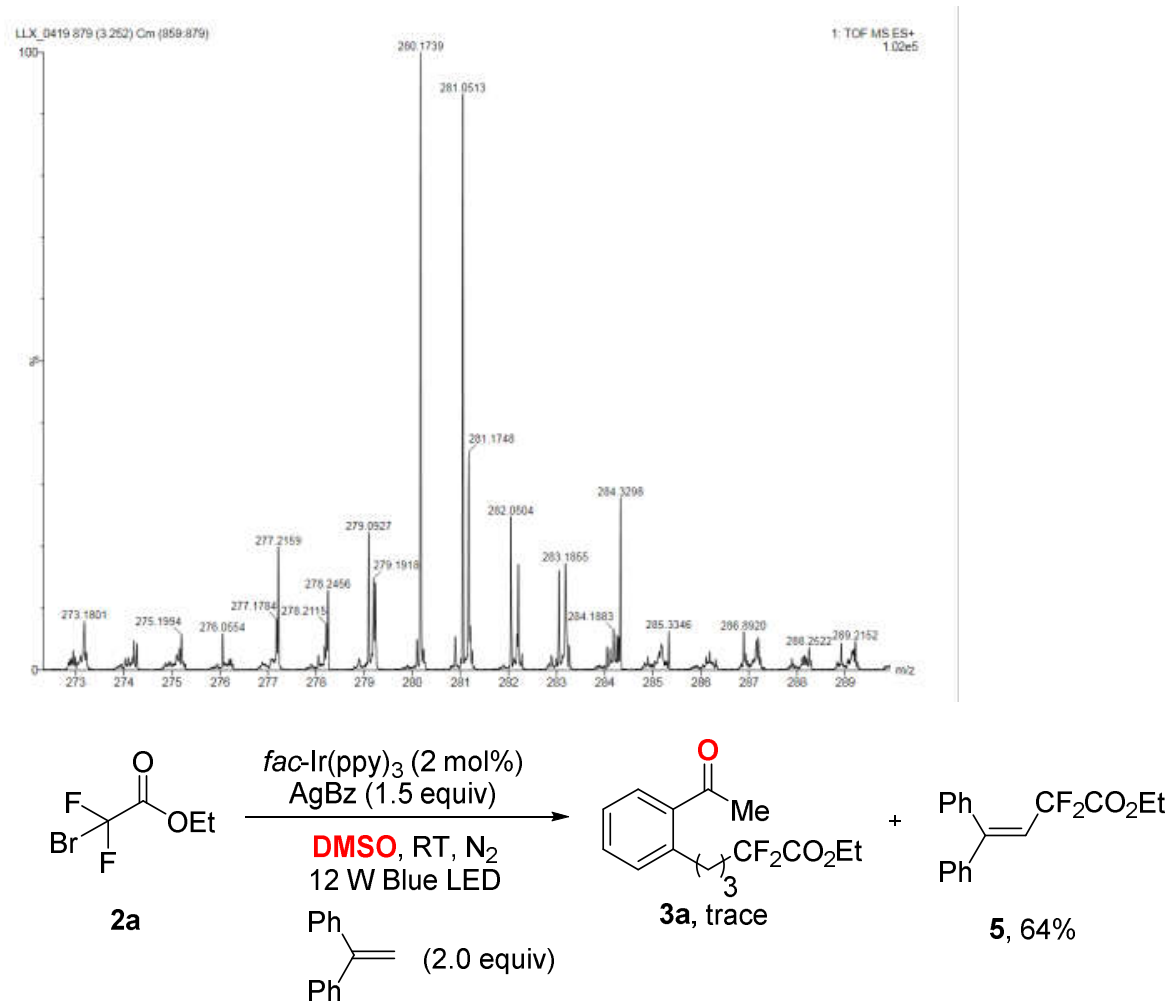

To an oven-dried $10 \mathrm{~mL}$ Schlenk tube was added $f a c-\operatorname{Ir}(\mathrm{ppy})_{3}(2 \mathrm{~mol} \%)$ and $\mathrm{AgBz}(0.3 \mathrm{mmol})$. The tube was evacuated and back-filled with nitrogen (this process was repeated three times). 1a $(0.2 \mathrm{mmol}), 2 \mathrm{a}(0.3 \mathrm{mmol})$, ethene-1,1-diyldibenzene $(0.4 \mathrm{mmol})$ and DMSO $(2.0 \mathrm{~mL})$ was added subsequently via syring. The resulting mixture was stirred at room temperature with $12 \mathrm{~W}$ blue LED irradiation for 24 hours. Then the mixture was diluted with EA $(2.0 \mathrm{~mL} \times 3)$. The organic was washed with saturated $\mathrm{NaCl}$ solution, dired over anhydrous $\mathrm{Na}_{2} \mathrm{SO}_{4}$, filtered and concentrated 
under reduced pressure. The crude mixture was purified by column chromatography on silica gel with PE/EA mixture $(50: 1$ to $20: 1)$ as eluent to give the titled products in pure form.<smiles>CCOC(=O)C(F)(F)F</smiles>

\section{ethyl 2,2-difluoro-4,4-diphenylbut-3-enoate (5):}

Colorless oil, $57.9 \mathrm{mg}$, (64\% yield). ${ }^{1} \mathrm{H}$ NMR (600 MHz, $\left.\mathrm{CDCl}_{3}\right) \delta 7.37-7.36(\mathrm{~m}, 6 \mathrm{H}), 7.33-7.31$ (m, 2H), $7.25-7.19(\mathrm{~m}, 2 \mathrm{H}), 6.26(\mathrm{t}, J=11.9 \mathrm{~Hz}, 1 \mathrm{H}), 3.91(\mathrm{q}, J=7.2 \mathrm{~Hz}, 2 \mathrm{H}), 1.17$ (t, $J=7.2$ $\mathrm{Hz}, 3 \mathrm{H}) ;{ }^{19} \mathrm{~F}$ NMR $\left(564 \mathrm{MHz}, \mathrm{CDCl}_{3}\right) \delta-90.78(\mathrm{~d}, J=12.1 \mathrm{~Hz}, 2 \mathrm{~F}) ;{ }^{13} \mathrm{C} \mathrm{NMR}\left(150 \mathrm{MHz}, \mathrm{CDCl}_{3}\right)$ $\delta 163.42(\mathrm{t}, J=33.3 \mathrm{~Hz}), 150.95(\mathrm{t}, J=37.0 \mathrm{~Hz}), 140.42,137.02,128.36,127.97,127.87,119.45$ (t, $J=28.3 \mathrm{~Hz}), 112.51(\mathrm{t}, J=243.7 \mathrm{~Hz}), 62.73,13.65$.

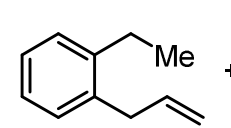

1a<smiles>CCOC(=O)C(F)(F)Br</smiles>

2a<smiles>CCOC(=O)CCCc1ccccc1C(C)=O</smiles>

$3 a^{\prime}$

To an oven-dried $10 \mathrm{~mL}$ Schlenk tube was added $f a c-\operatorname{Ir}(\mathrm{ppy})_{3}(2 \mathrm{~mol} \%)$ and $\mathrm{AgBz}(0.15 \mathrm{mmol})$. The tube was evacuated and back-filled with nitrogen (this process was repeated three times). 1a $(0.1 \mathrm{mmol}), \mathbf{2 a}(0.15 \mathrm{mmol})$ and DMSO-18 $(1.0 \mathrm{~mL})$ was added subsequently via syring. The resulting mixture was stirred at room temperature with $12 \mathrm{~W}$ blue LED irradiating for 24 hours. Then the mixture was treated with $\mathrm{EA}(1.0 \mathrm{~mL} \times 3)$ and diluted by saturated $\mathrm{NaCl}$ solution, dried over anhydrous $\mathrm{Na}_{2} \mathrm{SO}_{4}$, filtered and concentrated under reduced pressure. The crude mixture was purified by column chromatography on silica gel with PE/EA (50:1 to 20:1) as eluent to give the desired product 3a' (19.2 mg, 67\% yield). HRMS (ESI) $\mathrm{m} / \mathrm{z}$ calcd for $\mathrm{C}_{15} \mathrm{H}_{19} \mathrm{~F}_{2} \mathrm{O}_{2}{ }^{18} \mathrm{O}\left[\mathrm{M}+\mathrm{H}^{+}\right]$ 287.1339, found 287.1347.

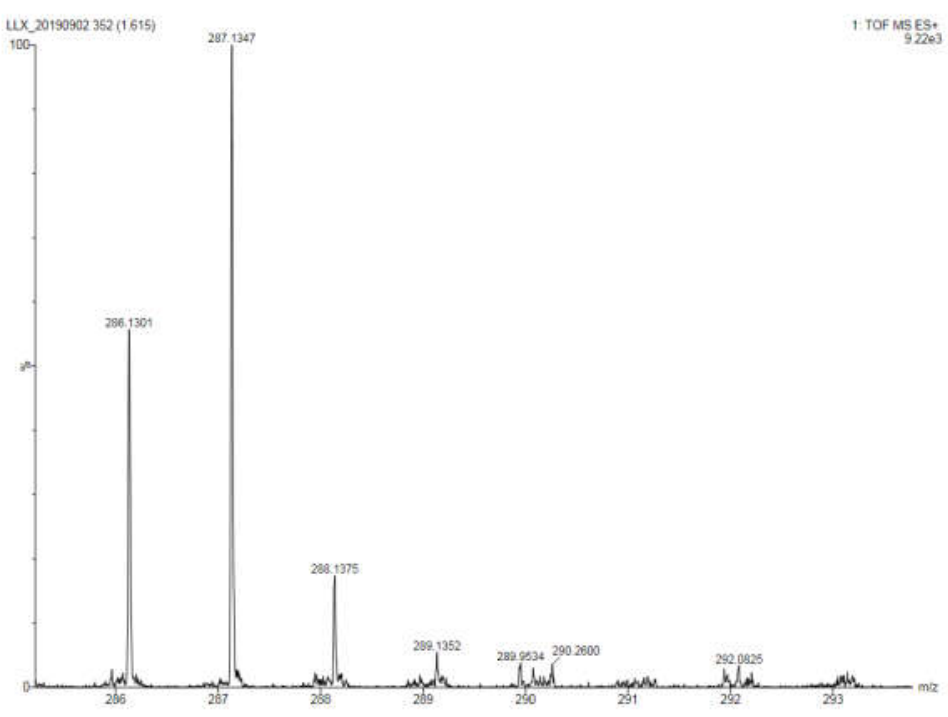


6. Spectra of new compounds

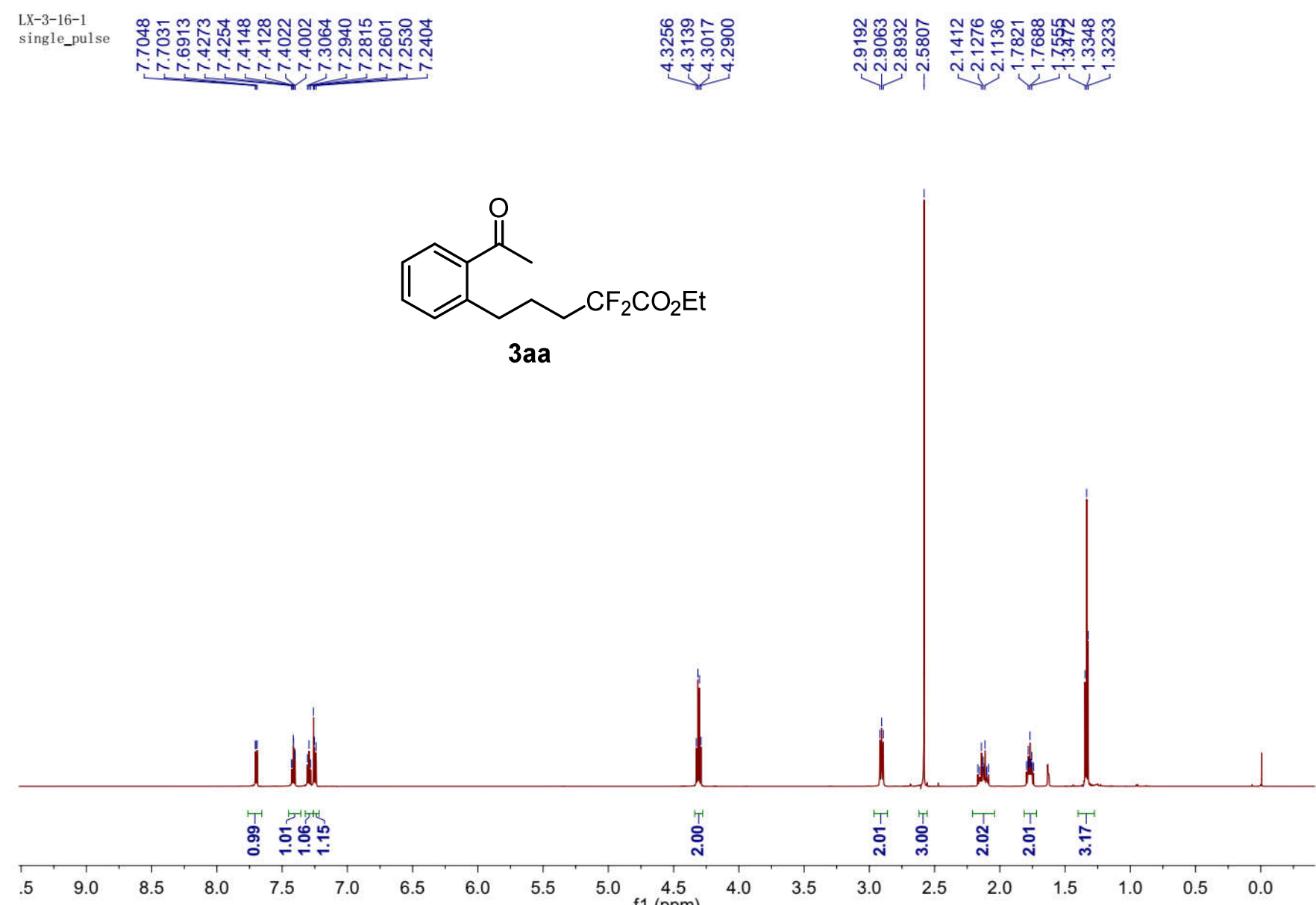


LX-3-16-1

single_puls

:잉

응ㅎํㅇํㅇ

군
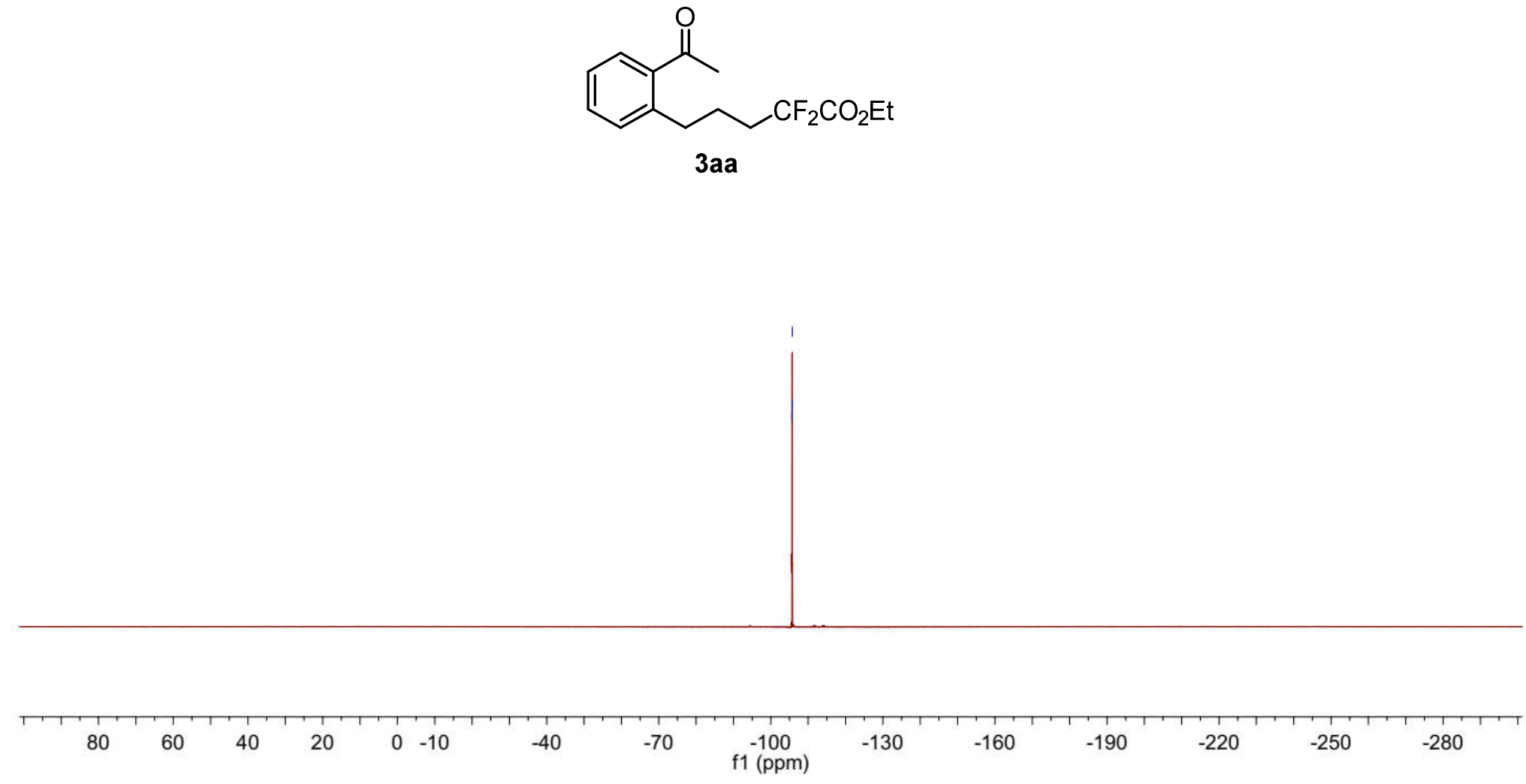


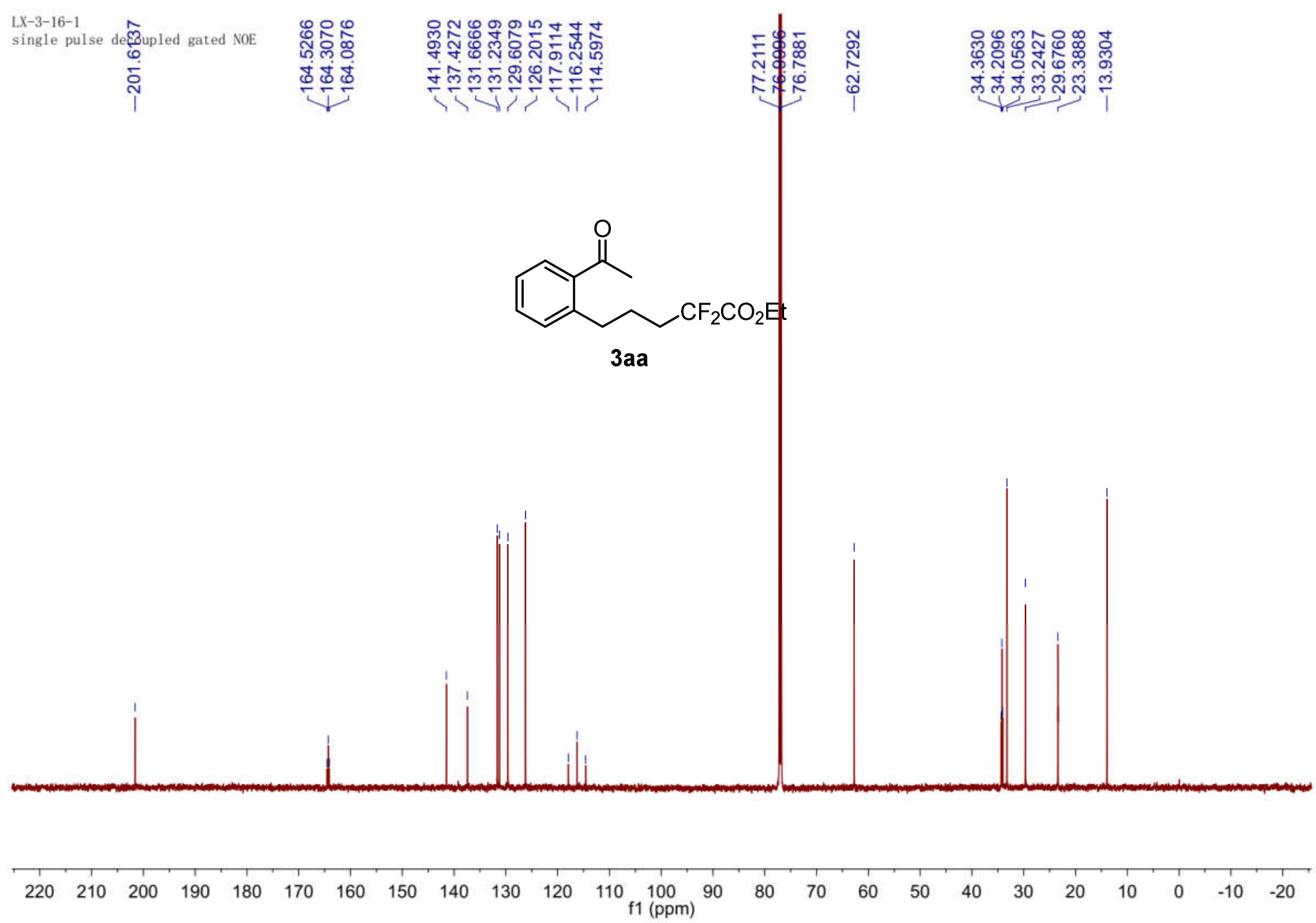




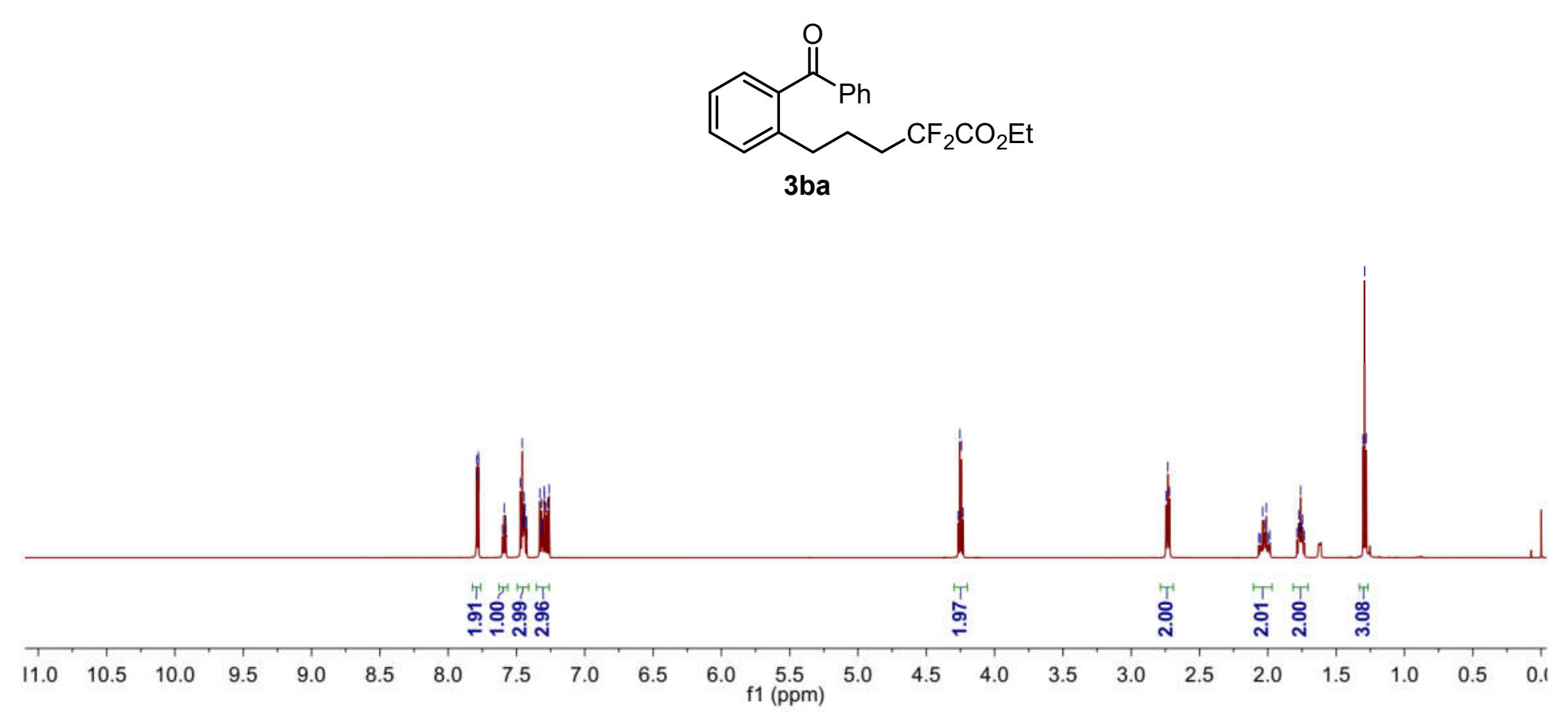


LX-3-26-2

LX-3-26-2
single_pulse

हू.

किष

눈

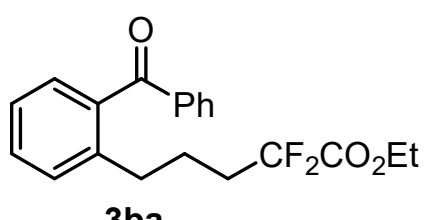

3 ba

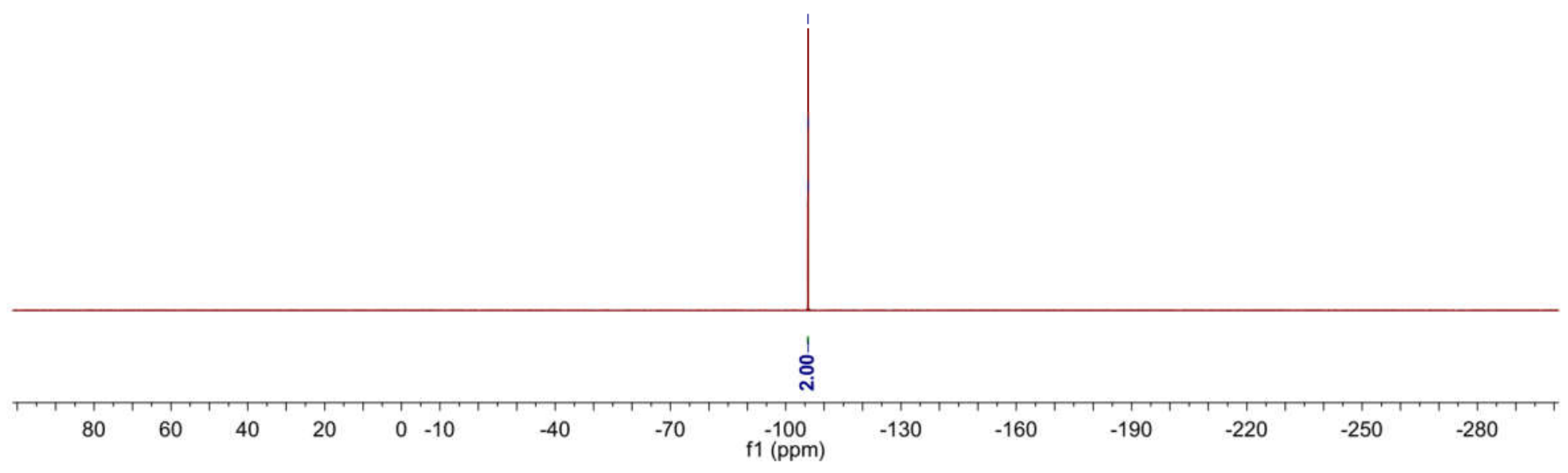




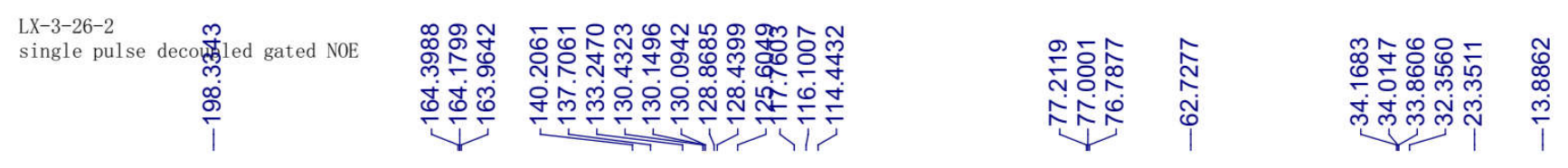
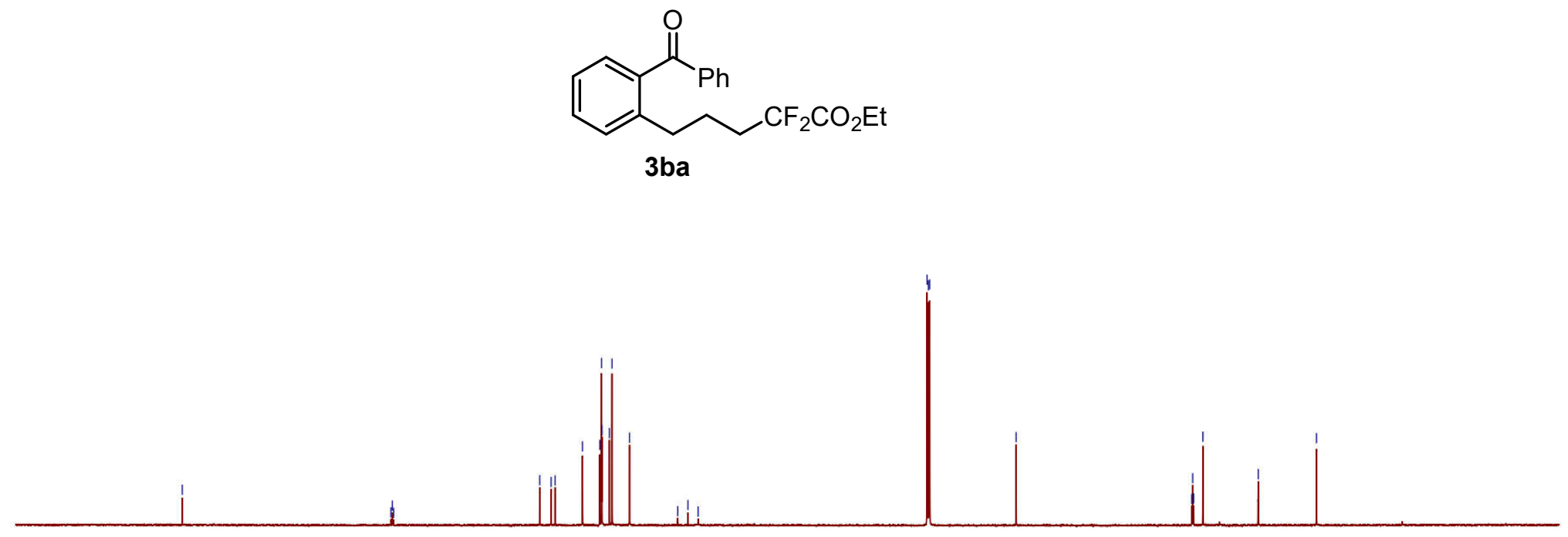

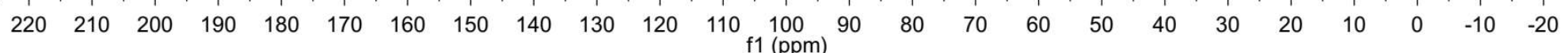




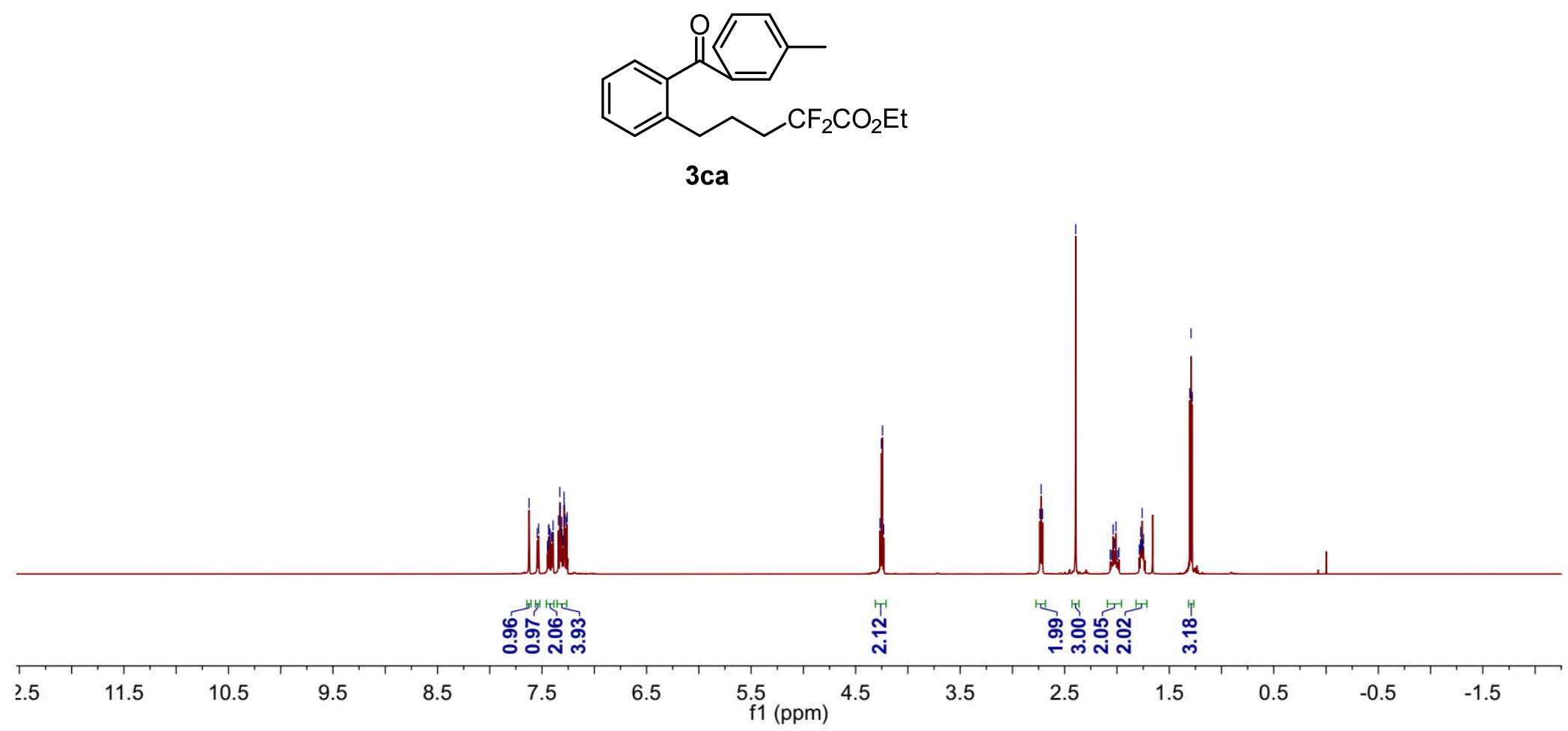


LX-3-41
single_pulse

:

ํํㄴ
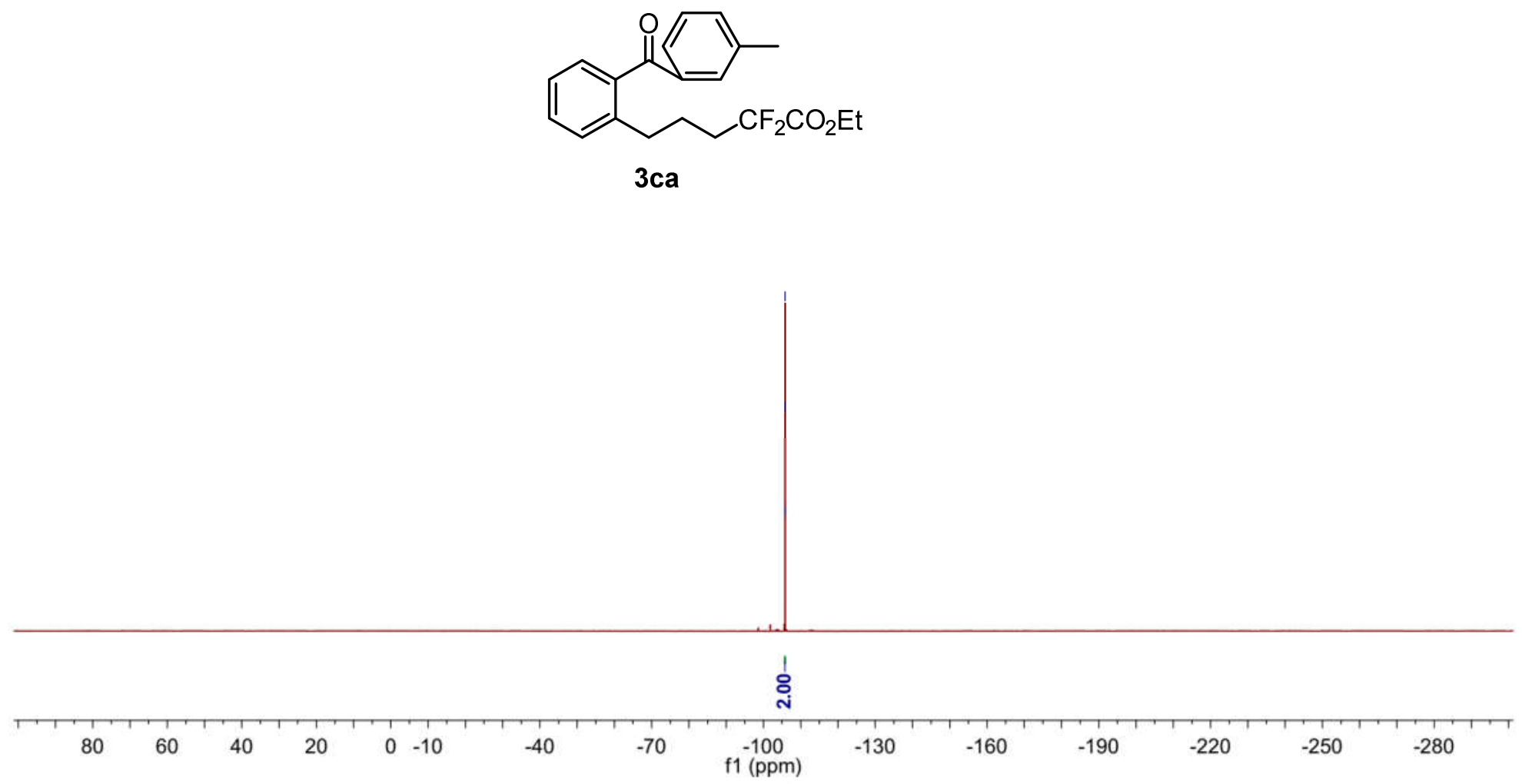


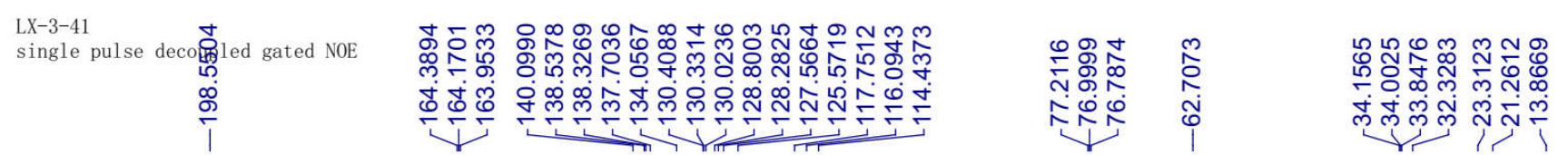

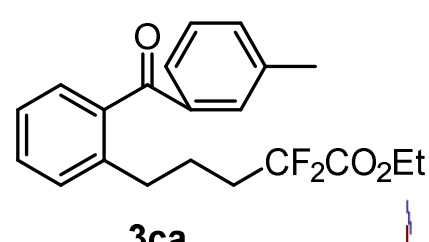

$3 \mathrm{ca}$

$\begin{array}{lllllllllllllllllllllllll}220 & 210 & 200 & 190 & 180 & 170 & 160 & 150 & 140 & 130 & 120 & 110 & \begin{array}{c}100 \\ \mathrm{f} 1(\mathrm{ppm})\end{array} & 90 & 80 & 70 & 60 & 50 & 40 & 30 & 20 & 10 & 0 & -10 & -20\end{array}$ 


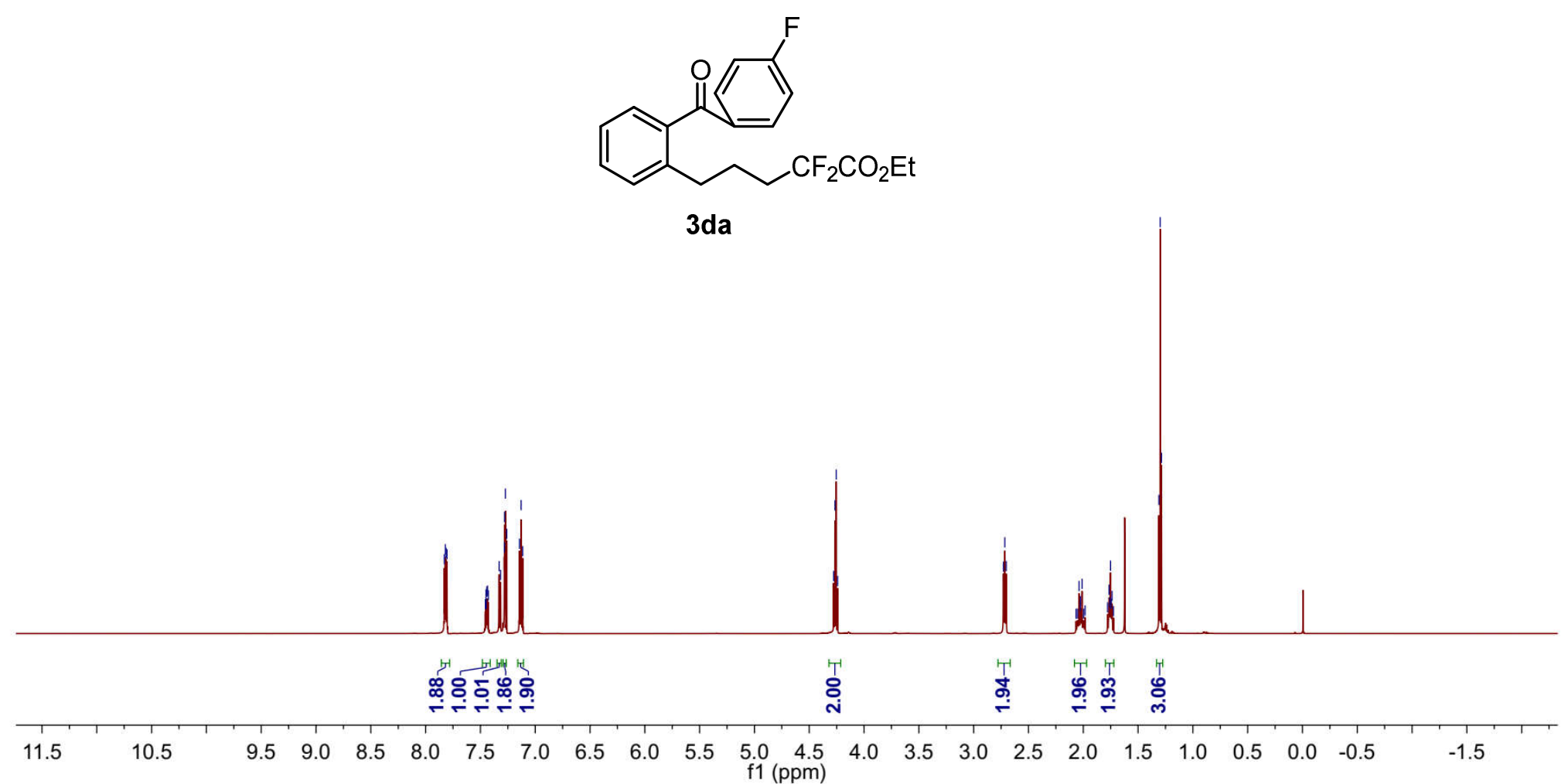



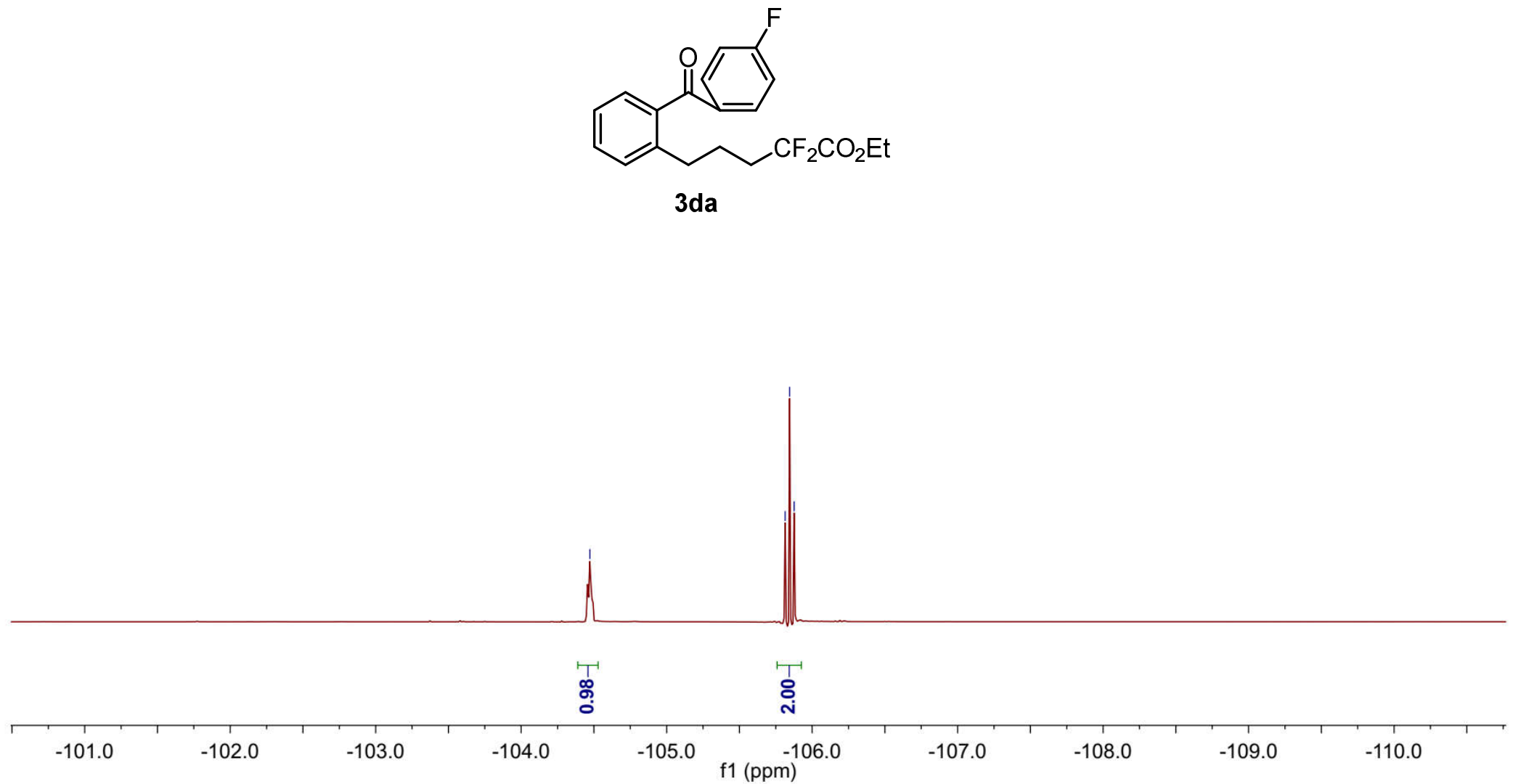


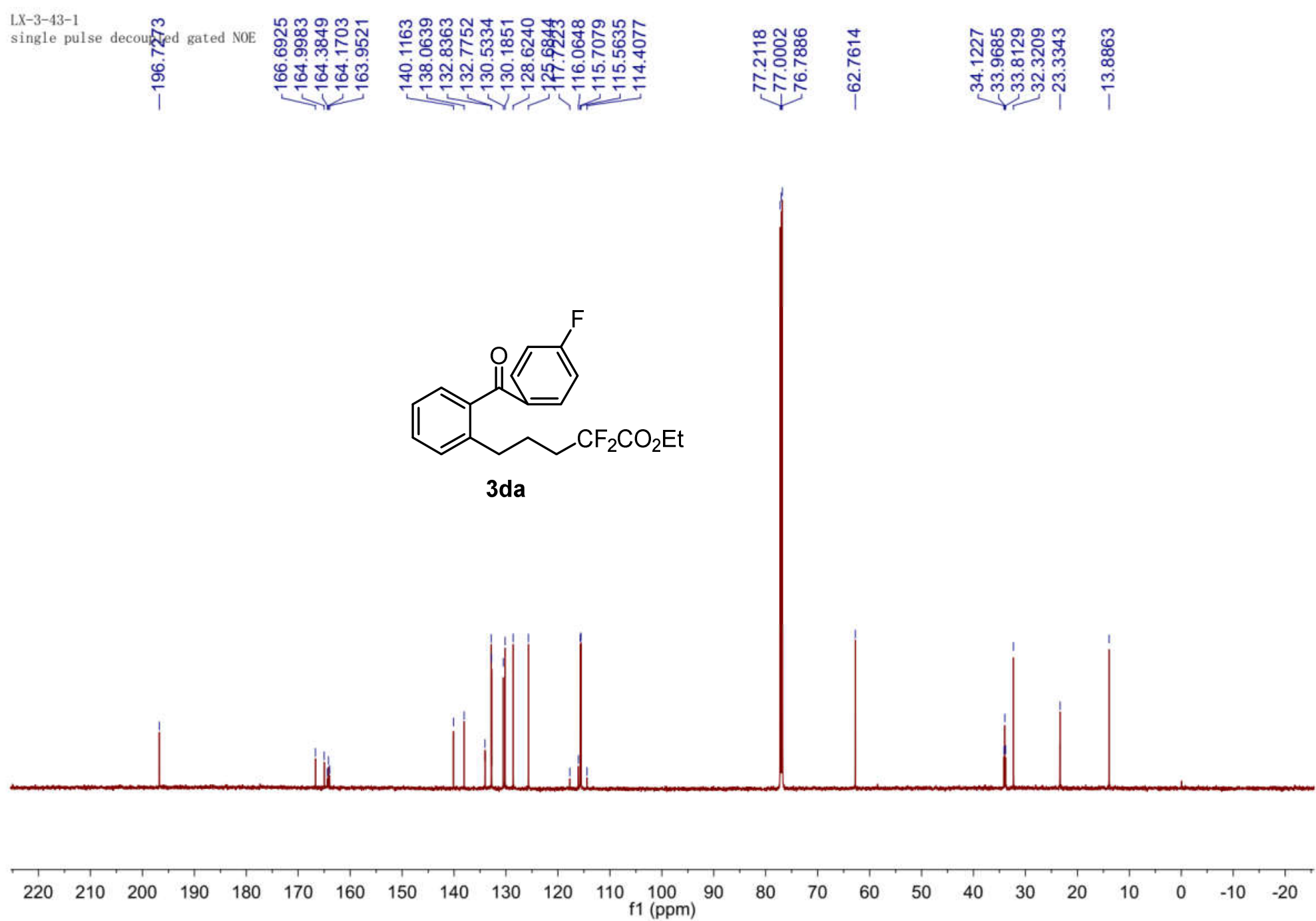



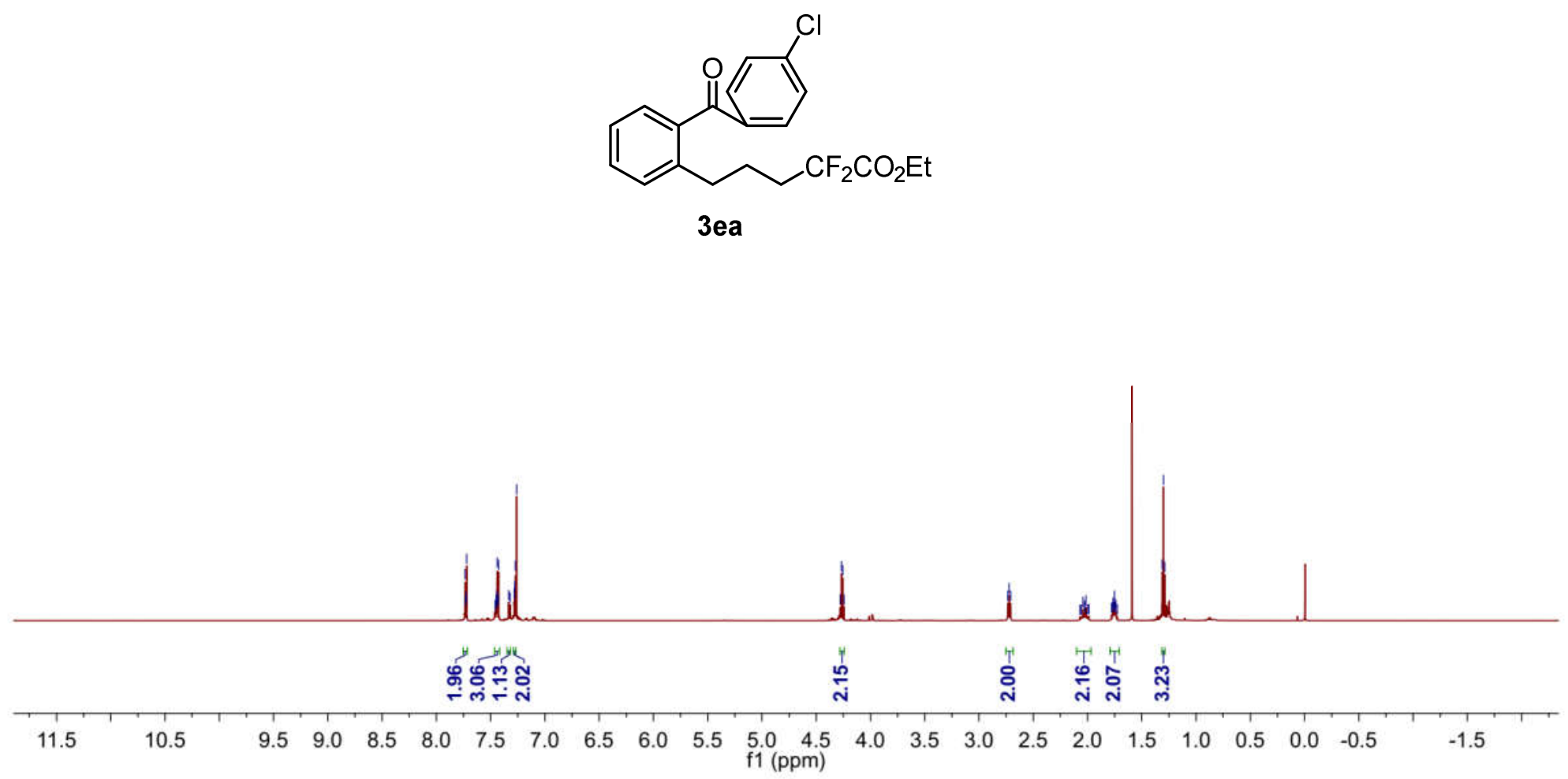


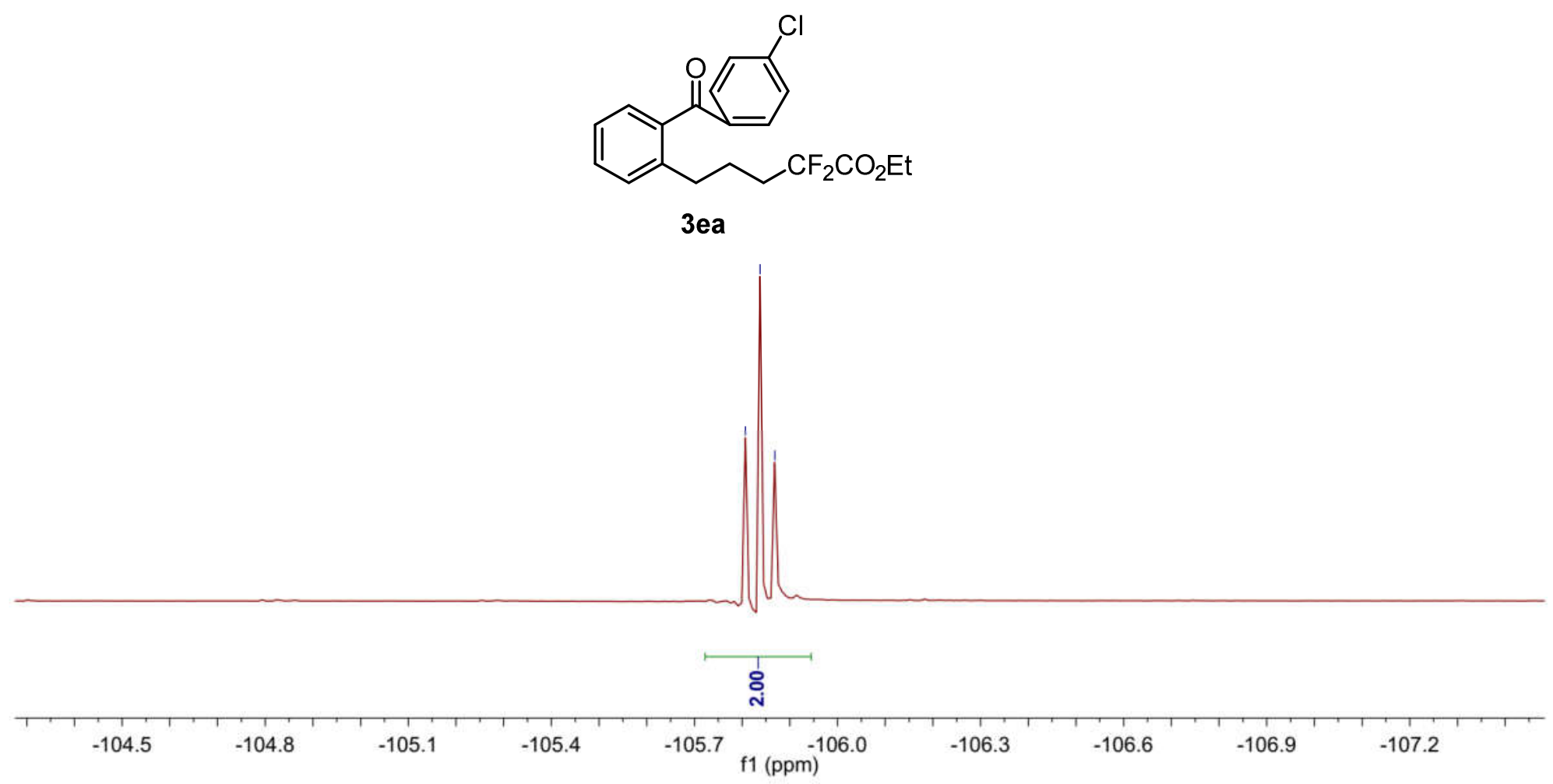




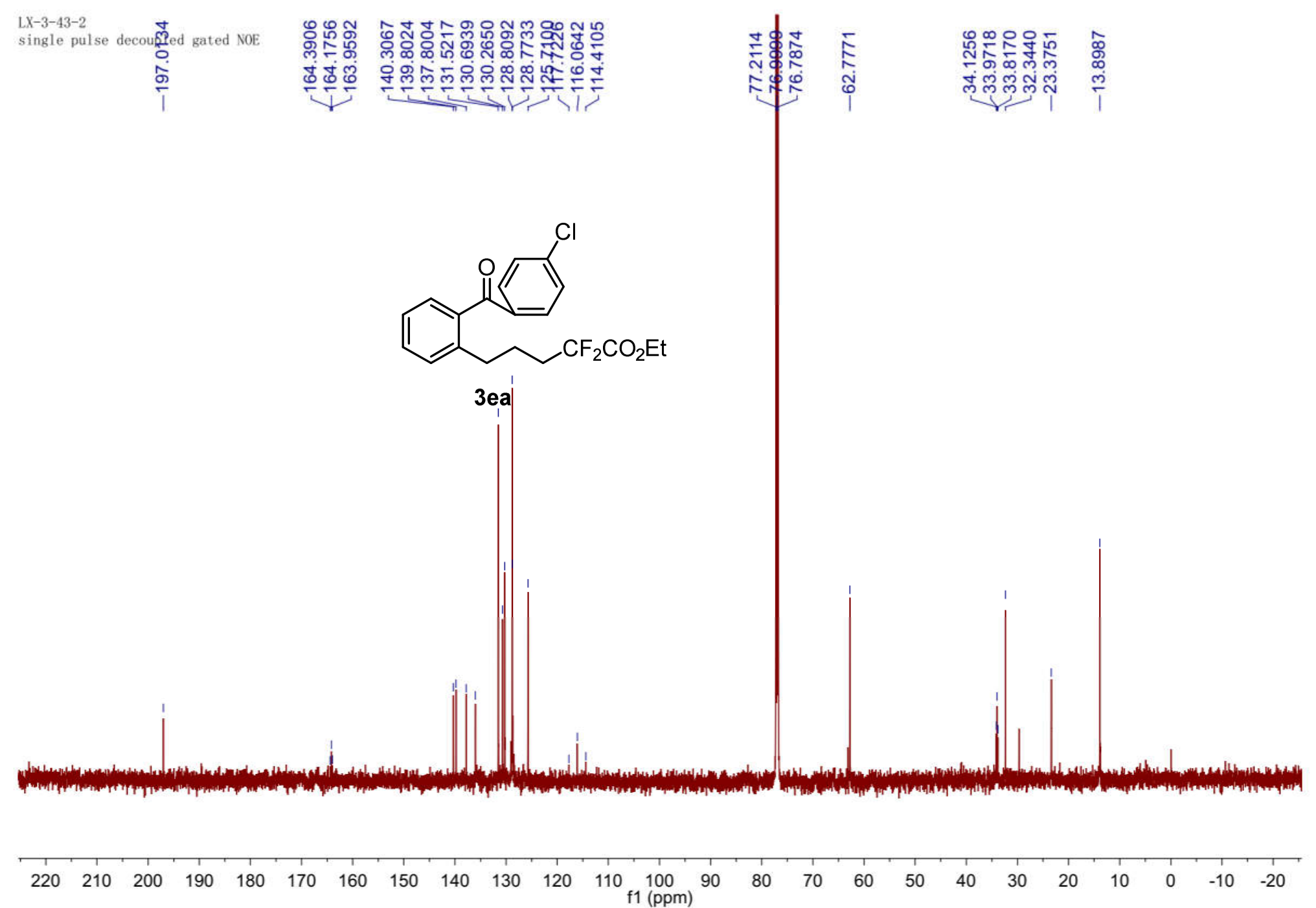




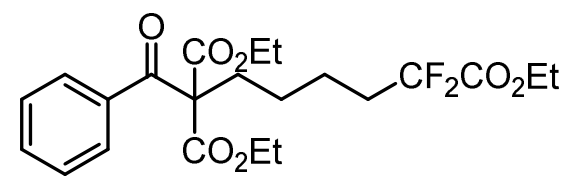

$3 \mathrm{fa}$

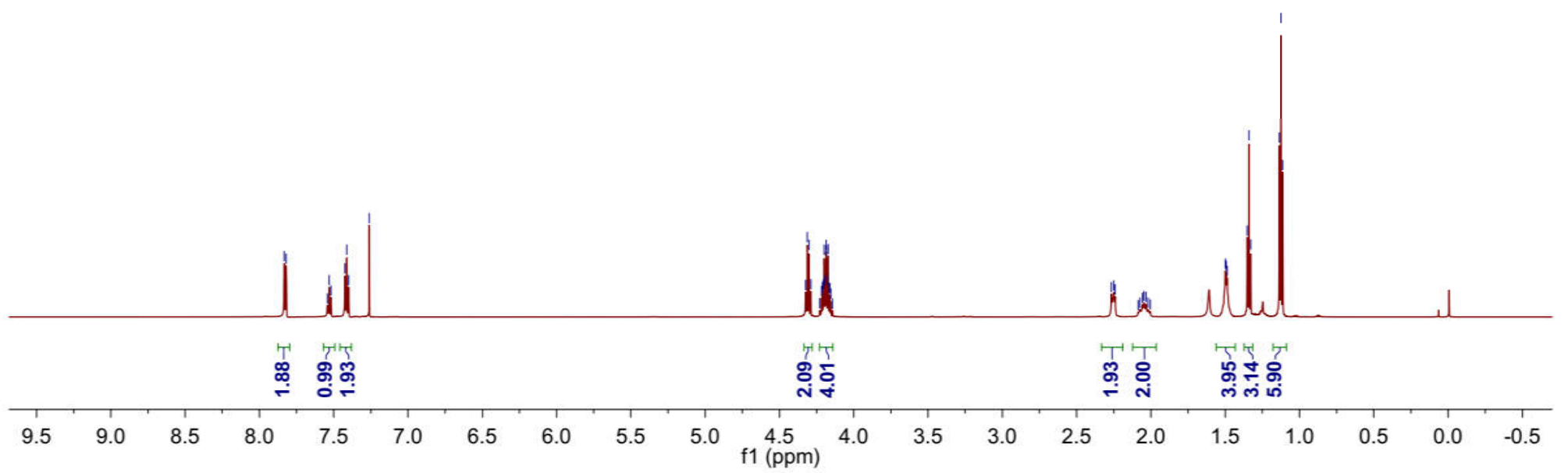


(1)

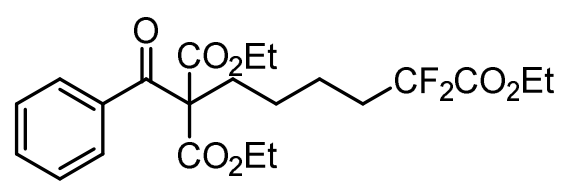

$3 \mathrm{fa}$

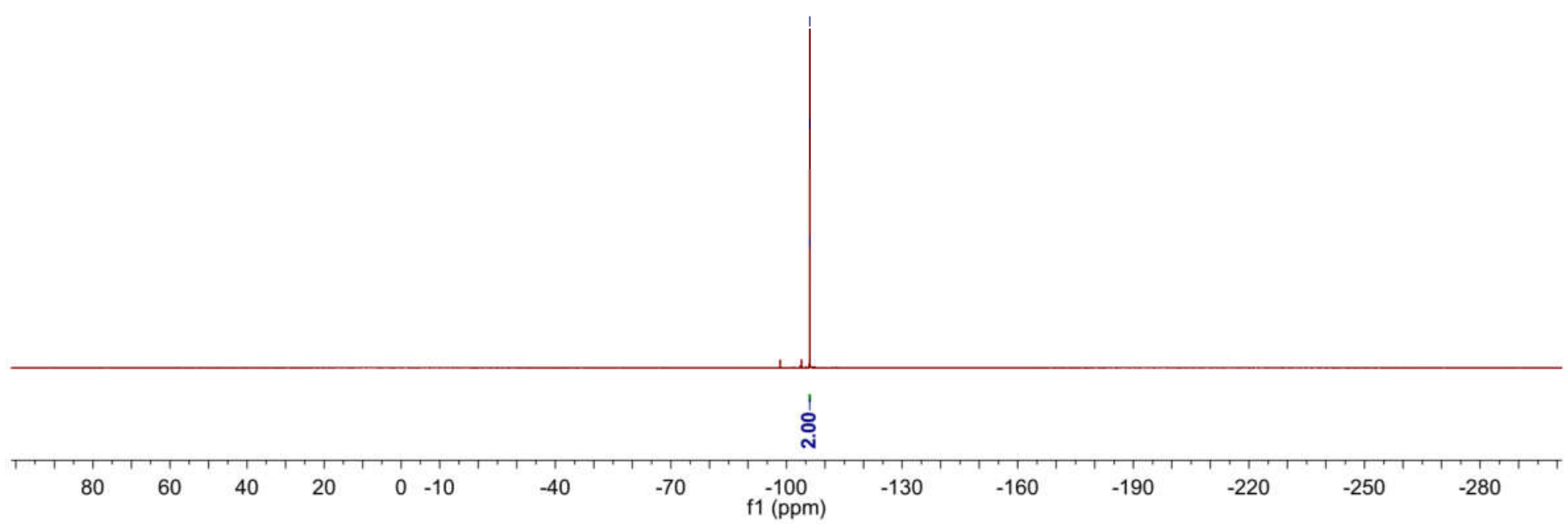




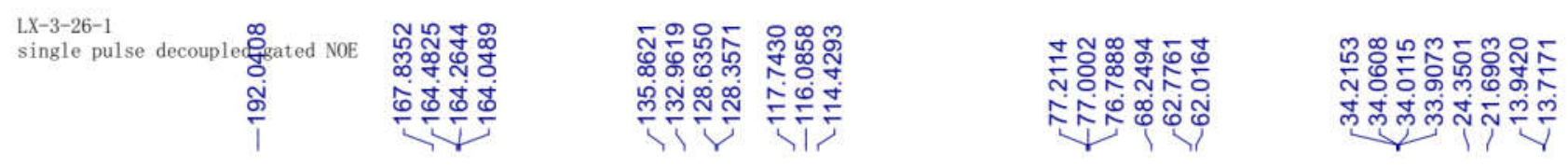

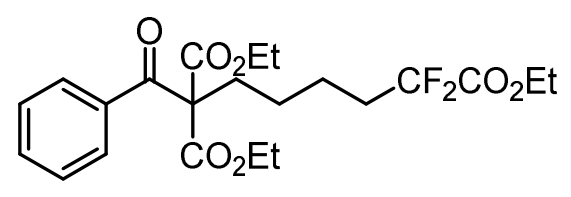

$3 f a$

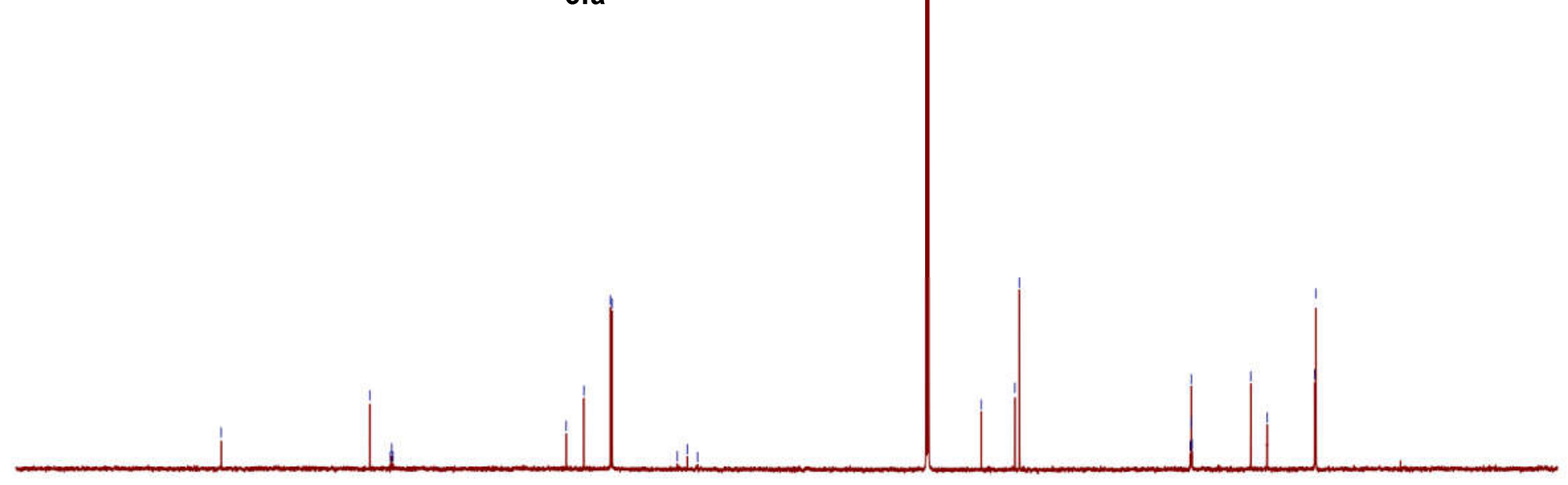

$\begin{array}{lllllllllllllllllllllllllllllllllll}220 & 210 & 200 & 190 & 180 & 170 & 160 & 150 & 140 & 130 & 120 & 110 & 100 & 90 & 80 & 70 & 60 & 50 & 40 & 30 & 20 & 10 & 0 & -10 & -20\end{array}$ 


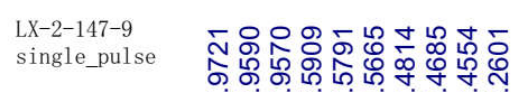

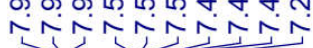

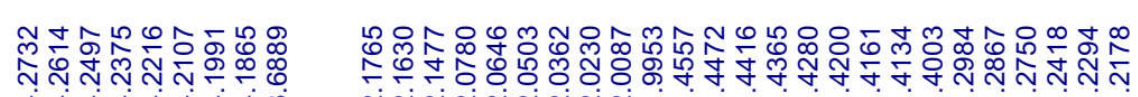

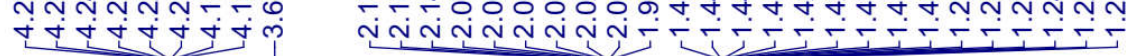

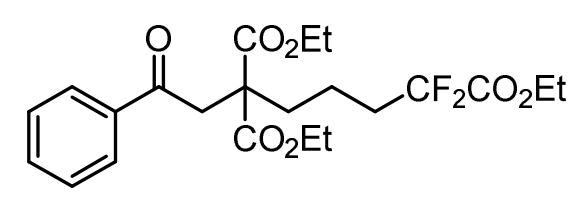

3 ga

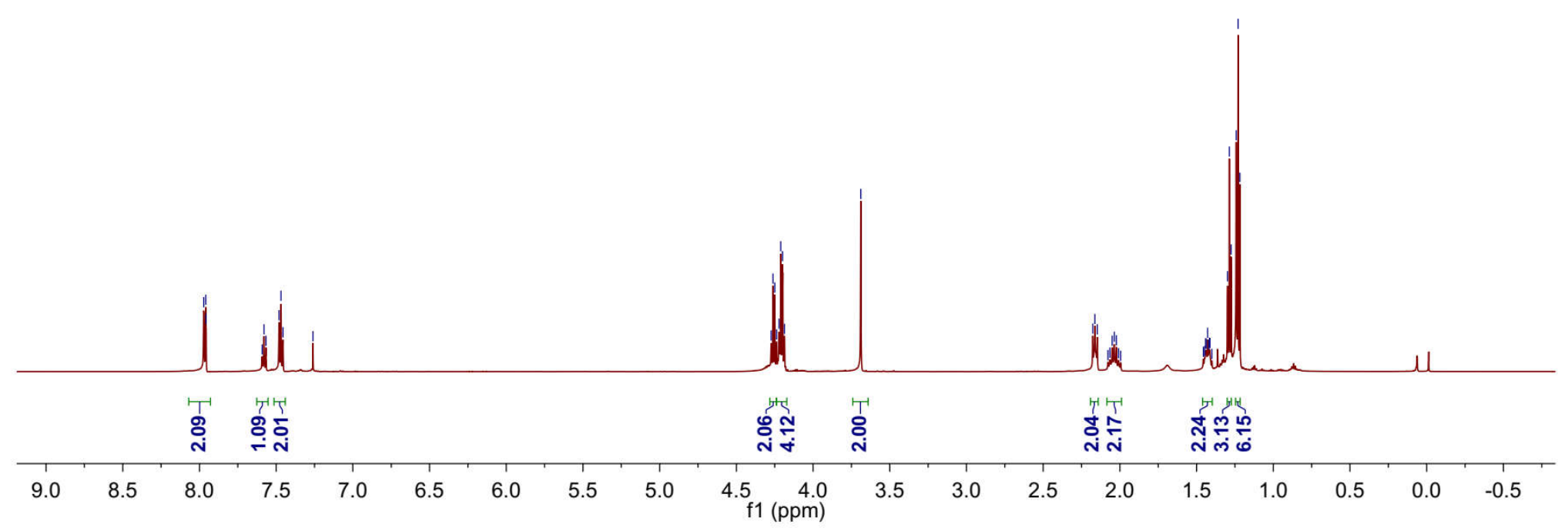


LY-2-1-1

single_puls

둥응

운우

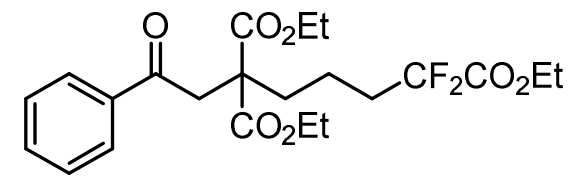

3ga

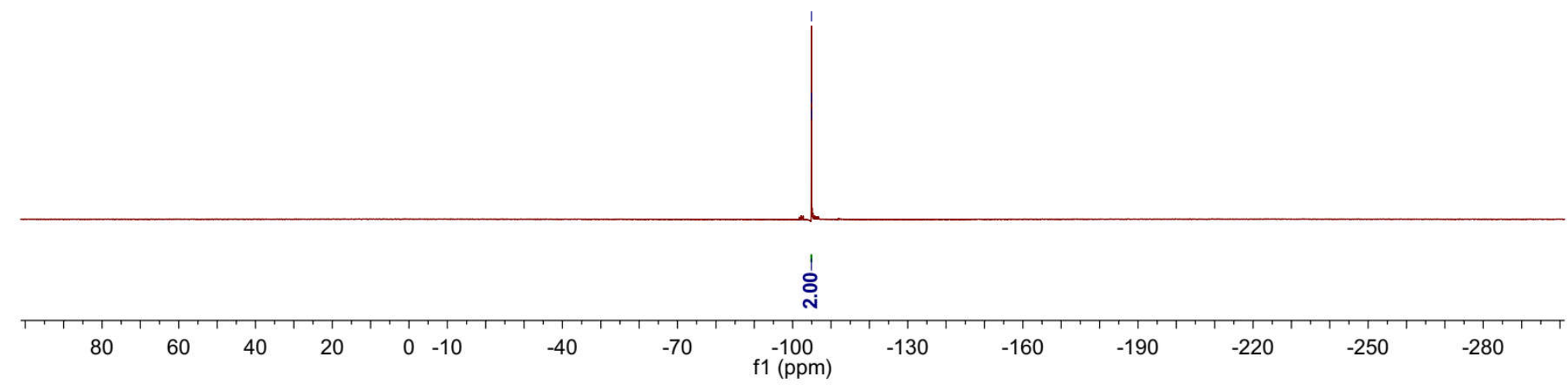



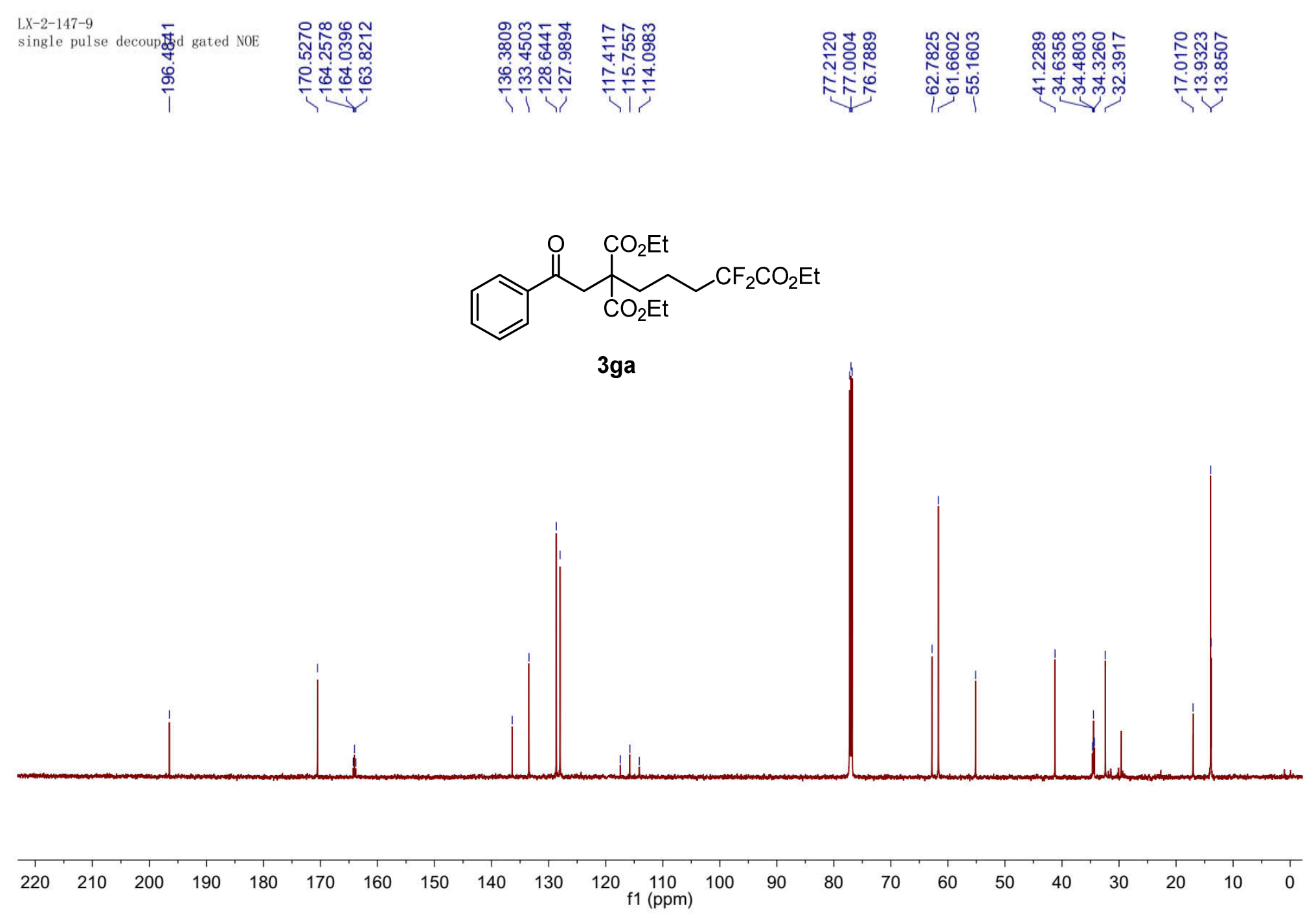


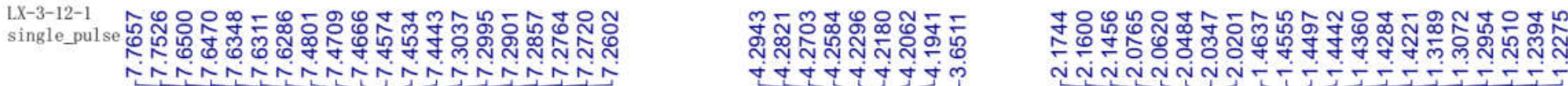

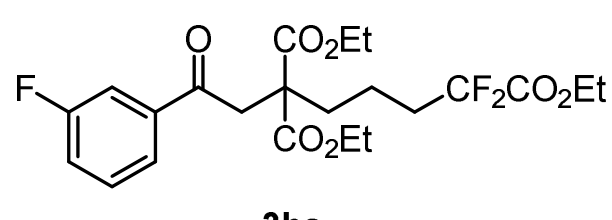

3ha

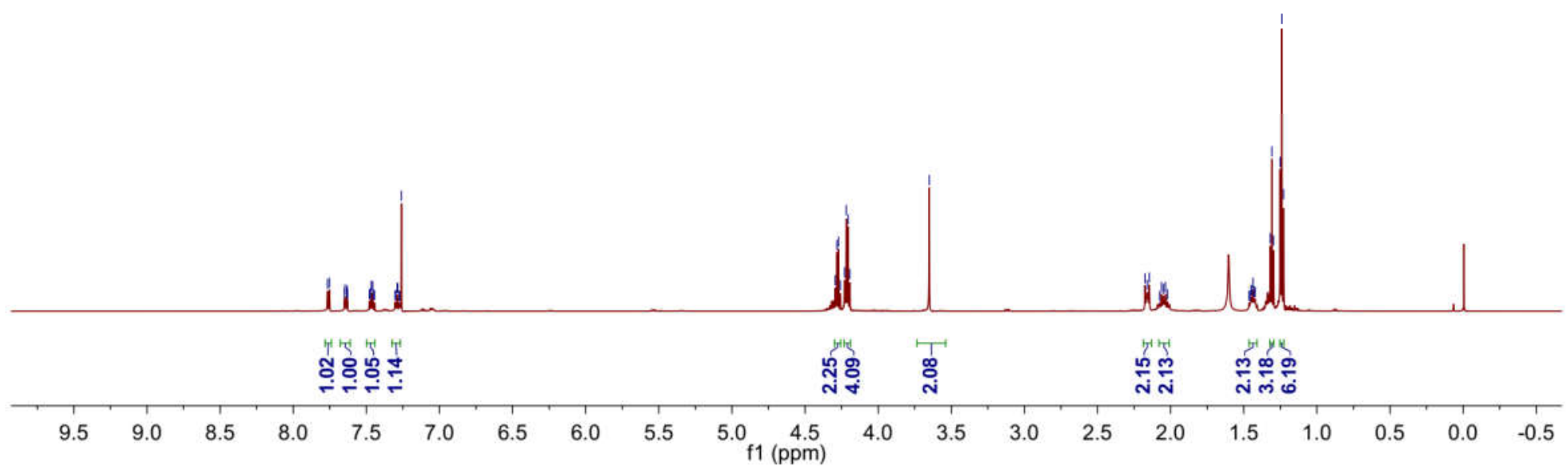




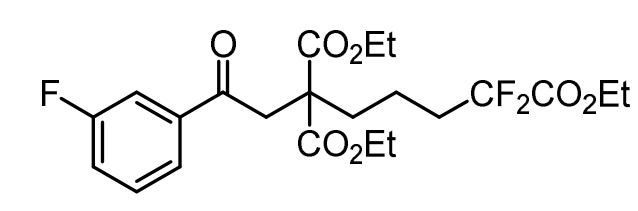

3ha

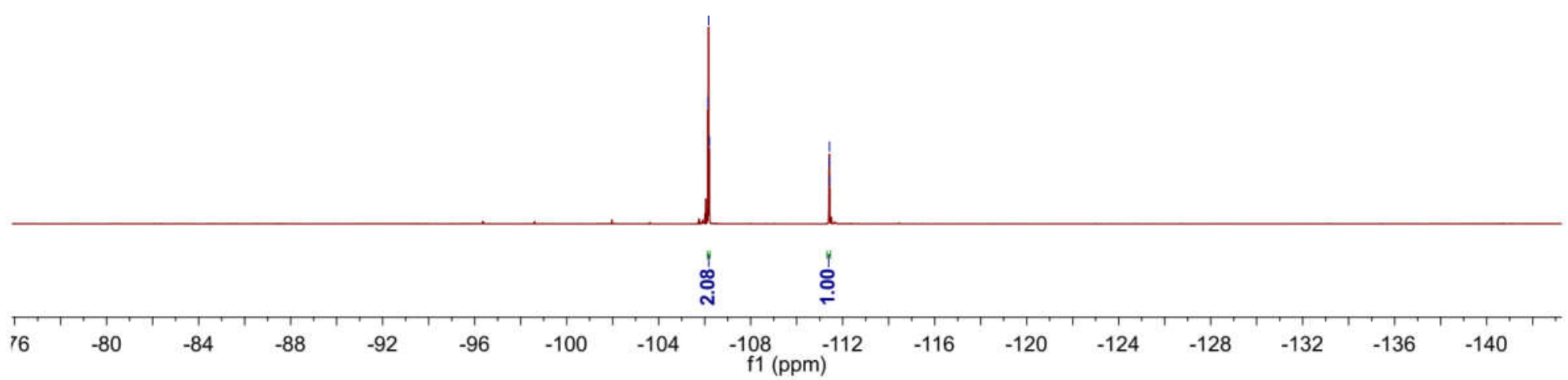




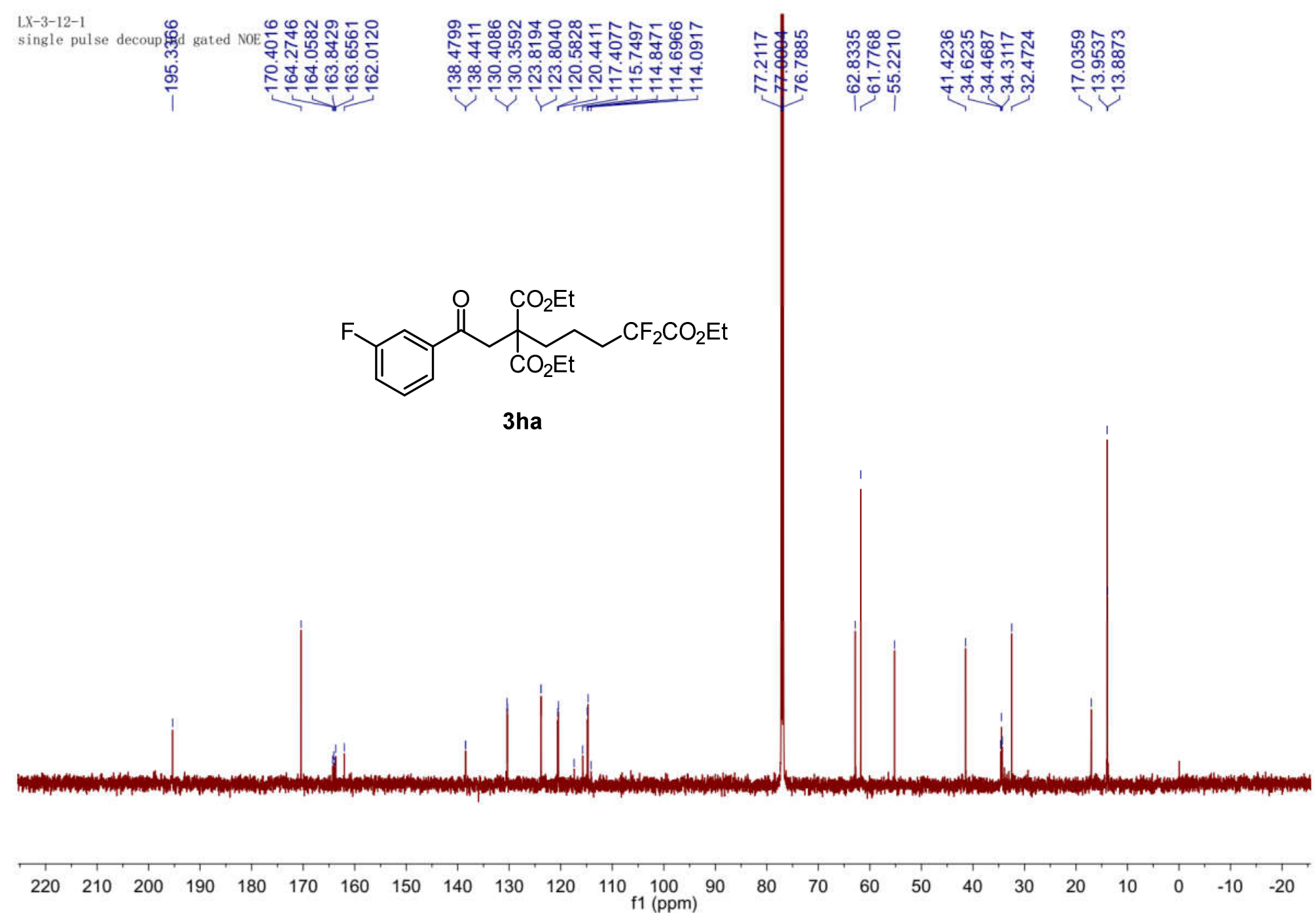




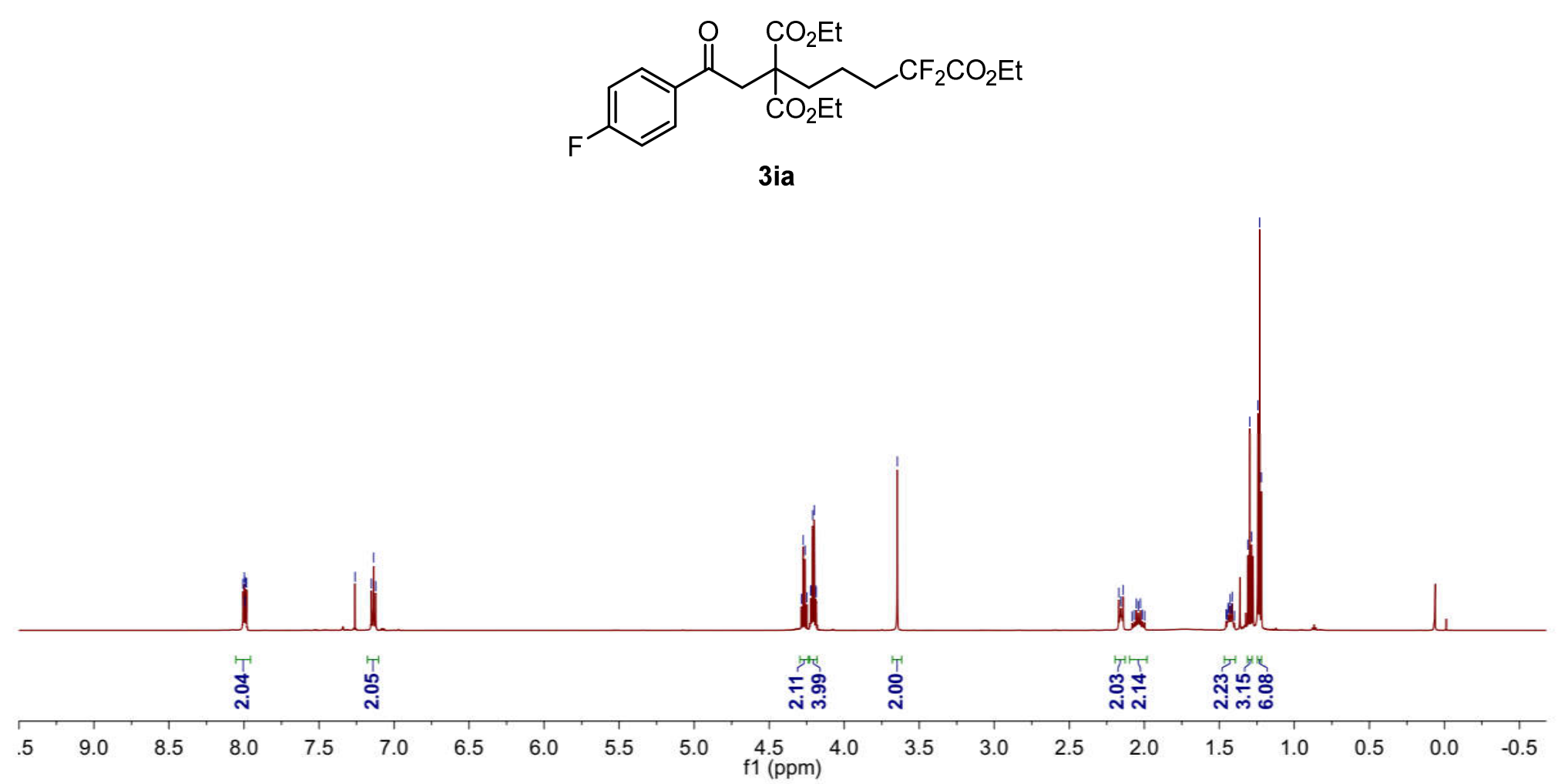




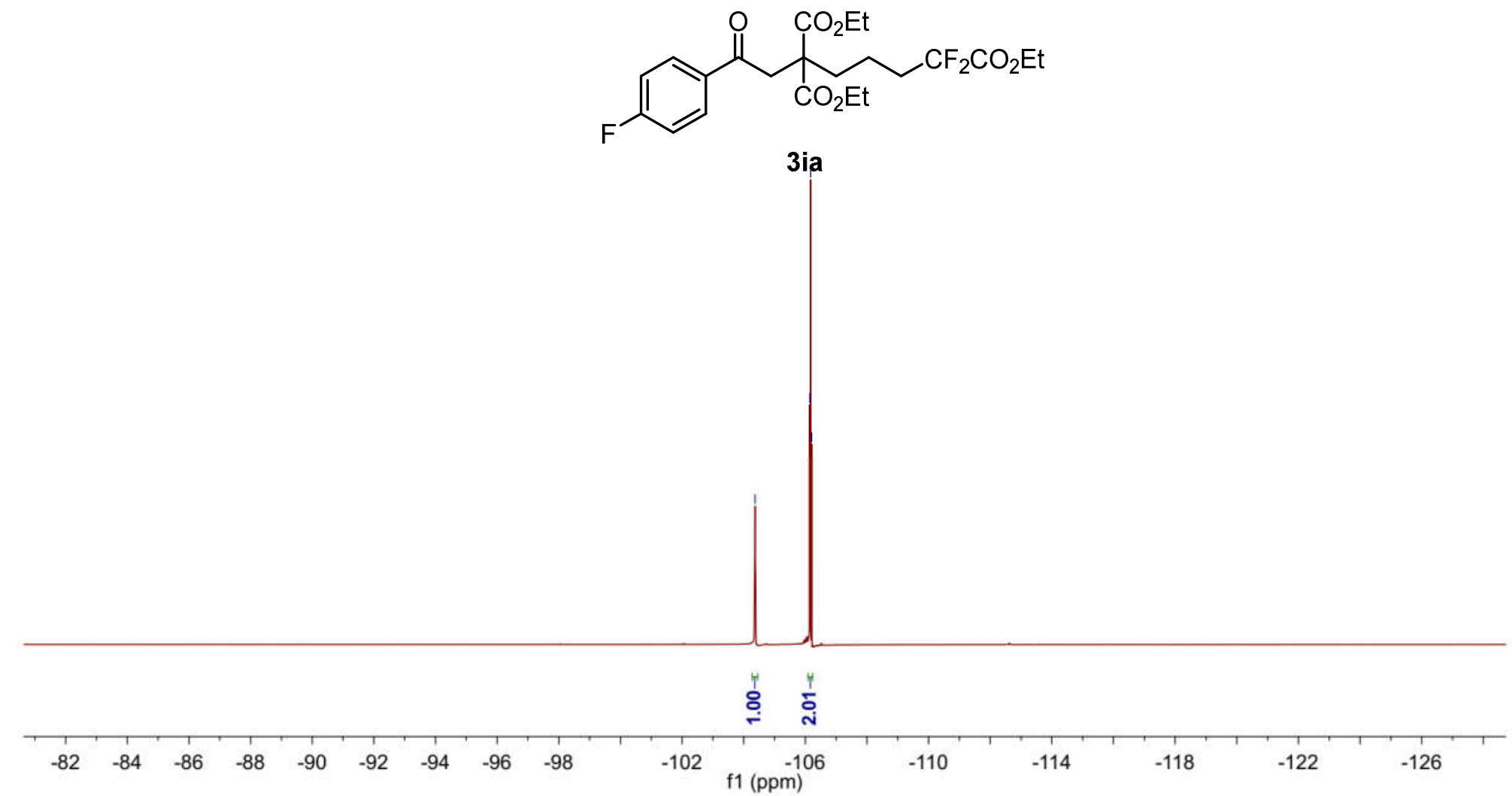



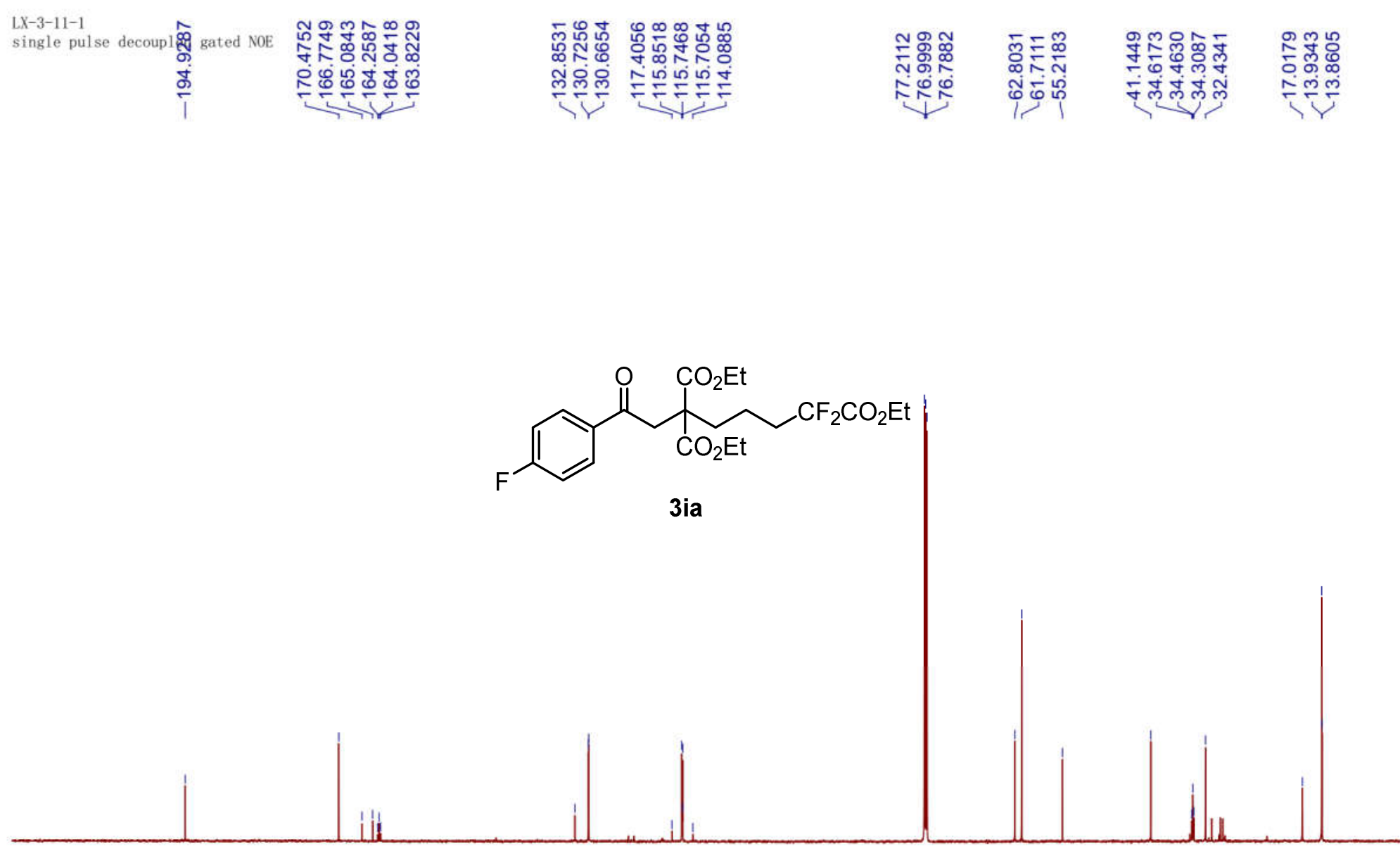

$\begin{array}{llllllllllllllllllllllllll}220 & 210 & 200 & 190 & 180 & 170 & 160 & 150 & 140 & 130 & 120 & 110 & 100 & 90 & 80 & 70 & 60 & 50 & 40 & 30 & 20 & 10\end{array}$ 


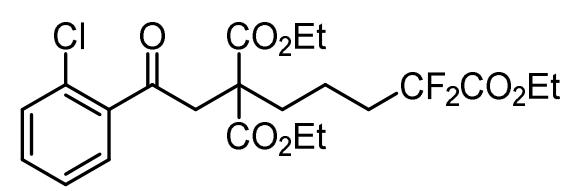

$3 j a$

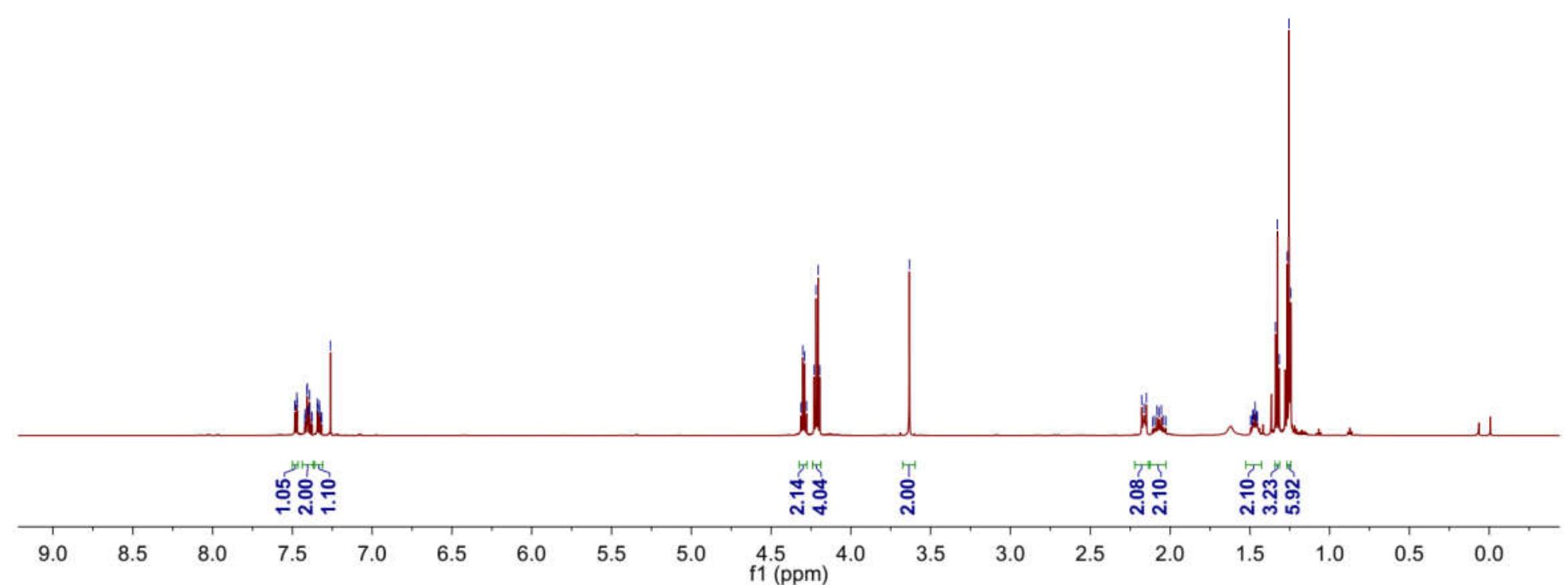


LX-3-8-1

single_puls

钽志总

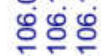

운

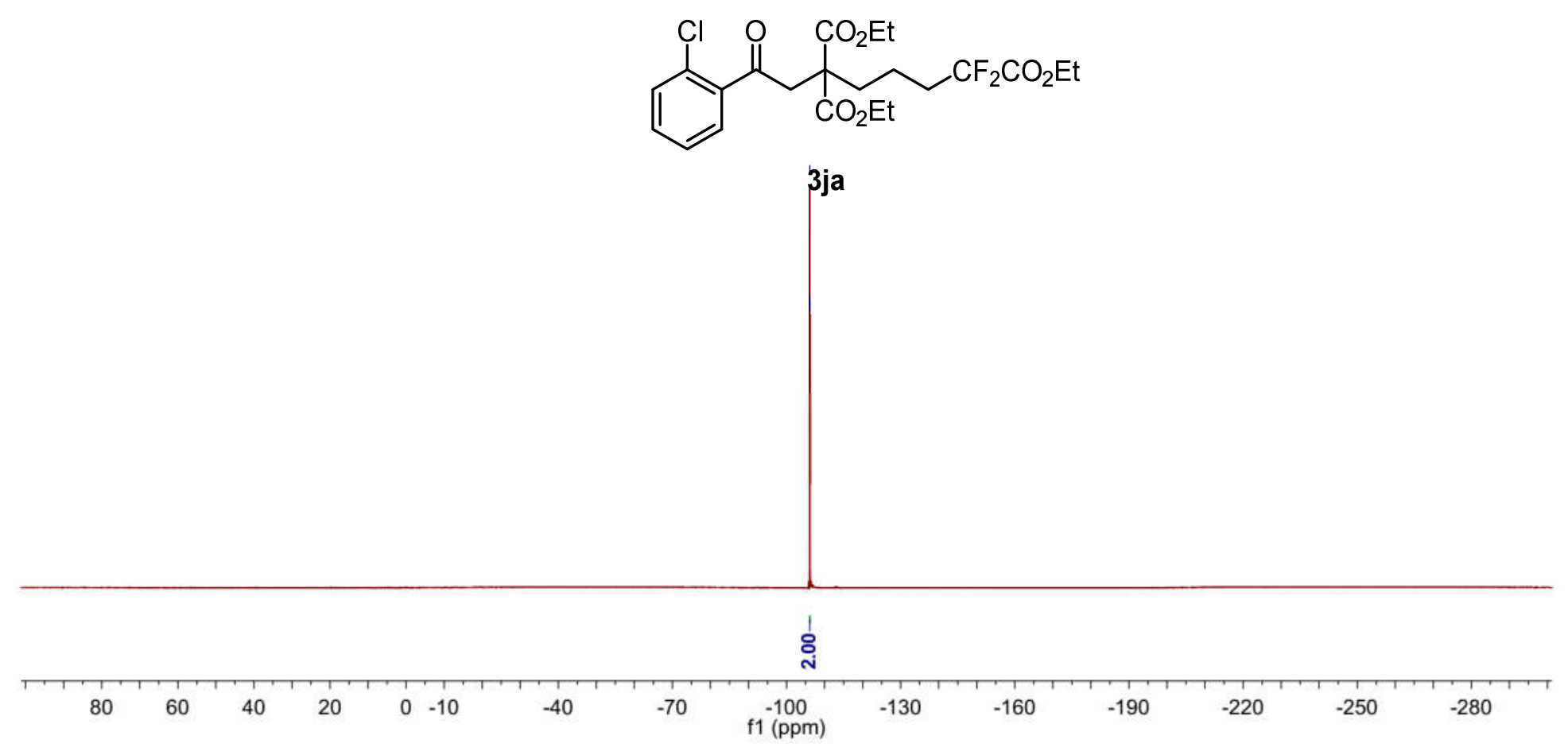




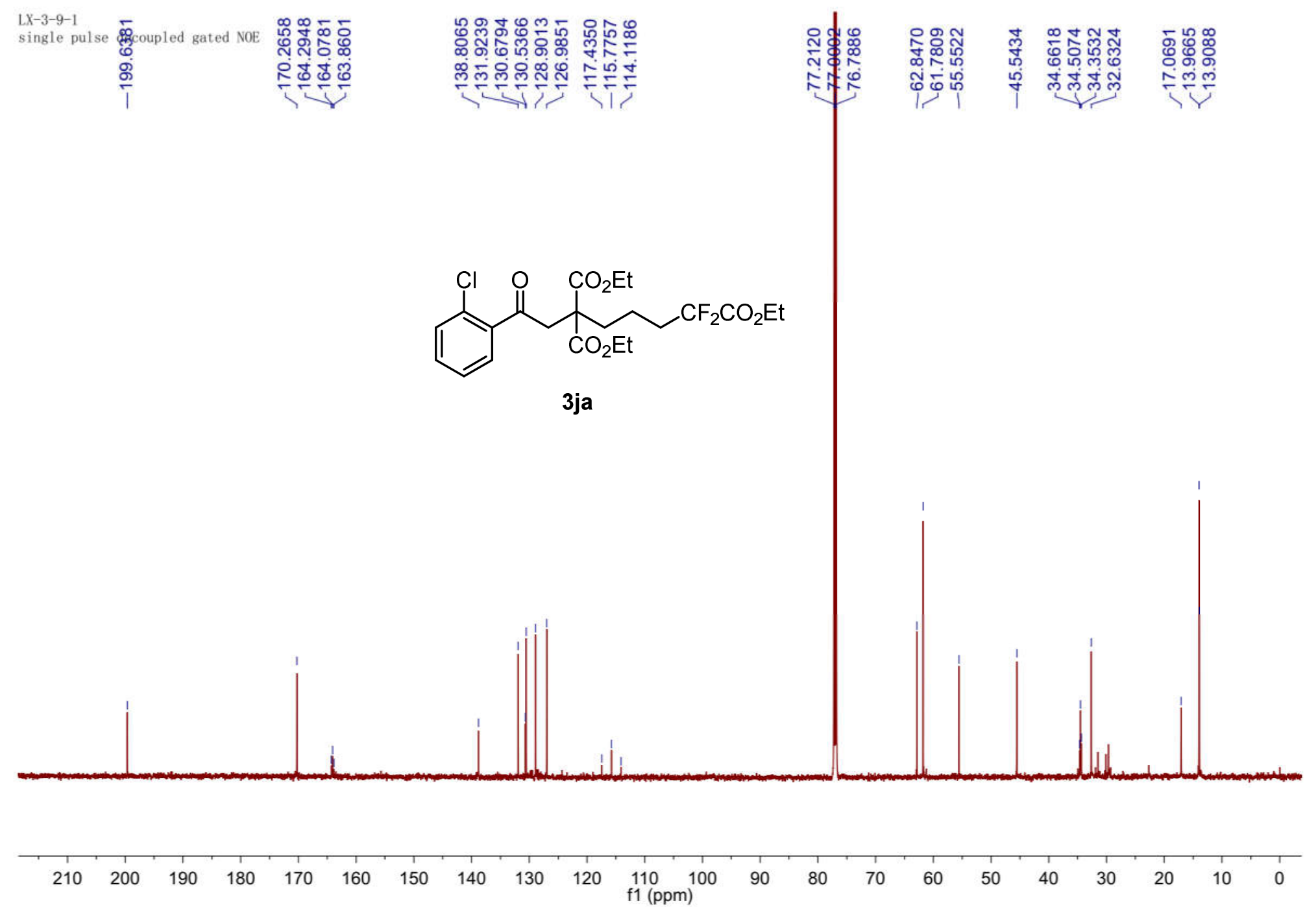



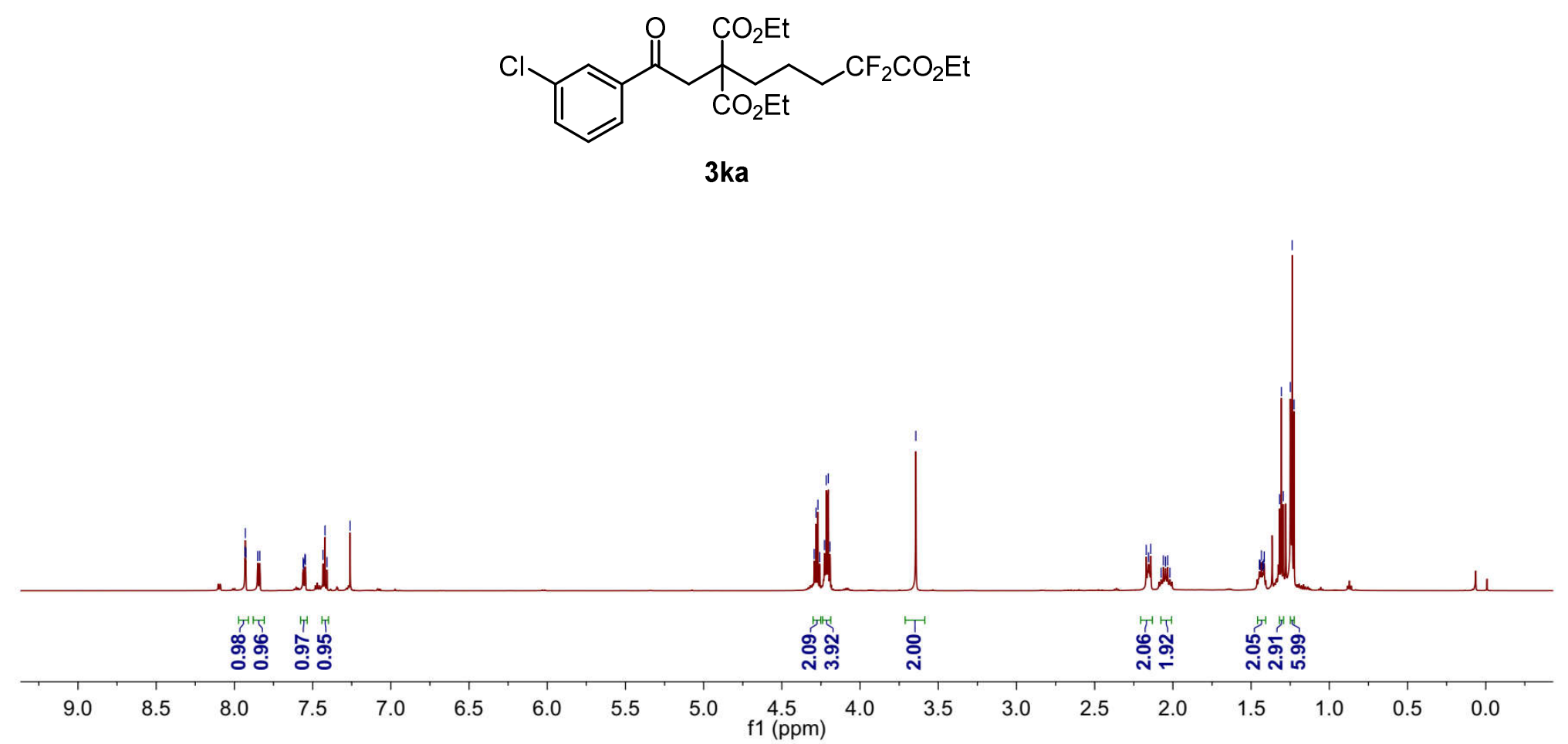
LX-3-8-2

single_pulse

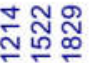

$8 \%$

i

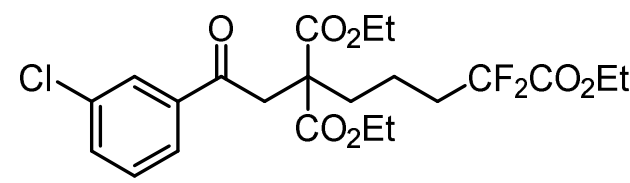

3ka

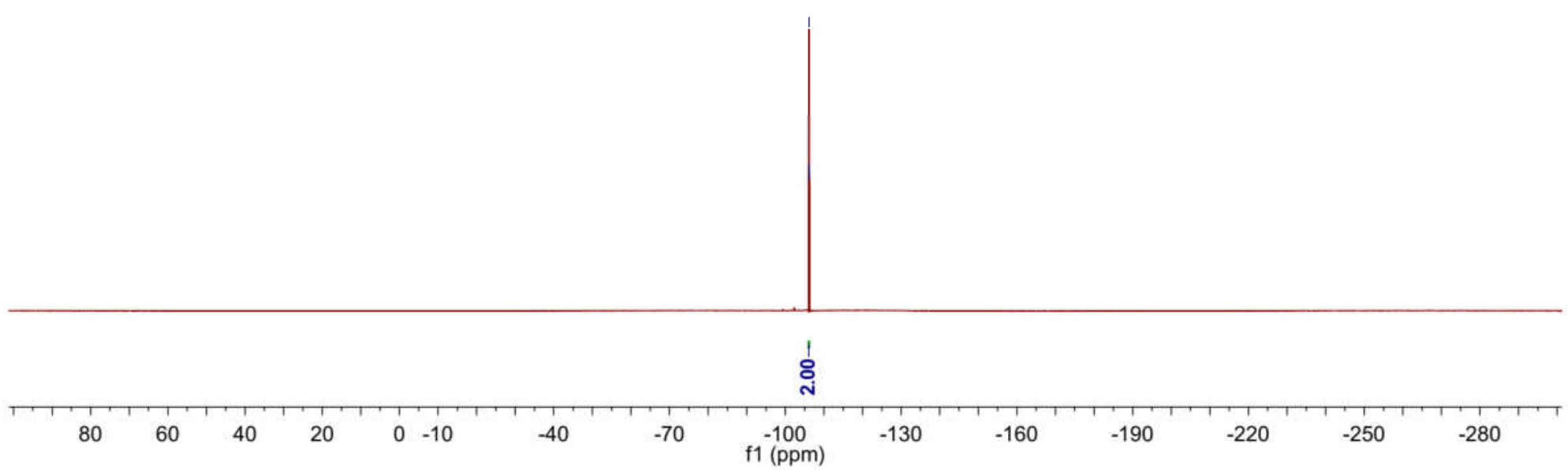




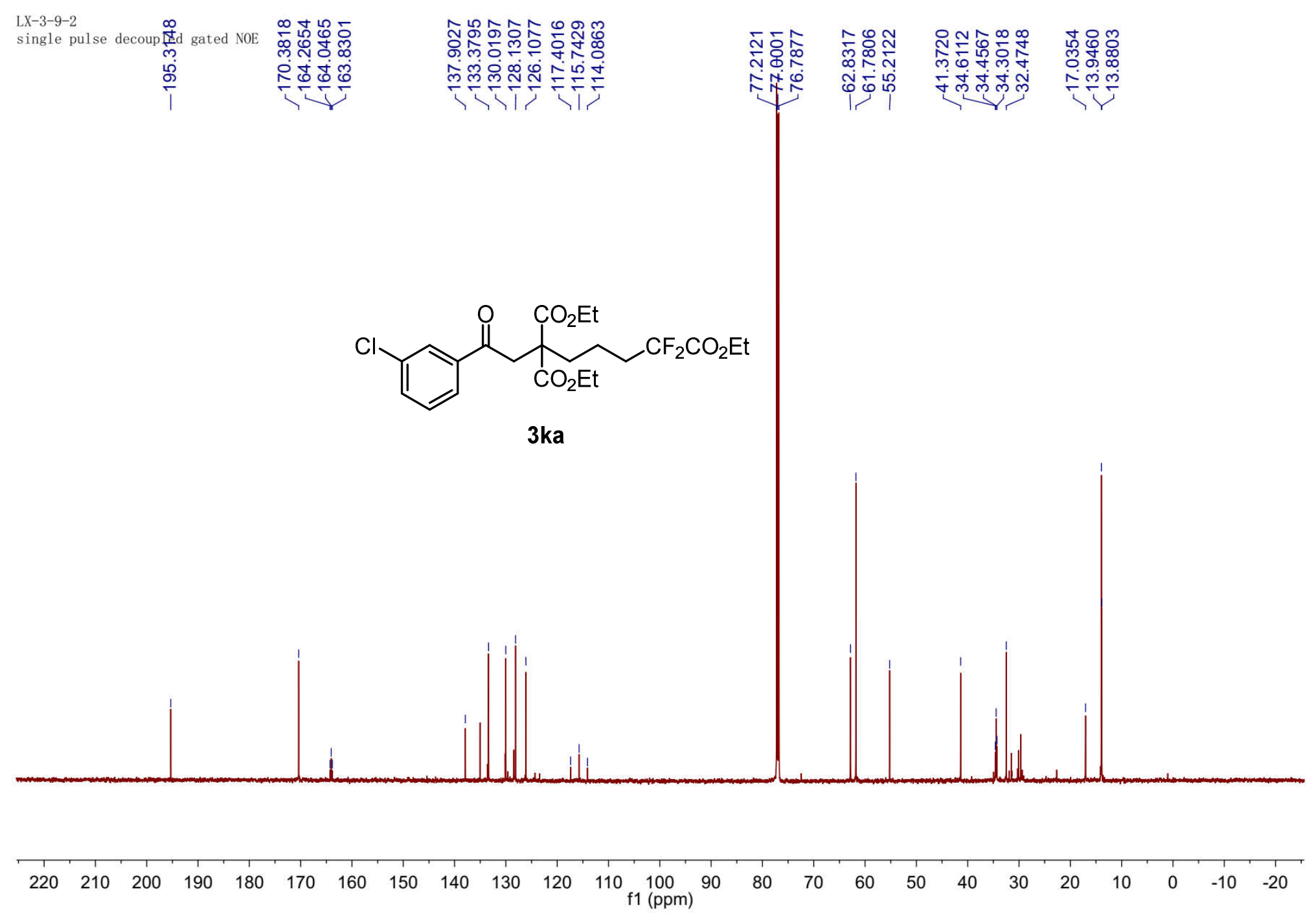




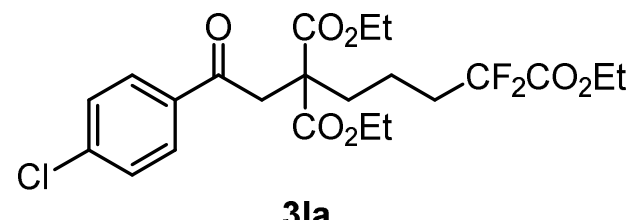

$3 l a$

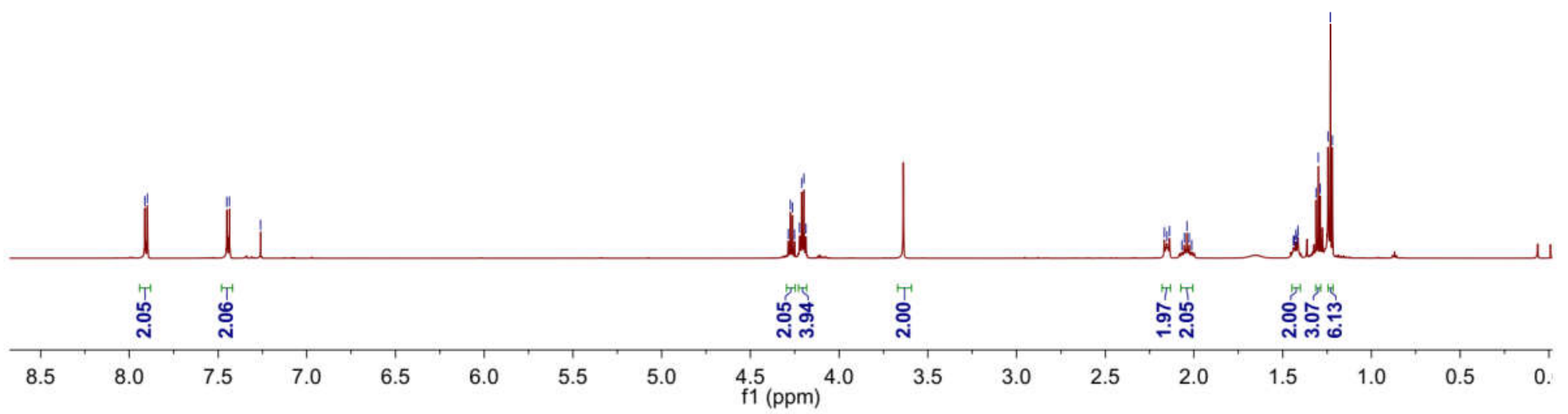




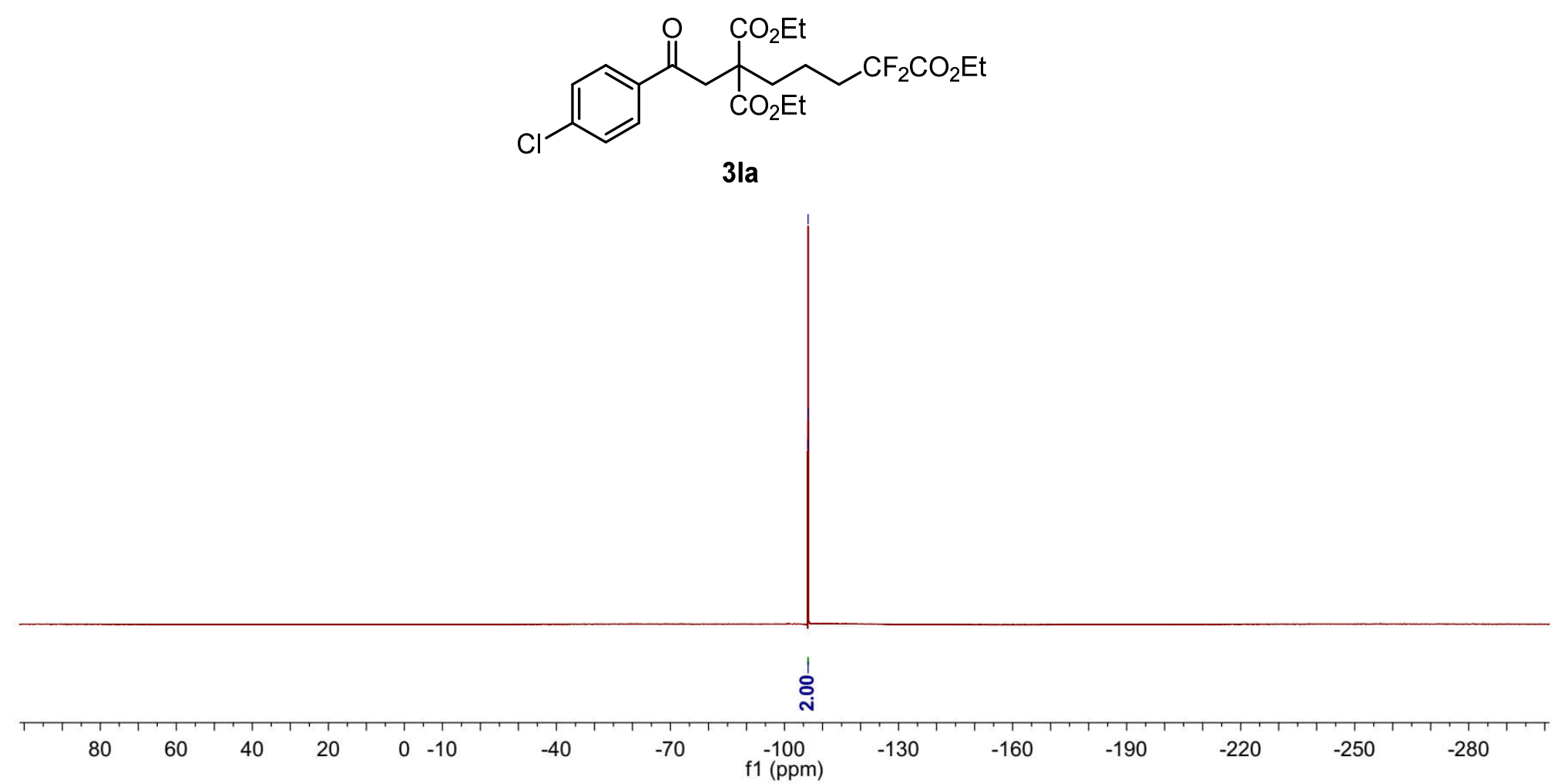




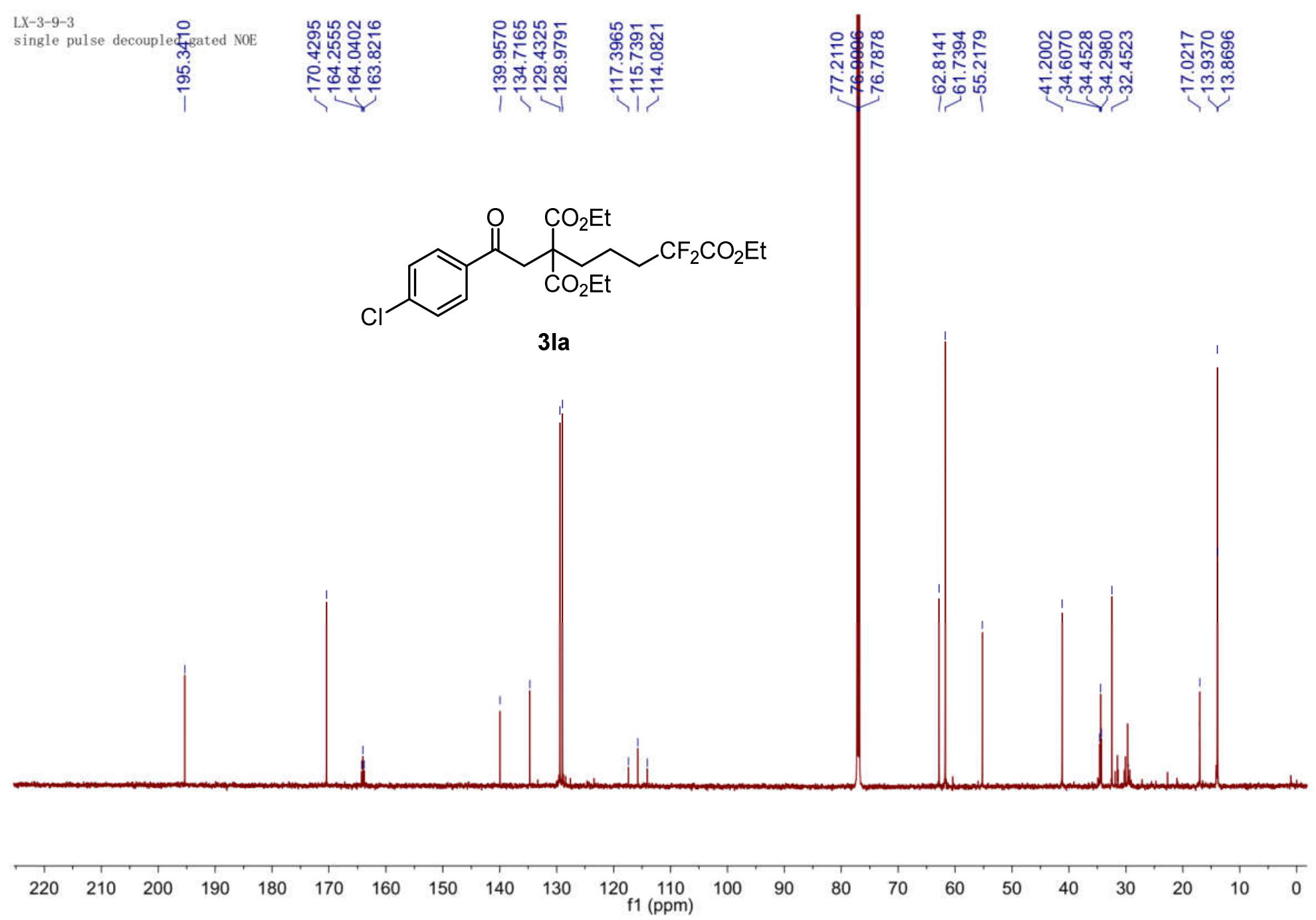



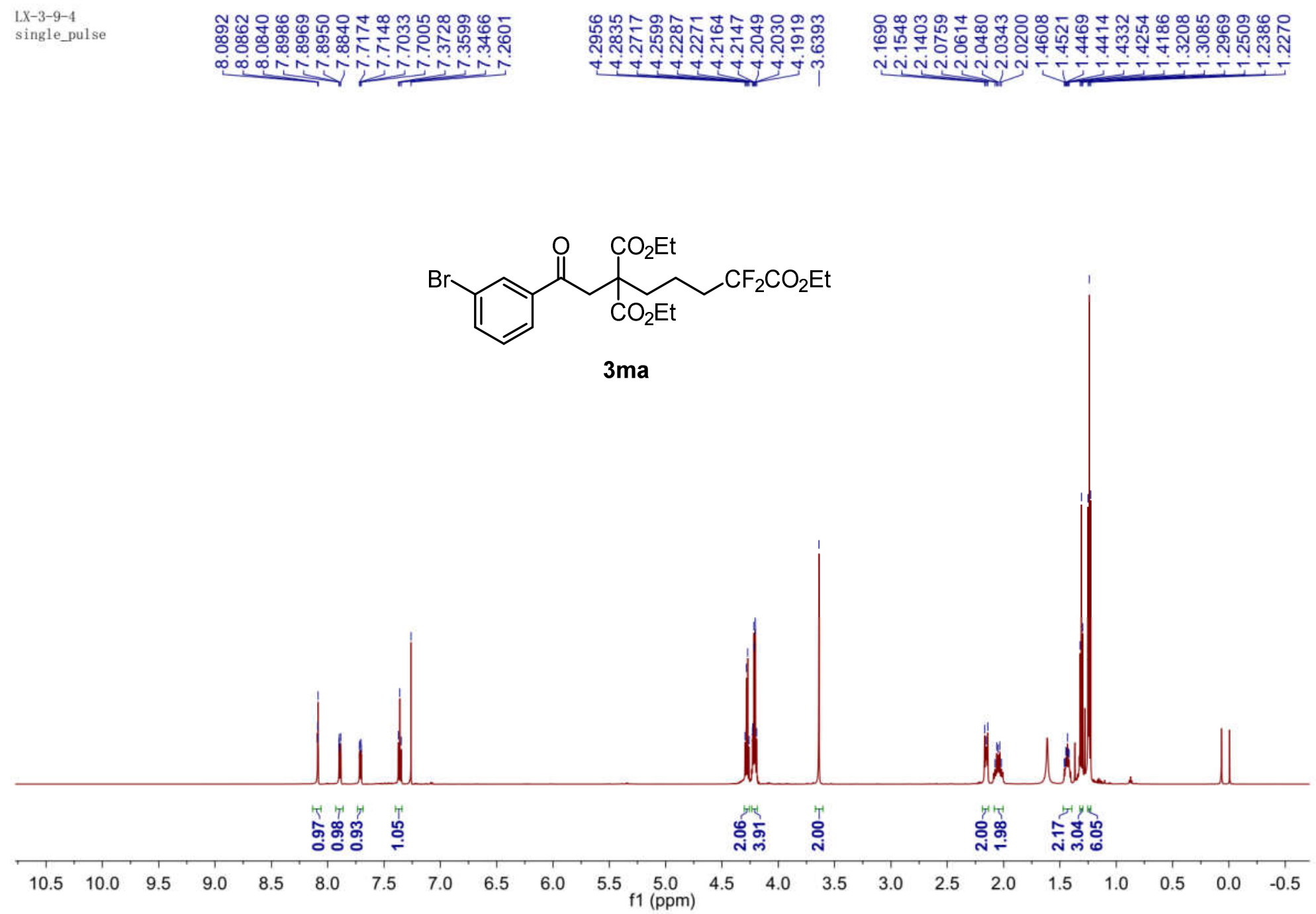


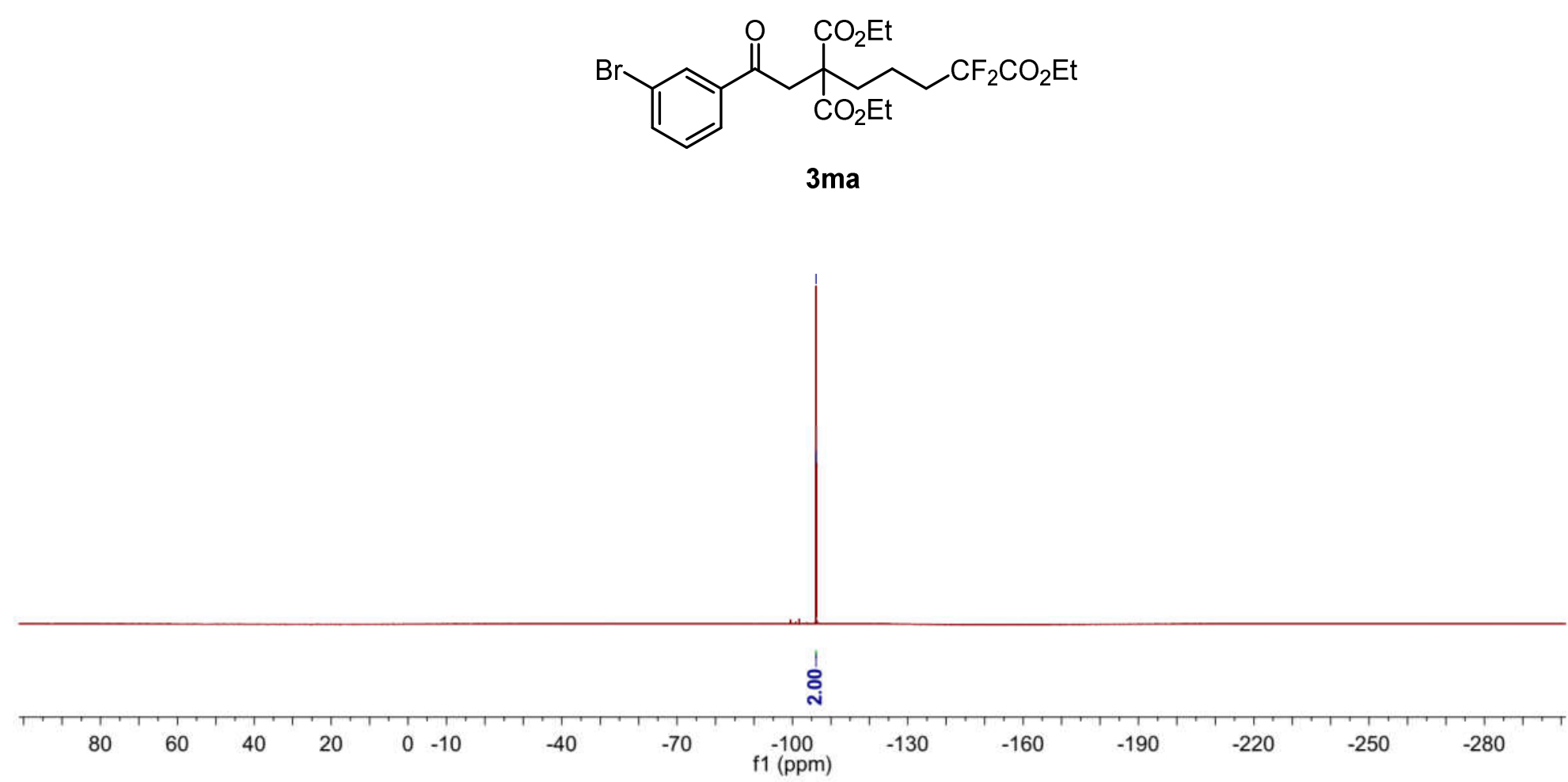




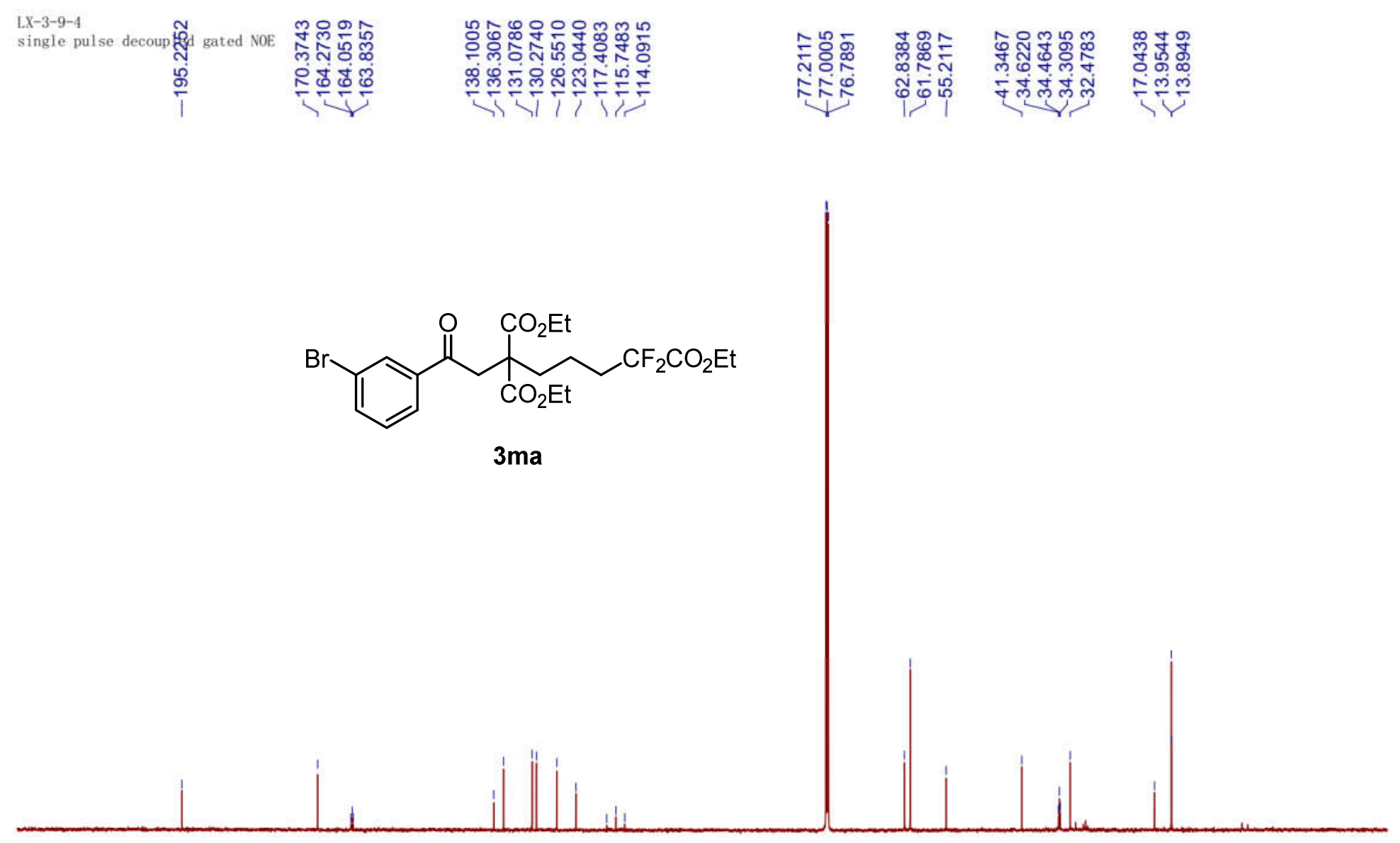

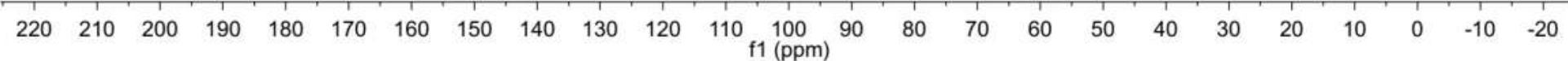



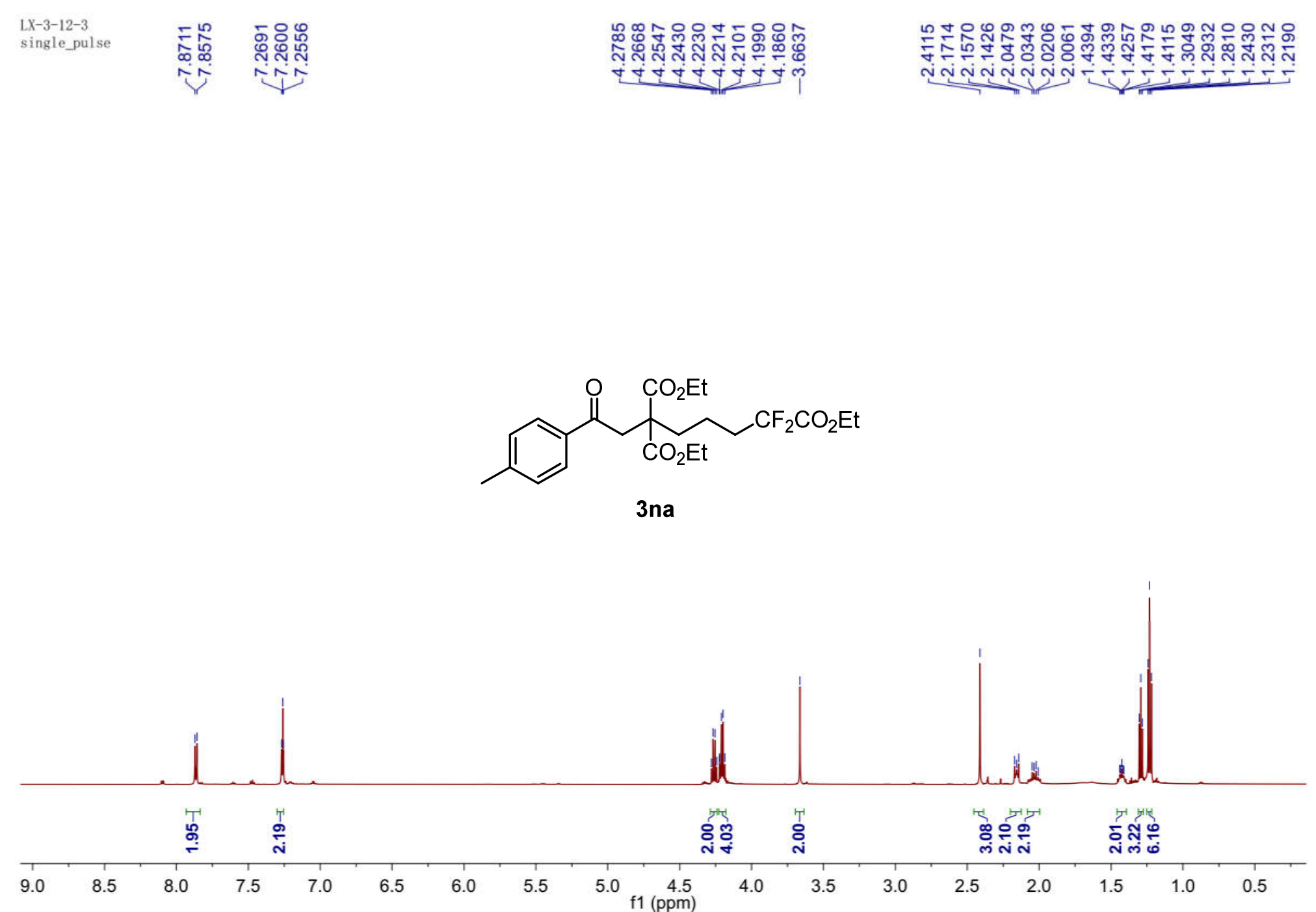
LX-3-12-3

single_puls

홍으요

$\Leftrightarrow 8$

운

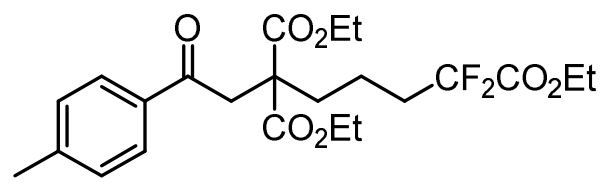

3na

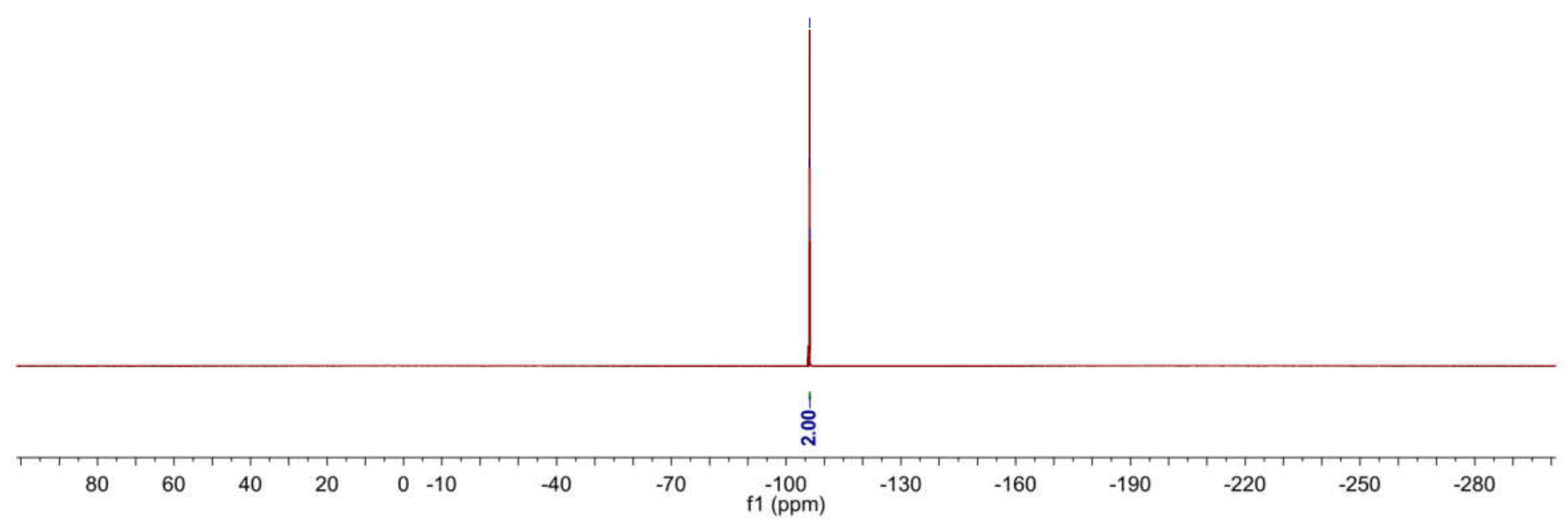




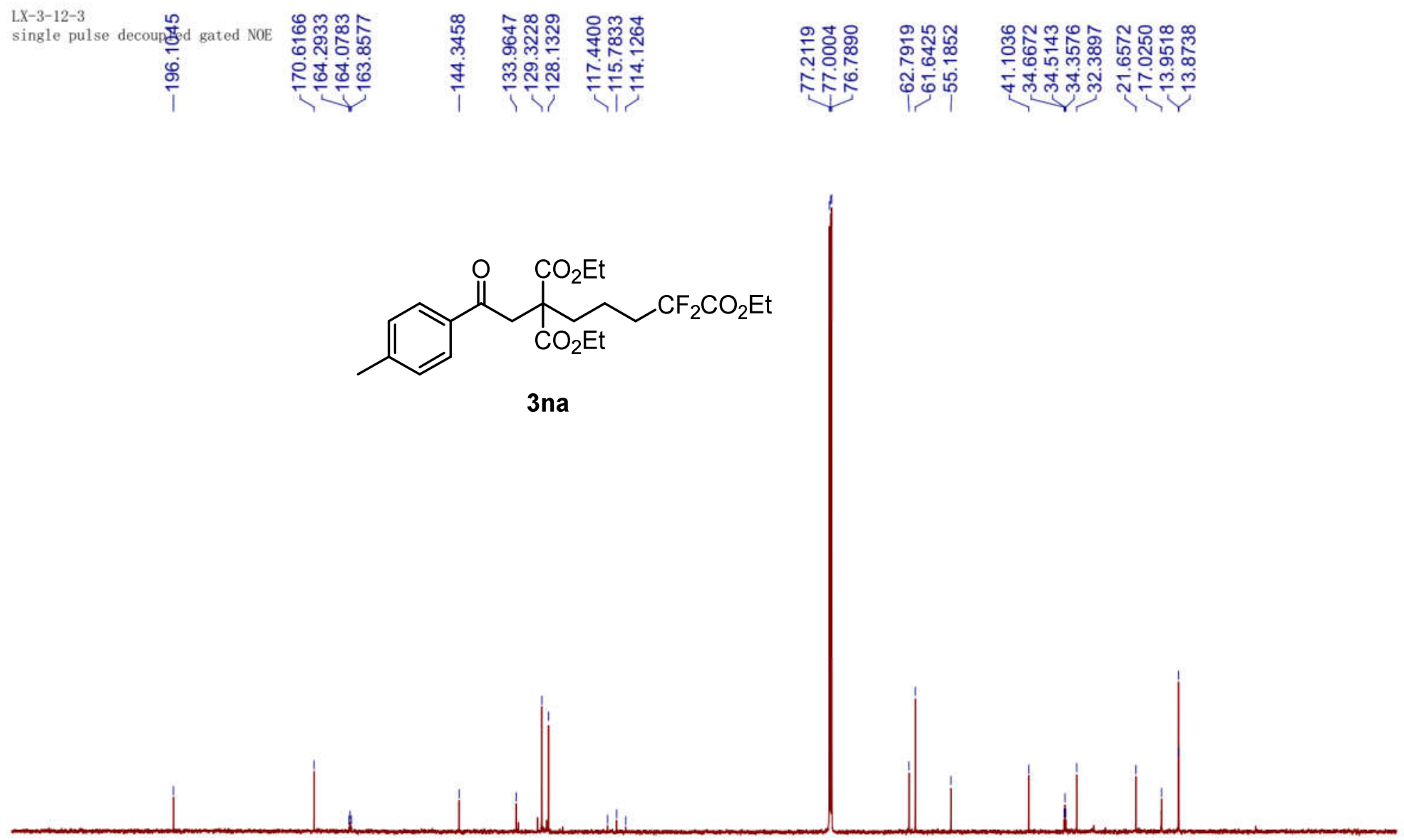

$\begin{array}{llllllllllllllllllllllllllllll}220 & 210 & 200 & 190 & 180 & 170 & 160 & 150 & 140 & 130 & 120 & 110 & \begin{array}{c}100 \\ \mathrm{f} 1(\mathrm{ppm})\end{array} & 90 & 80 & 70 & 60 & 50 & 40 & 30 & 20 & 10 & 0 & -10 & -20\end{array}$ 

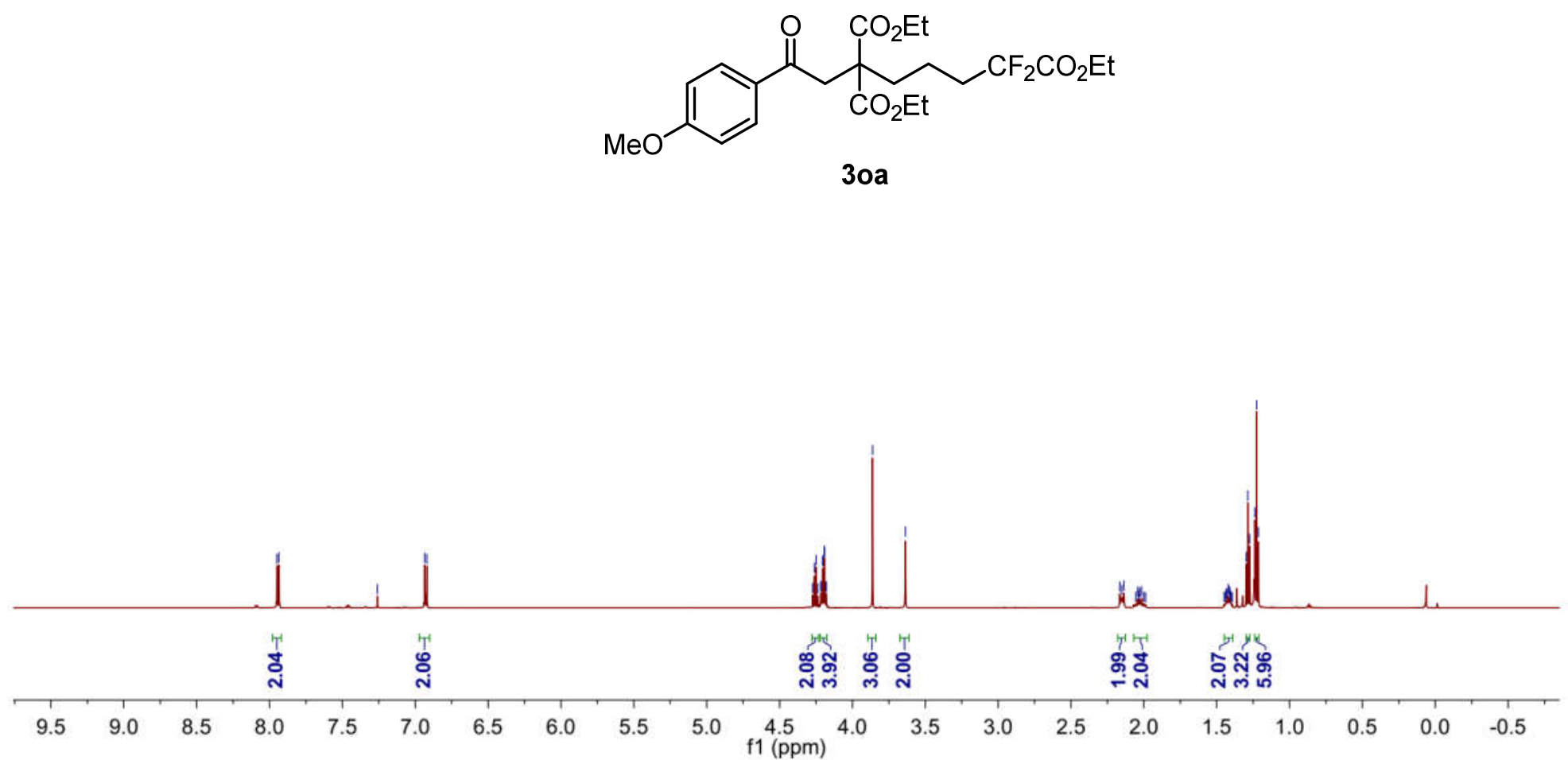
웅

广

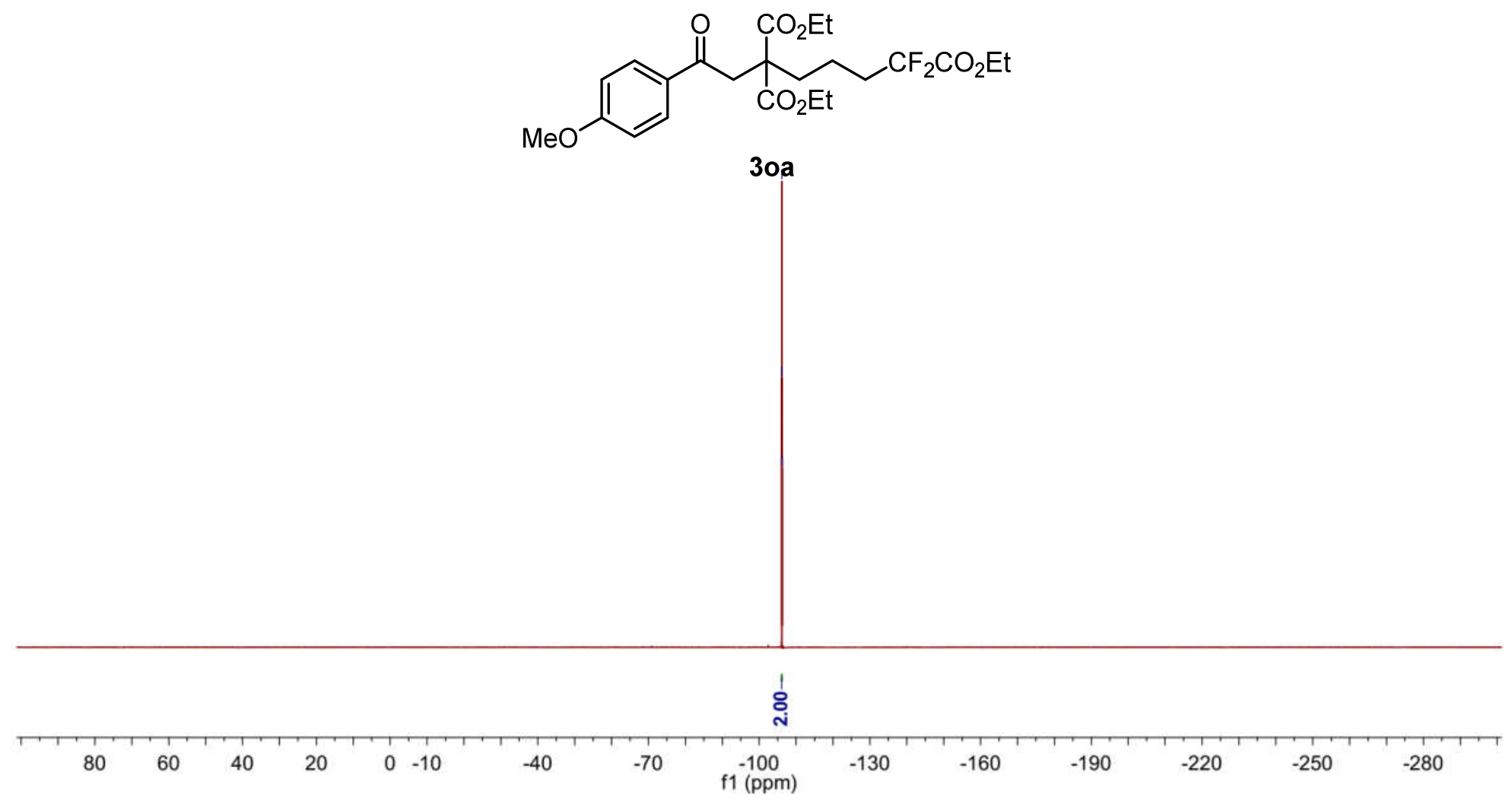




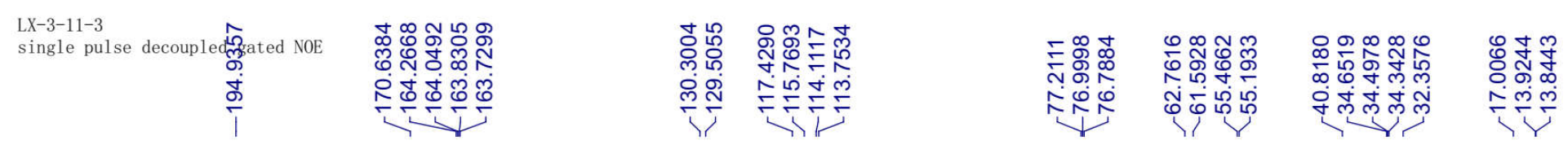
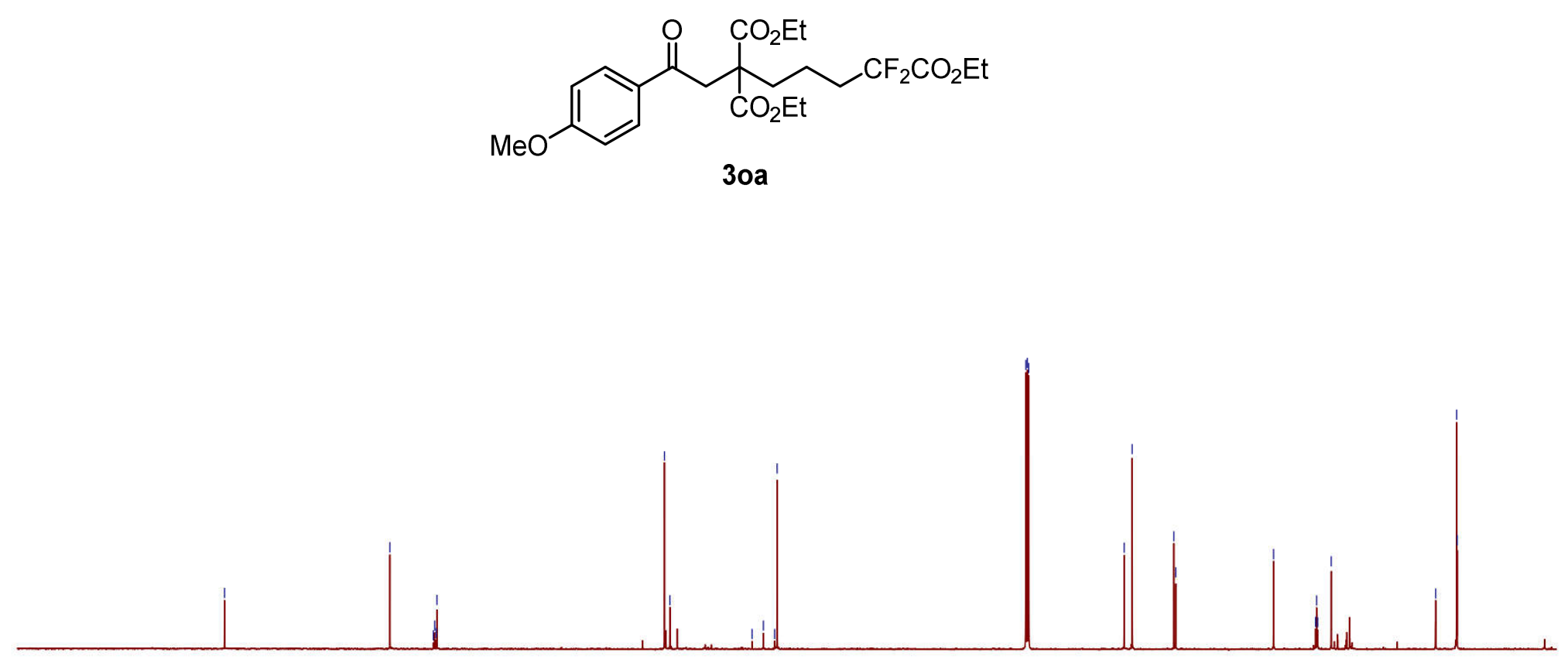

$\begin{array}{lllllllllllllllllllllll}220 & 210 & 200 & 190 & 180 & 170 & 160 & 150 & 140 & 130 & 120 & 110 & 100 & 90 & 80 & 70 & 60 & 50 & 40 & 30 & 20 & 10 & 0\end{array}$ 

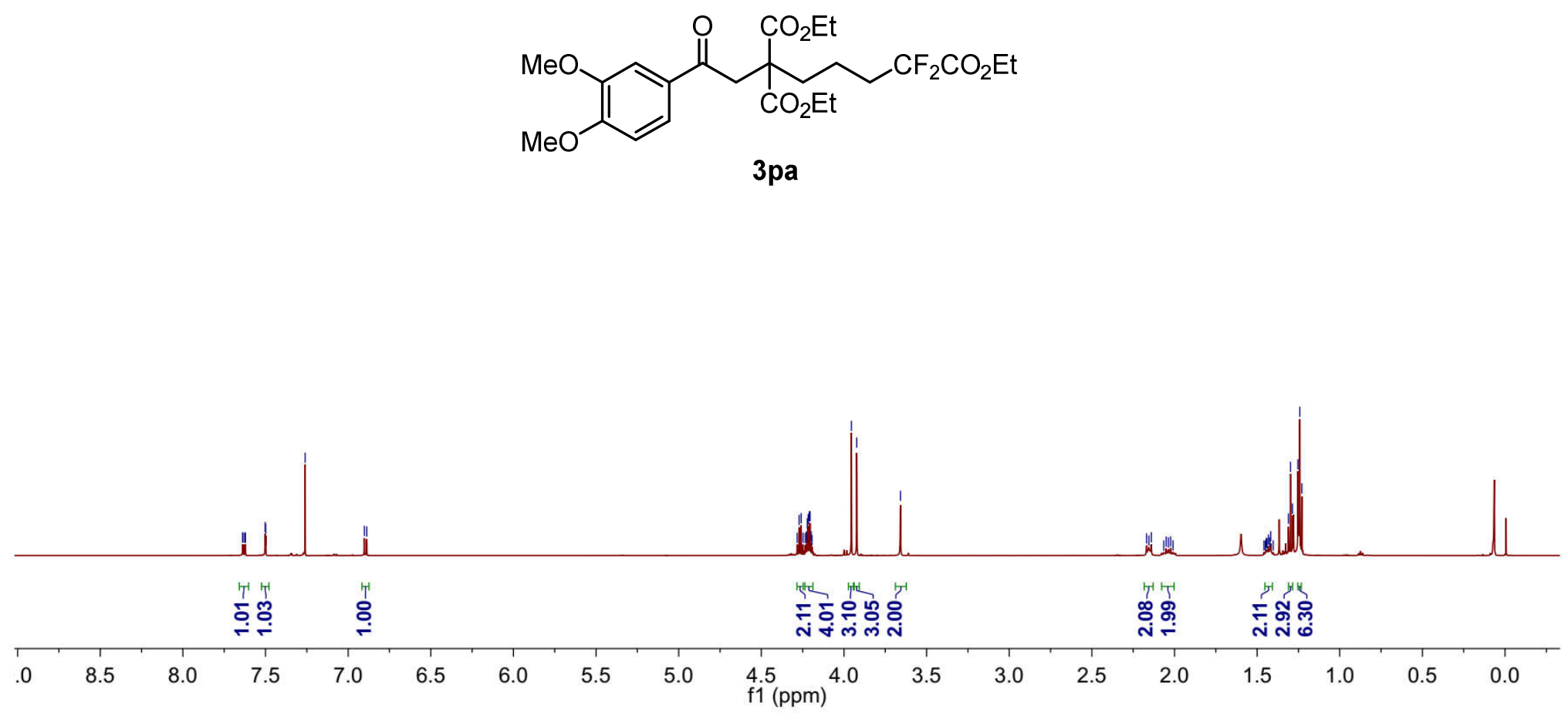
LX-3-11-5

single_pulse

:융유

윤유

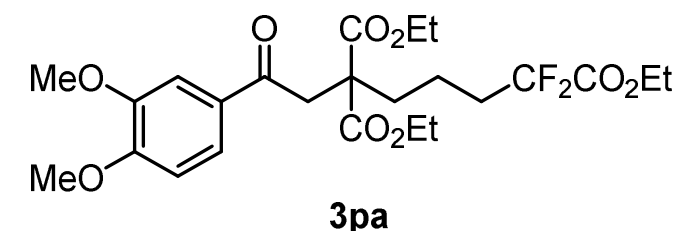

3 pa

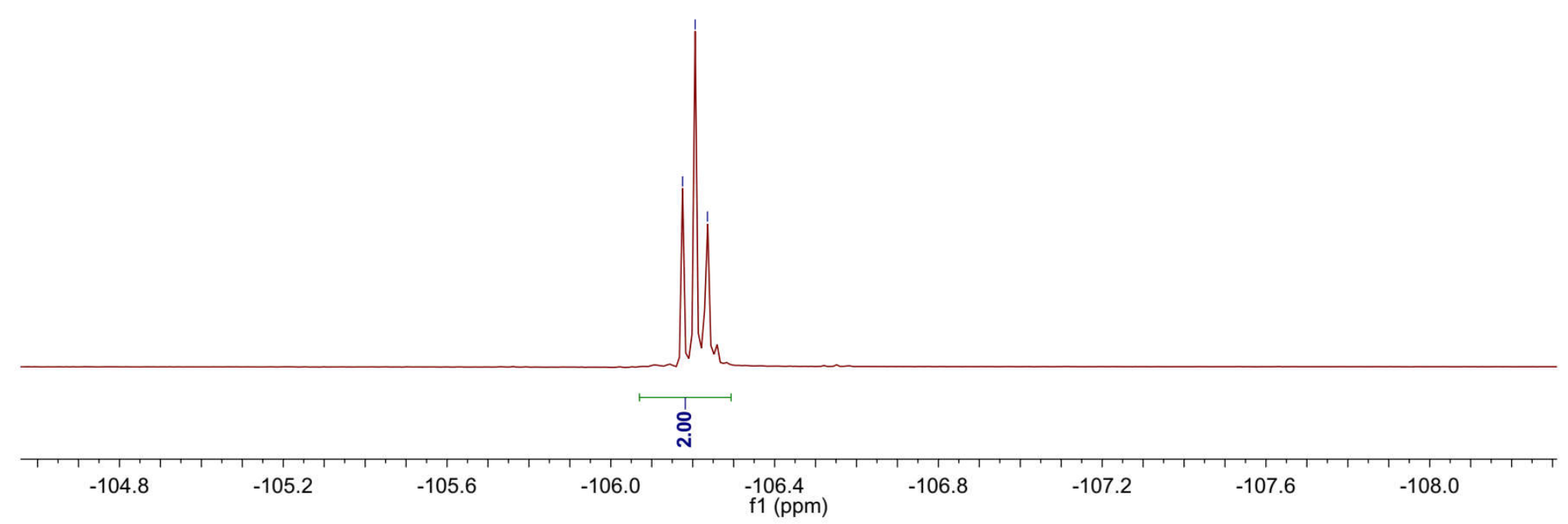

61 


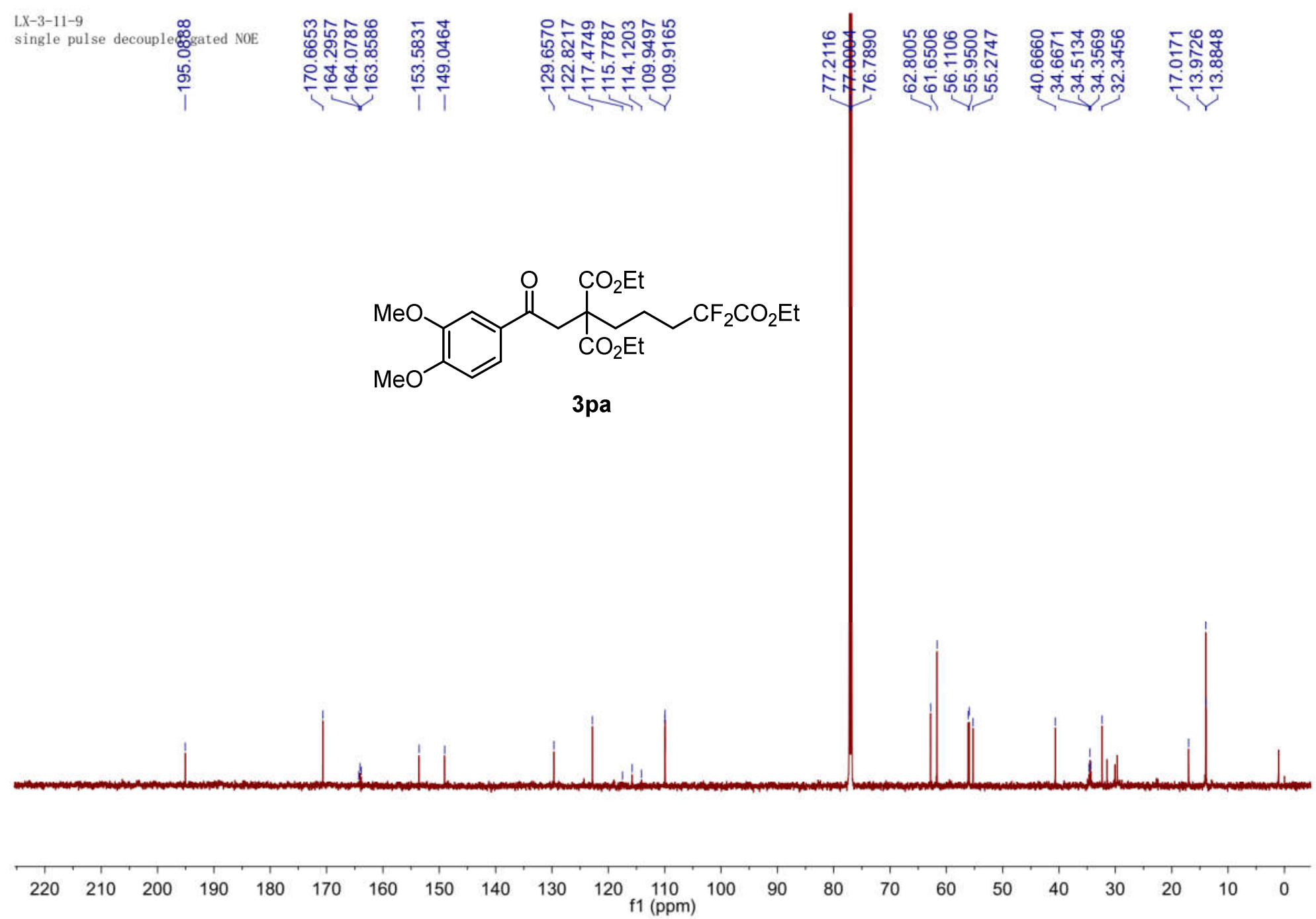



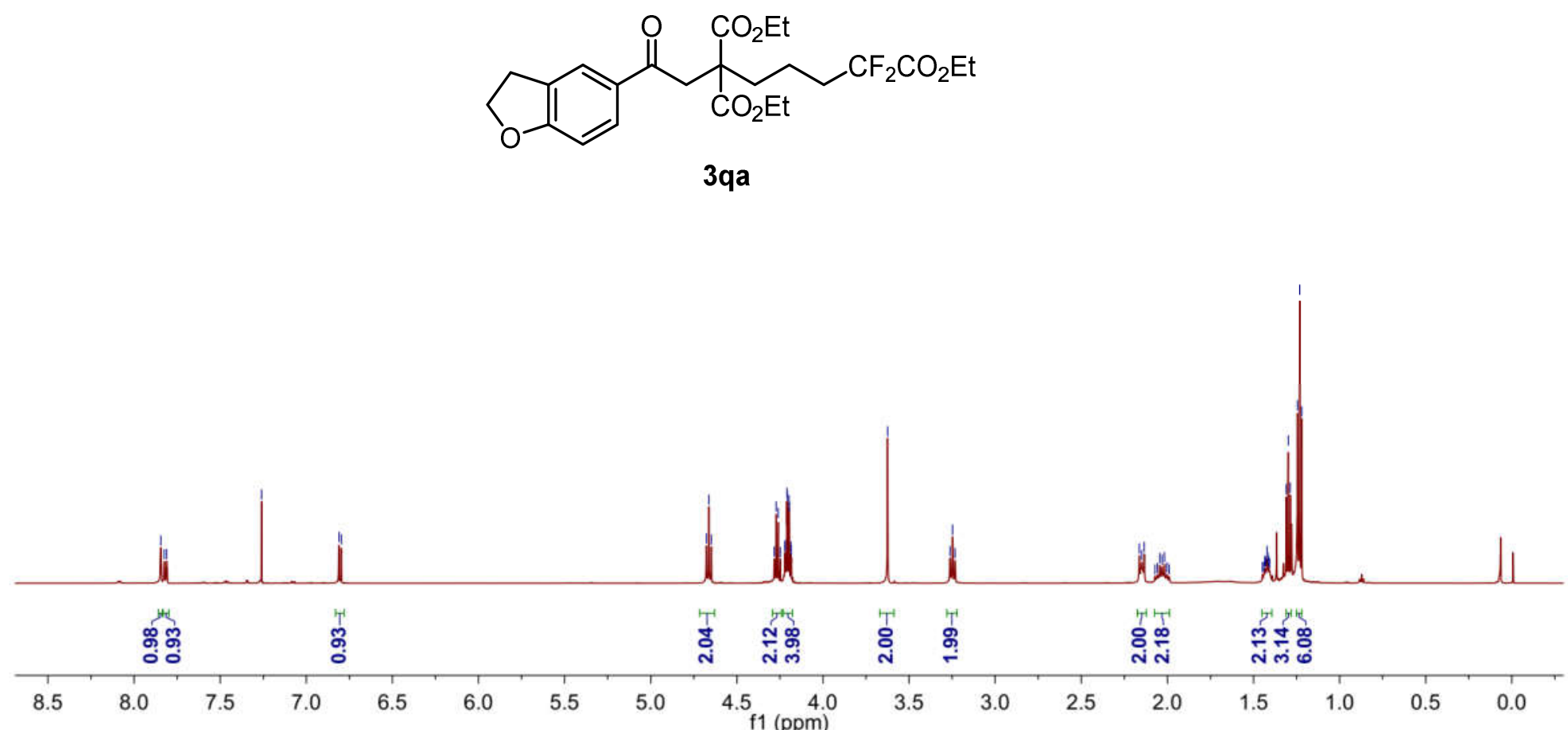


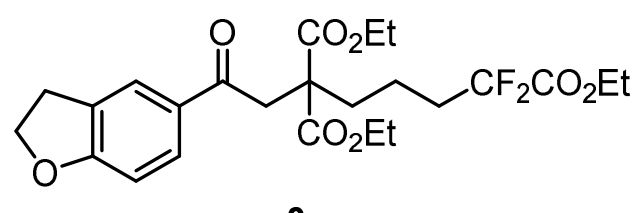

$3 q a$

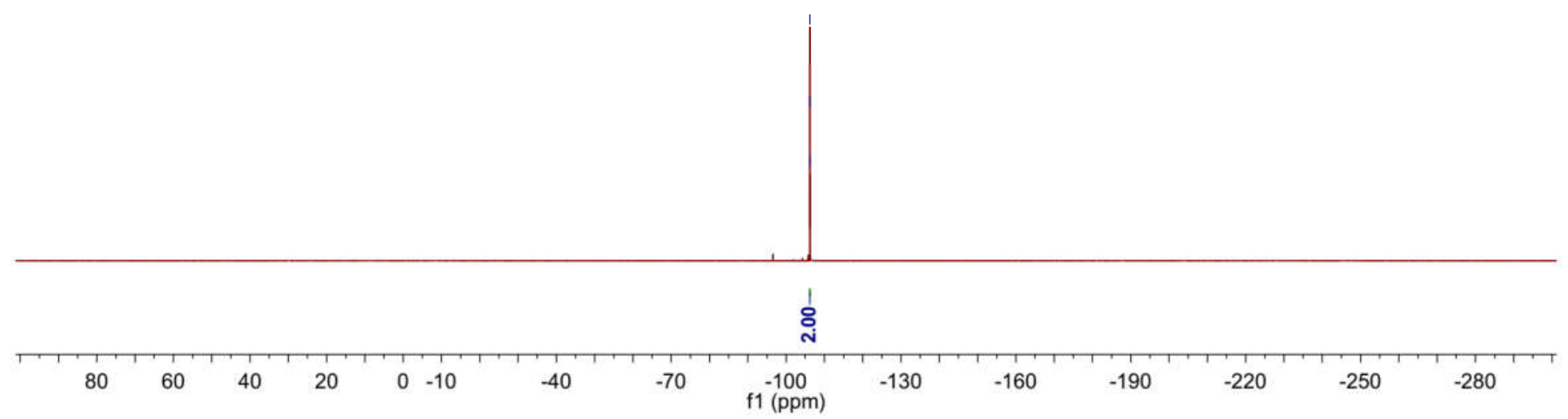




\begin{tabular}{|c|c|c|c|c|c|}
\hline 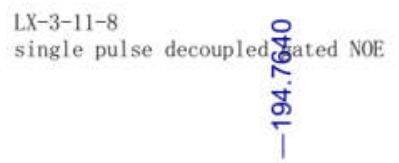 & 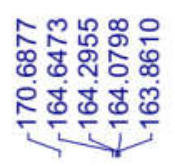 & 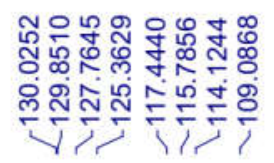 & 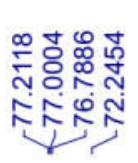 & 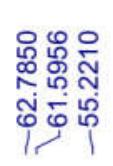 & 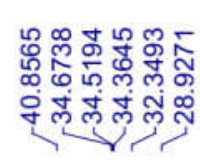 \\
\hline
\end{tabular}

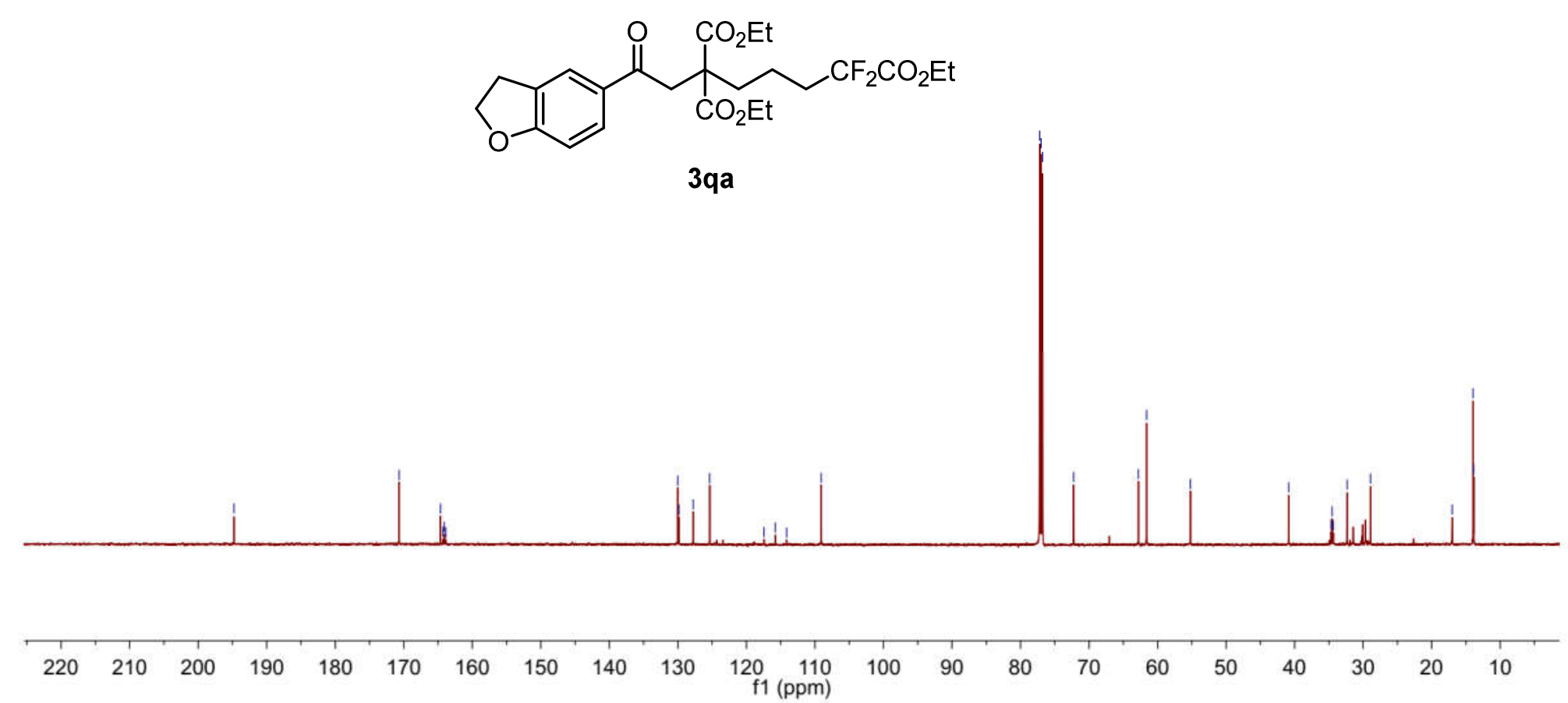




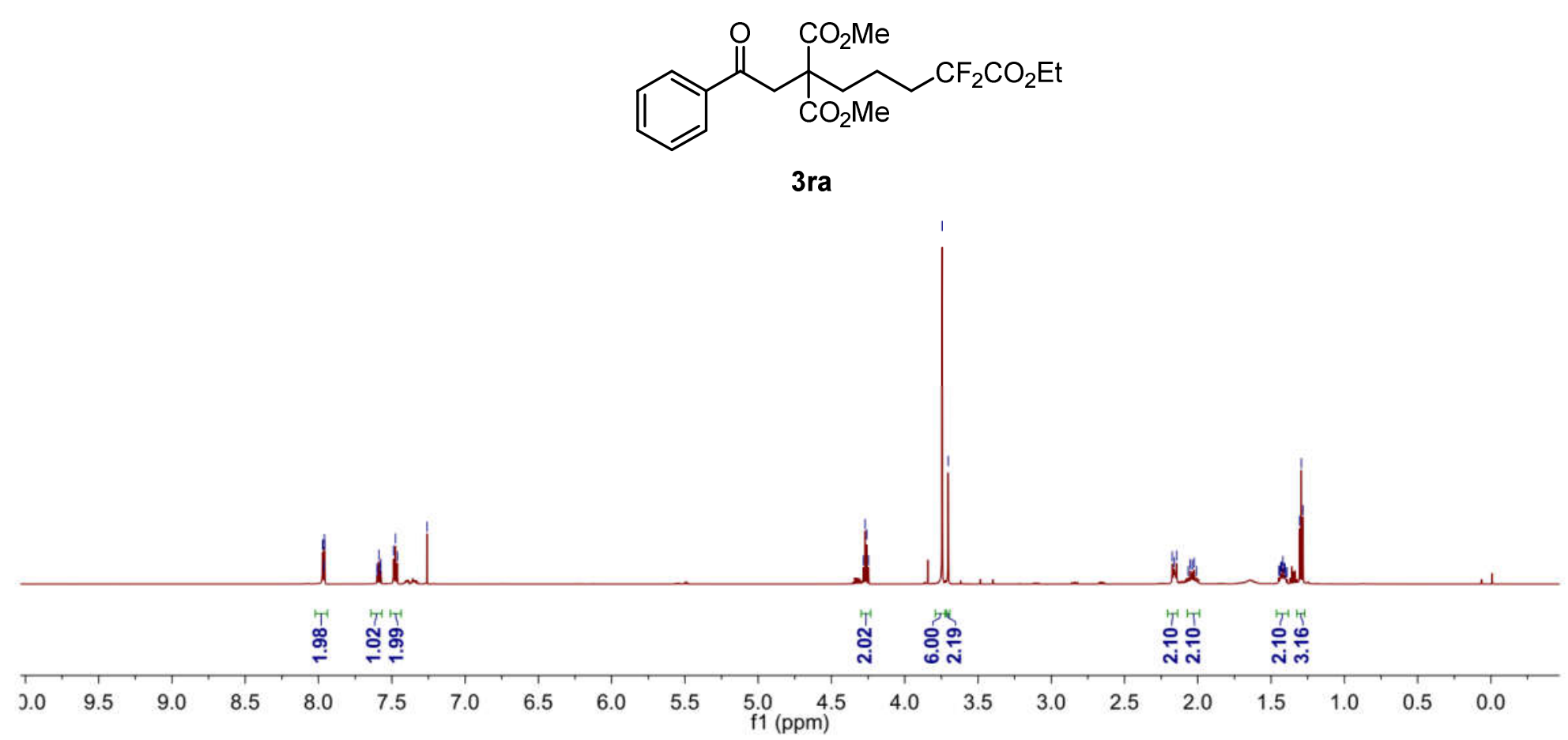




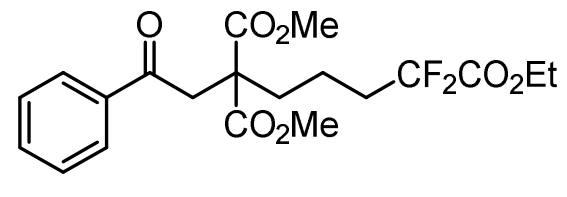

3ra

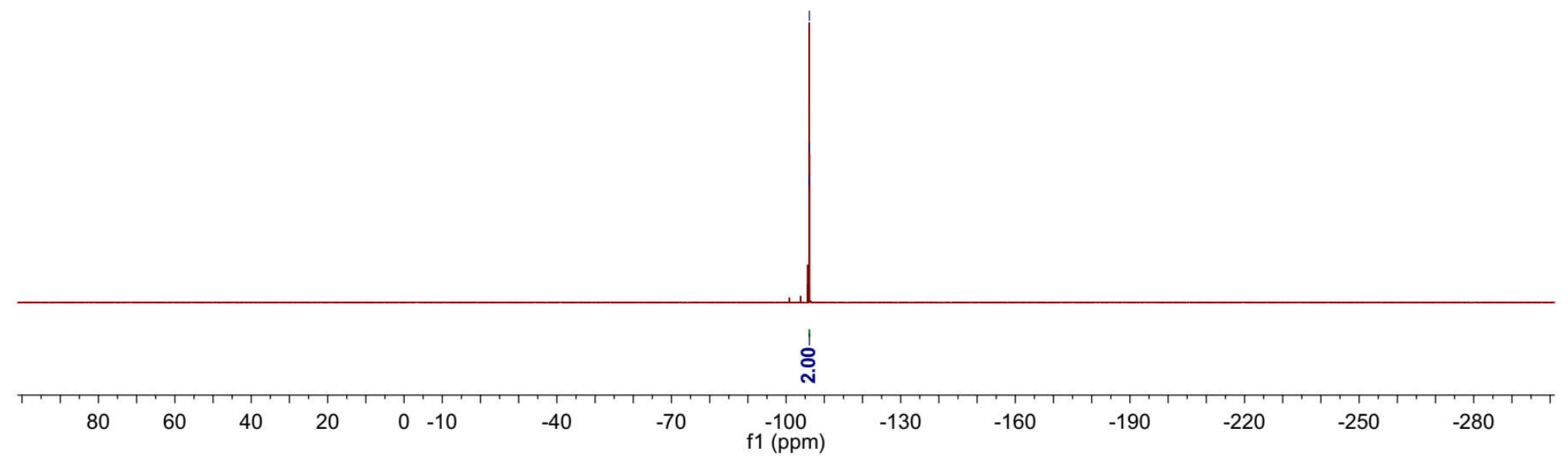




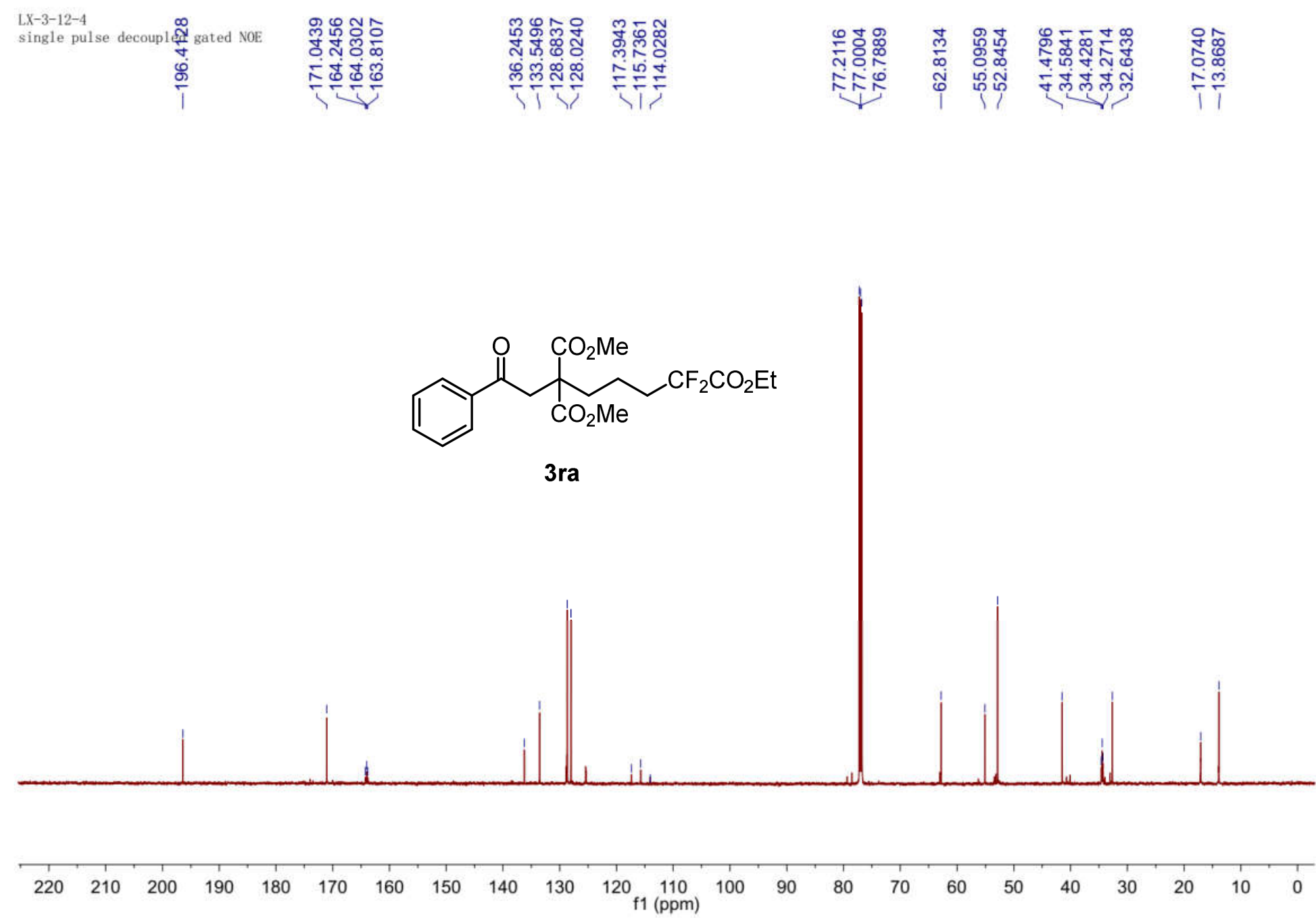




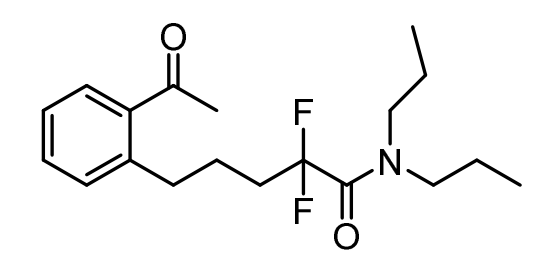

$3 a b$

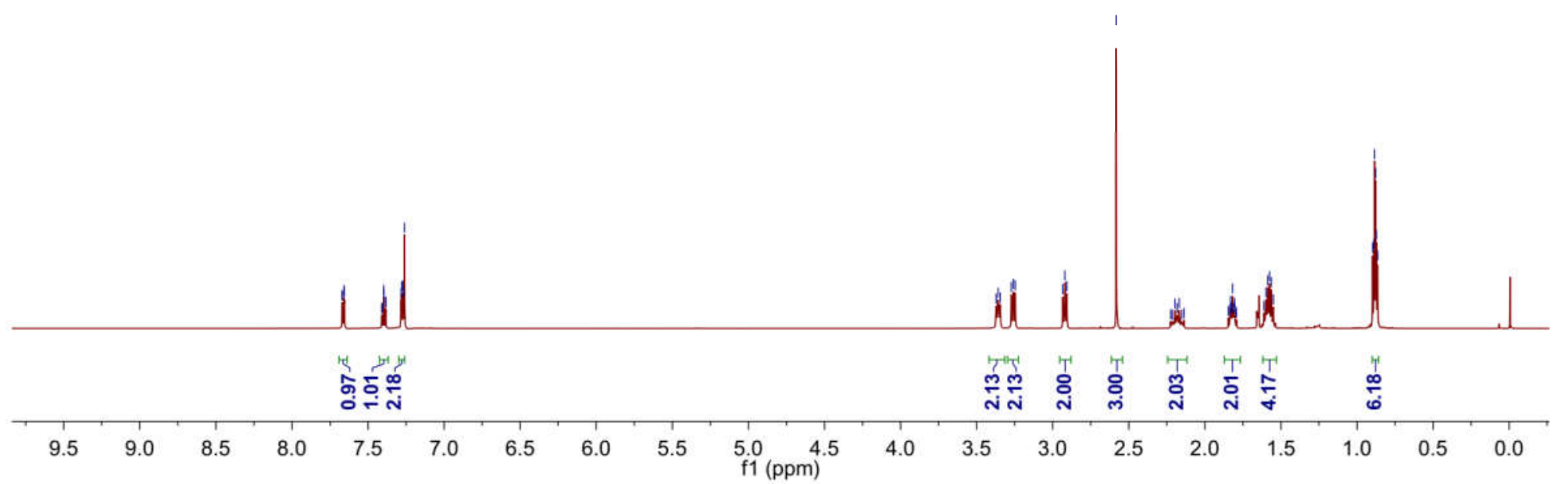




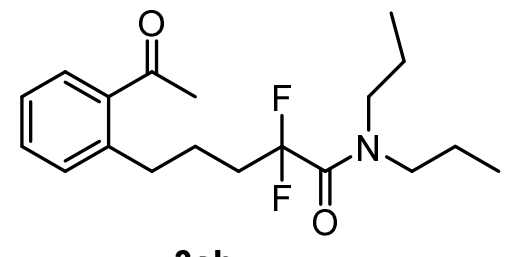

$3 a b$

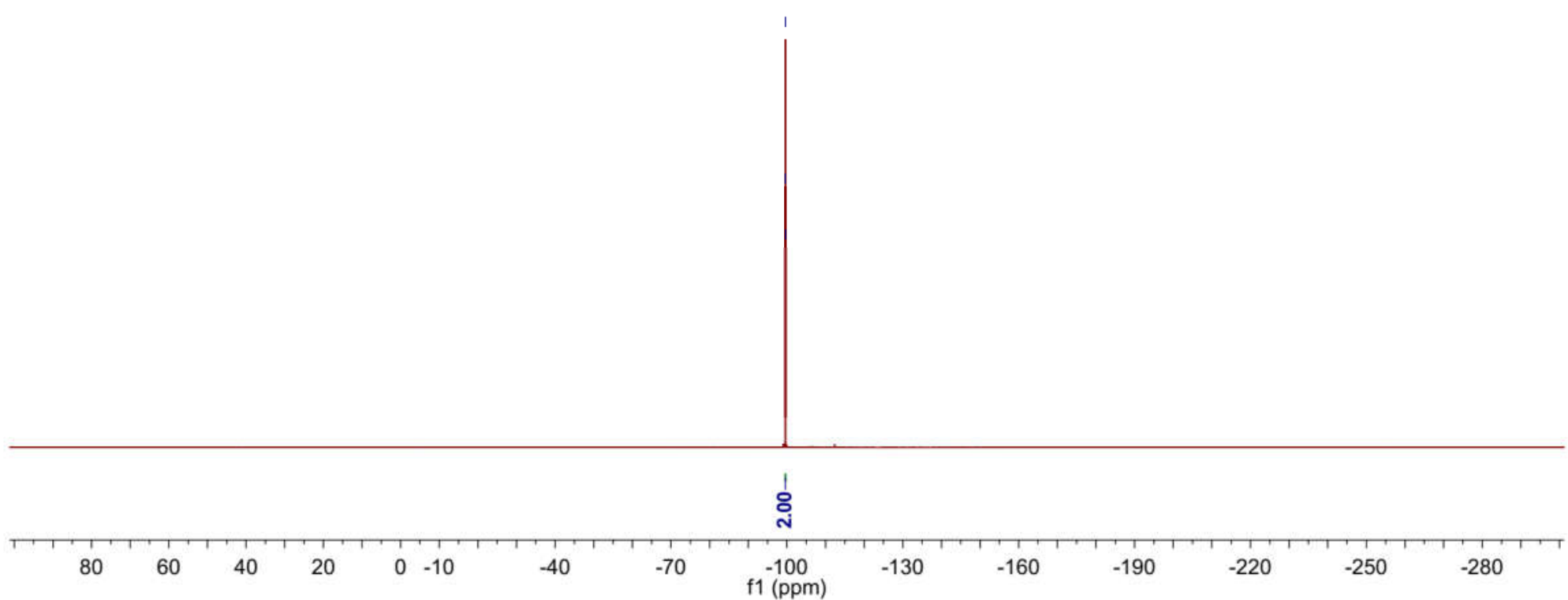




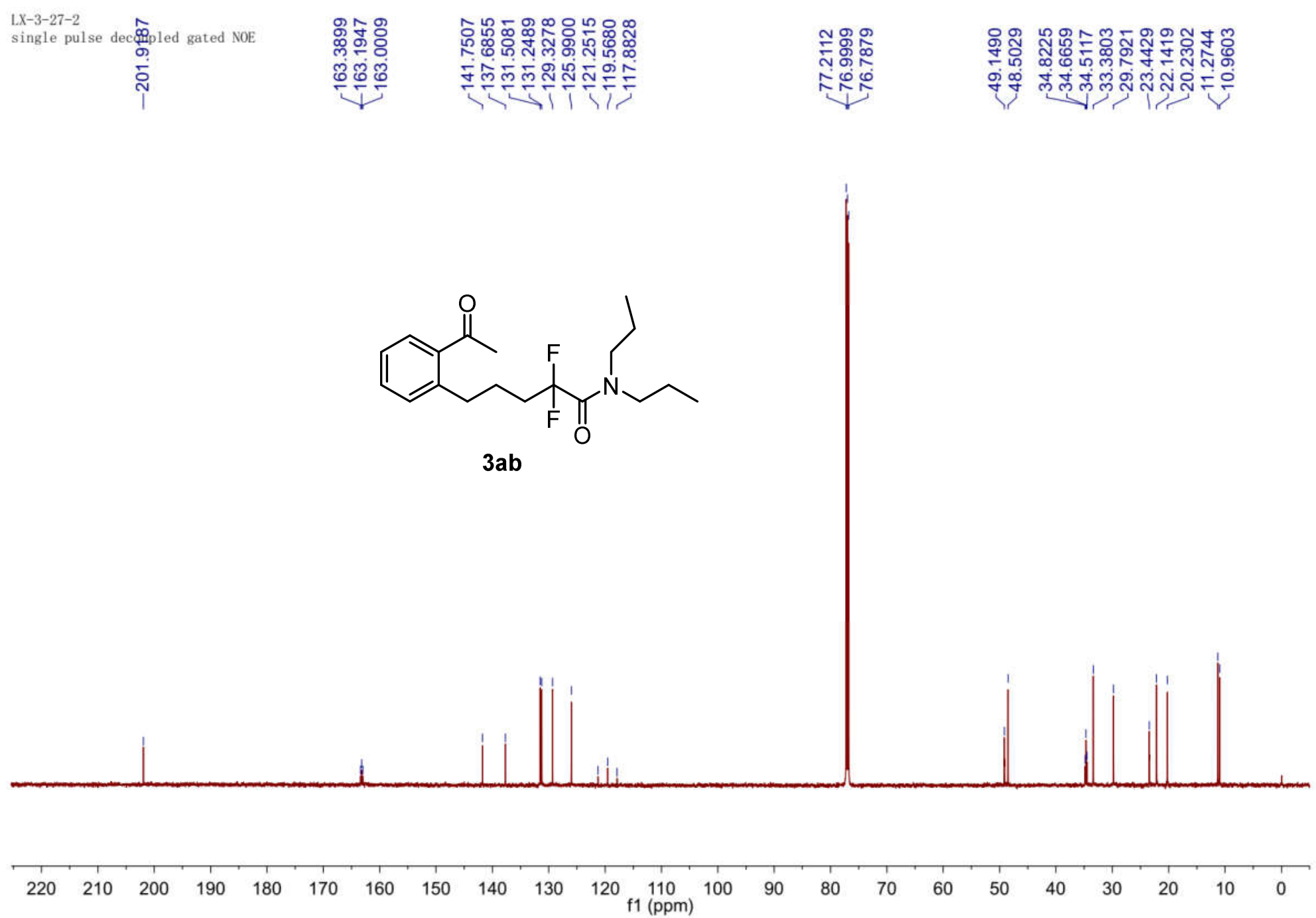




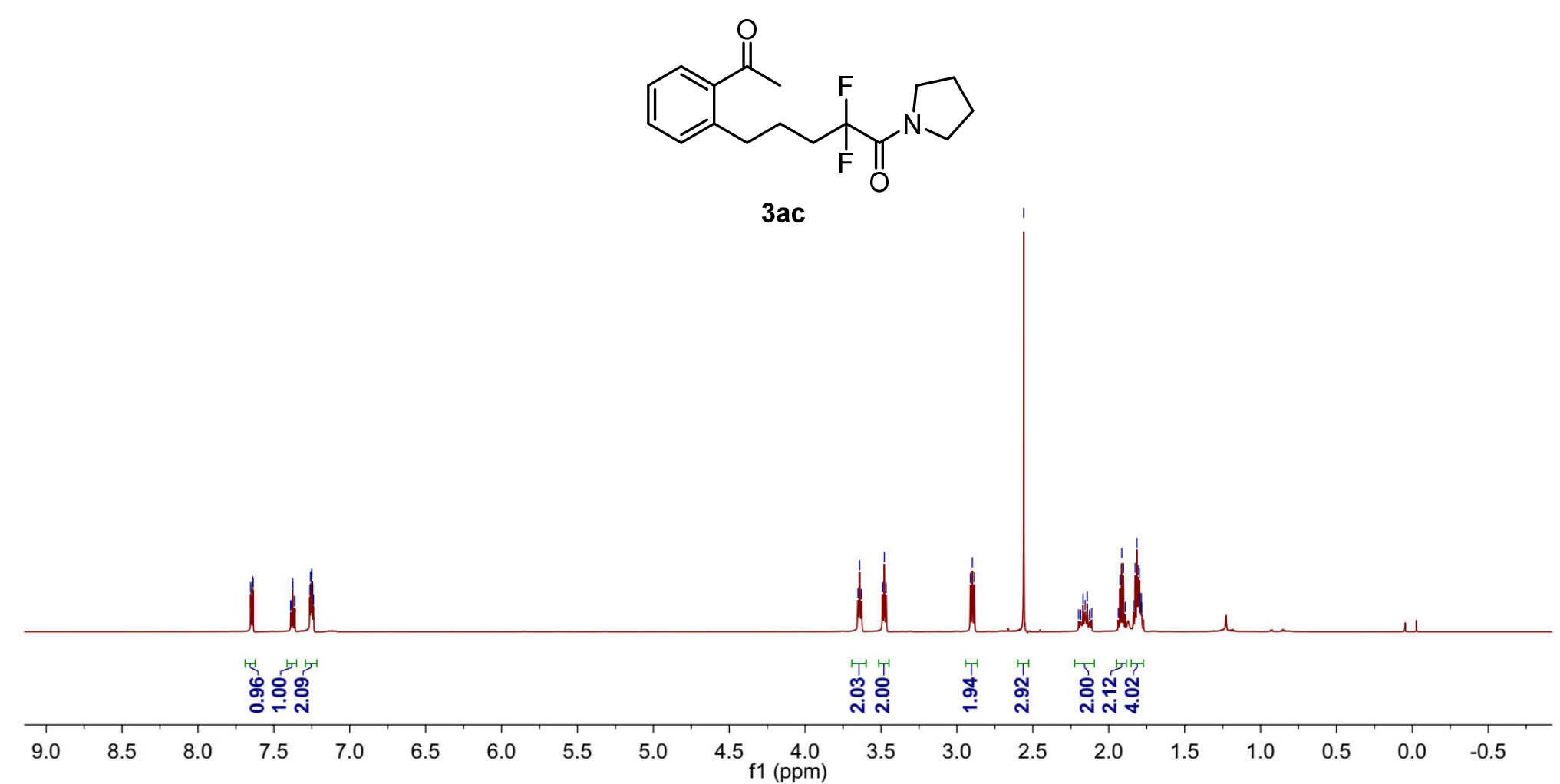


政

然题

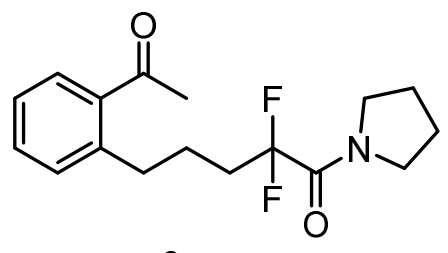

$3 a c$

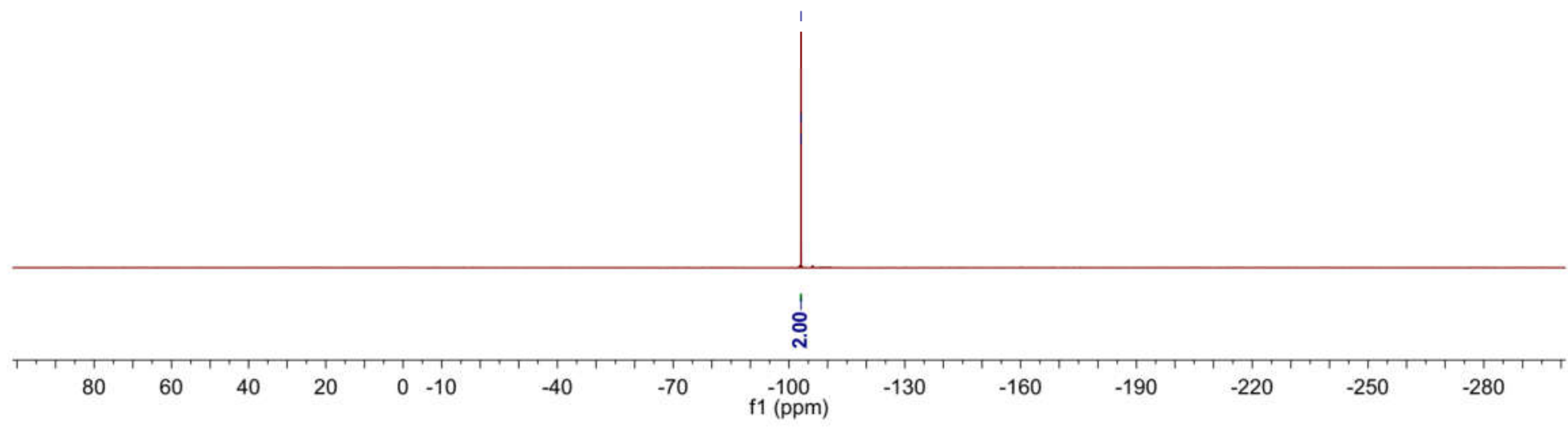




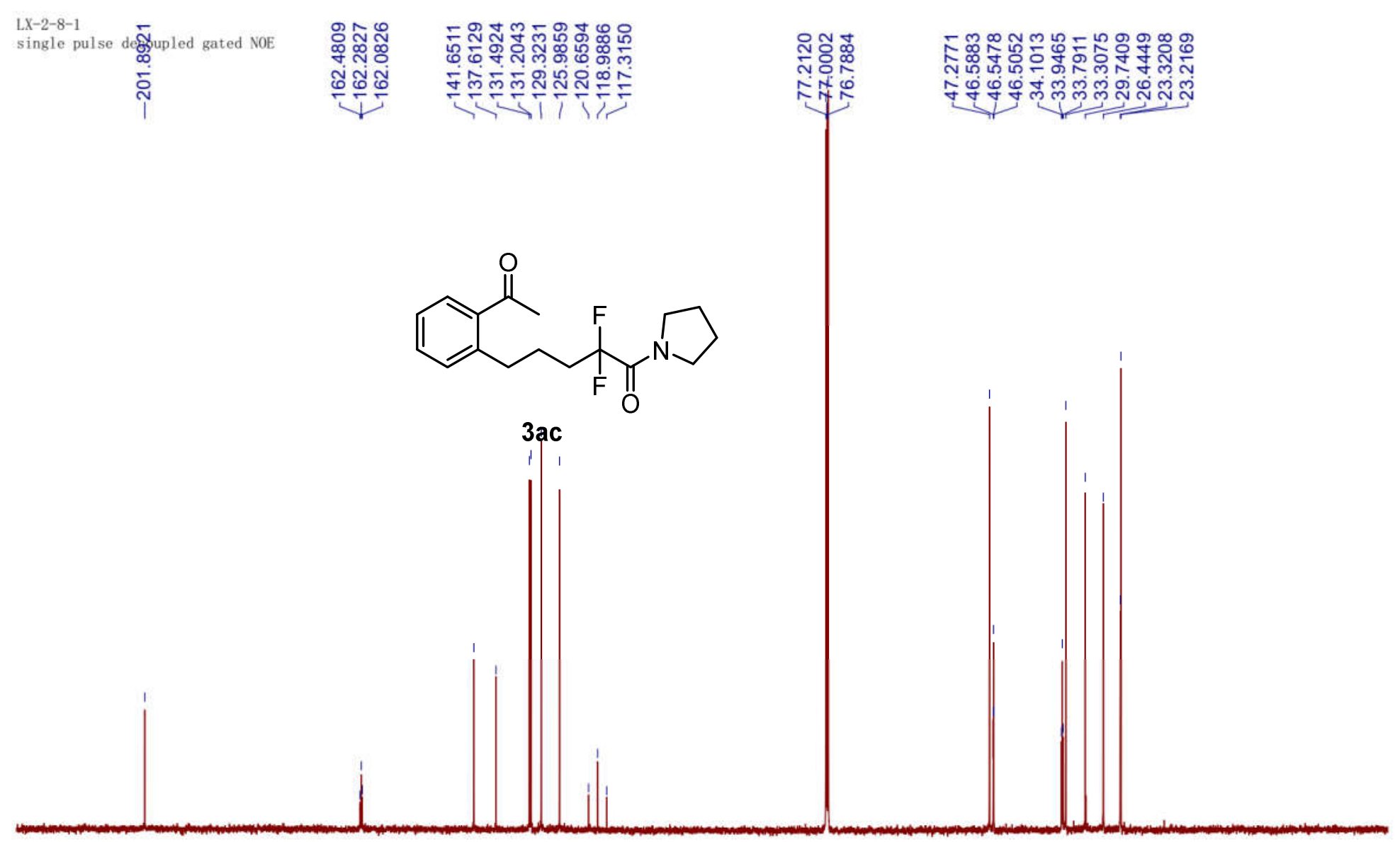

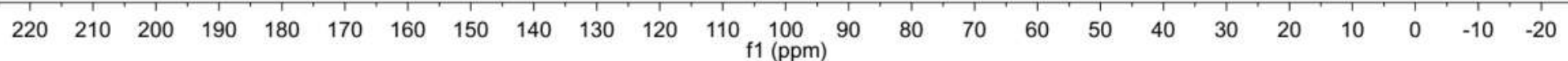




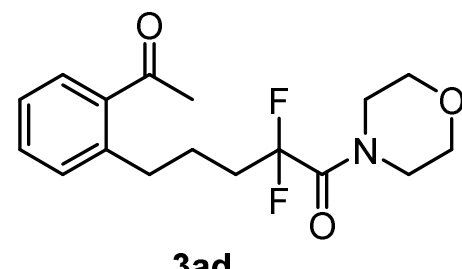

$3 a d$

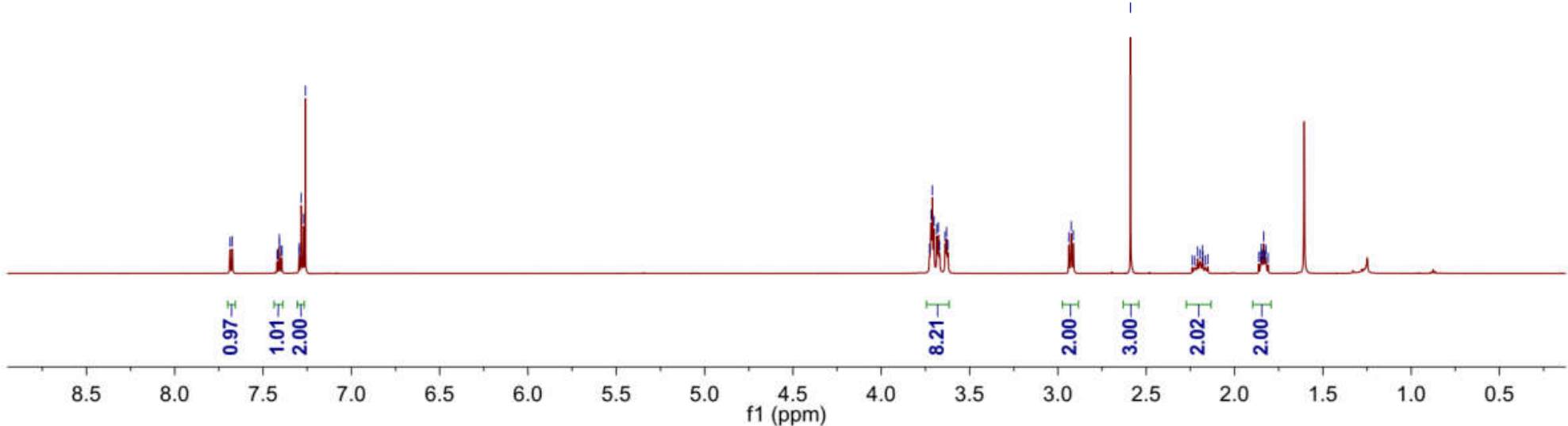


LX-3-27-3

single_puls

동ㅎㅀ음

केक्ष

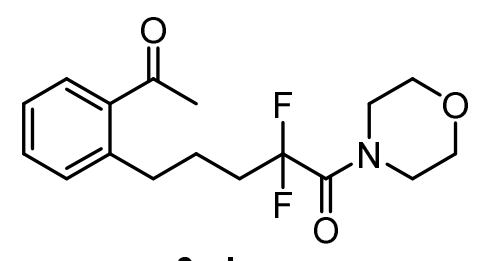

$3 \mathrm{ad}$

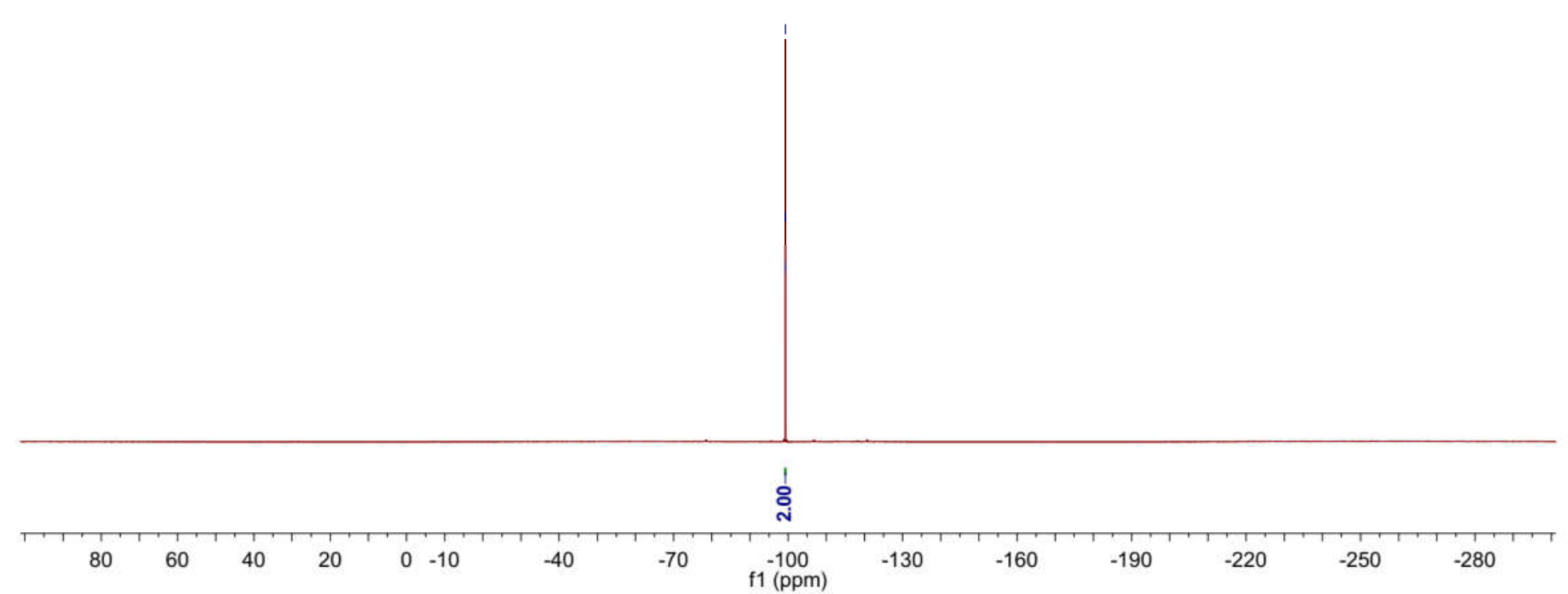



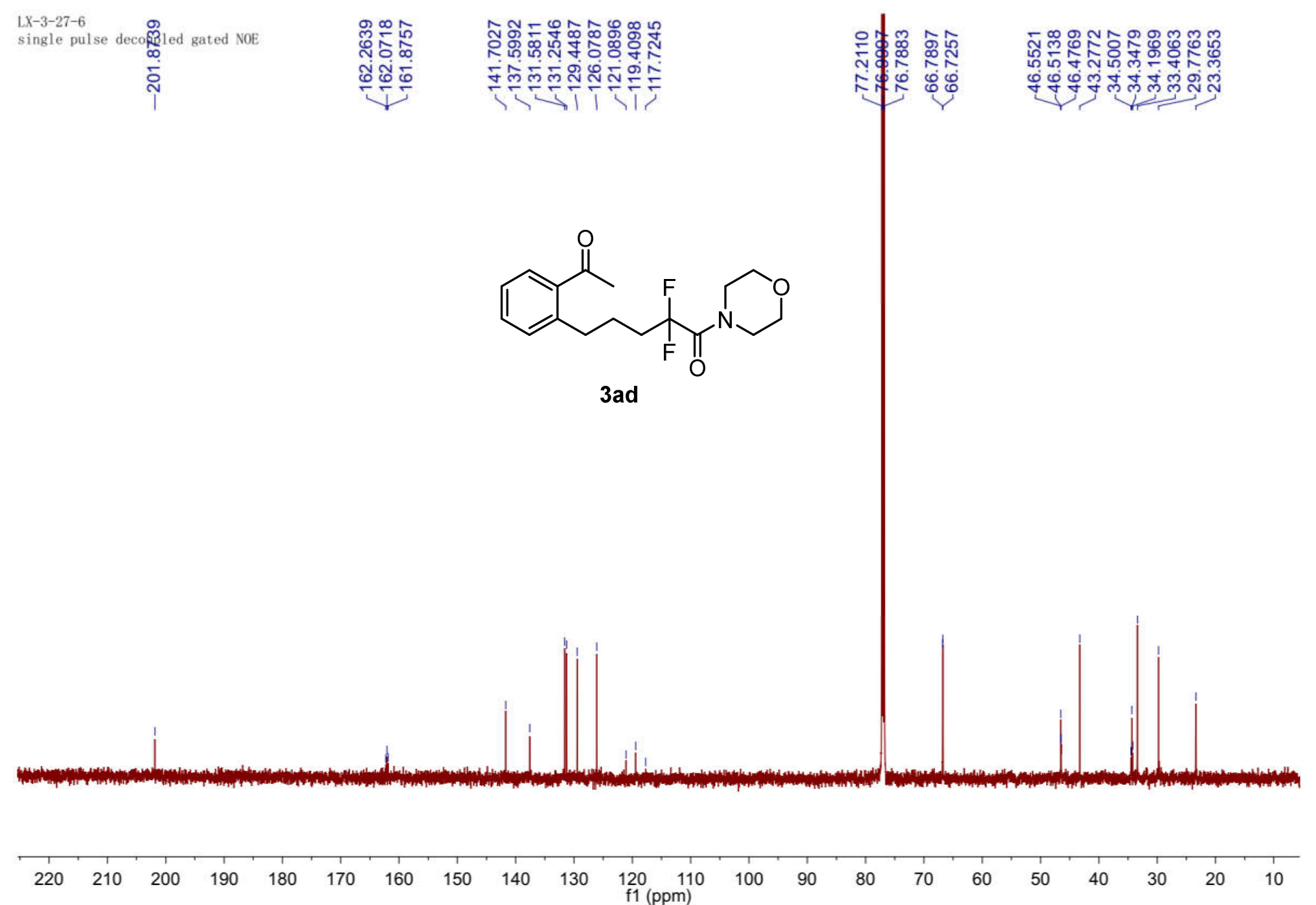


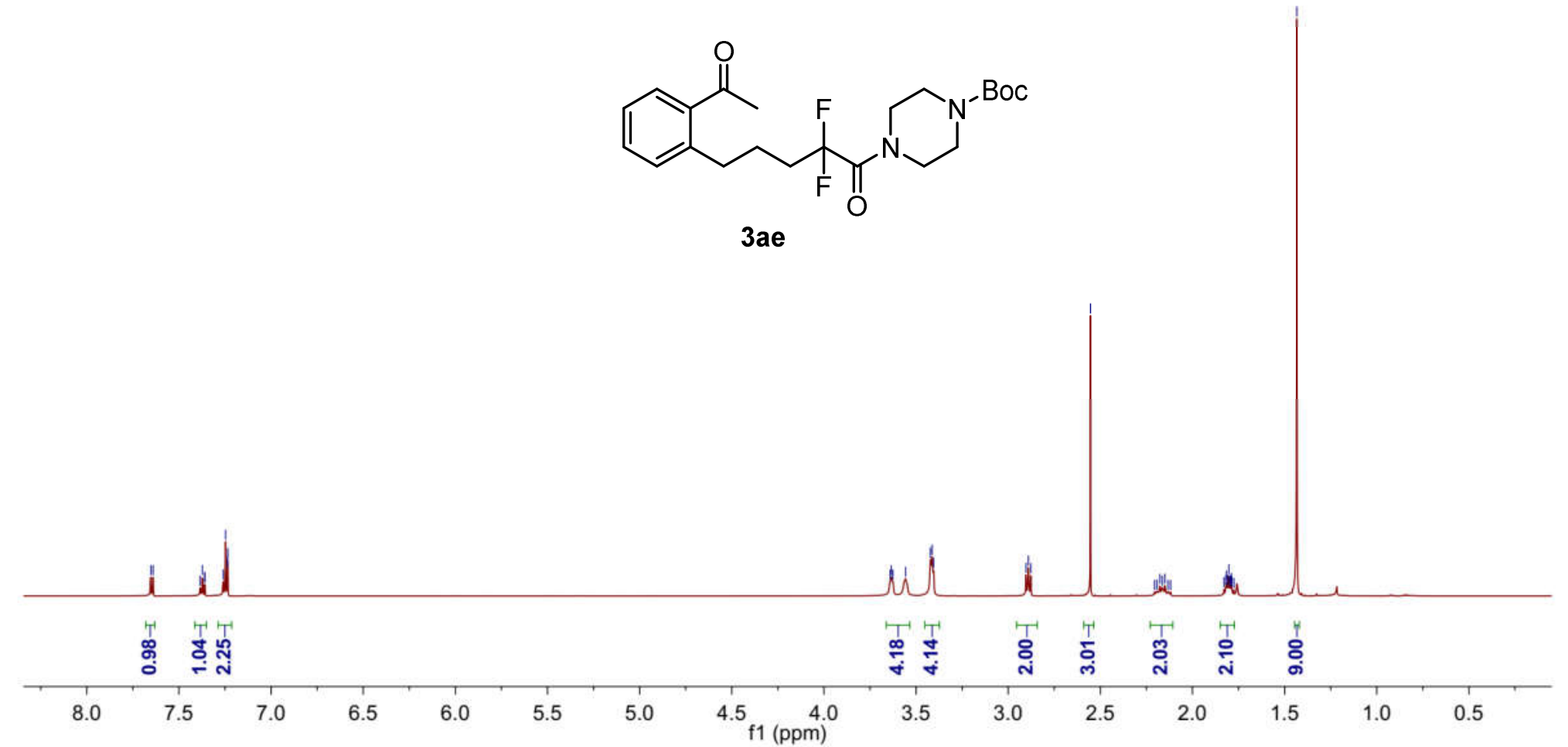


LX-3-27-4

single_pulse

ธ్ర

i্

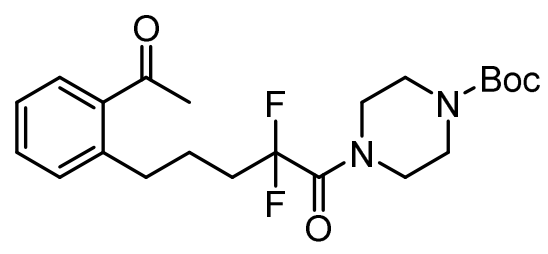

$3 a e$

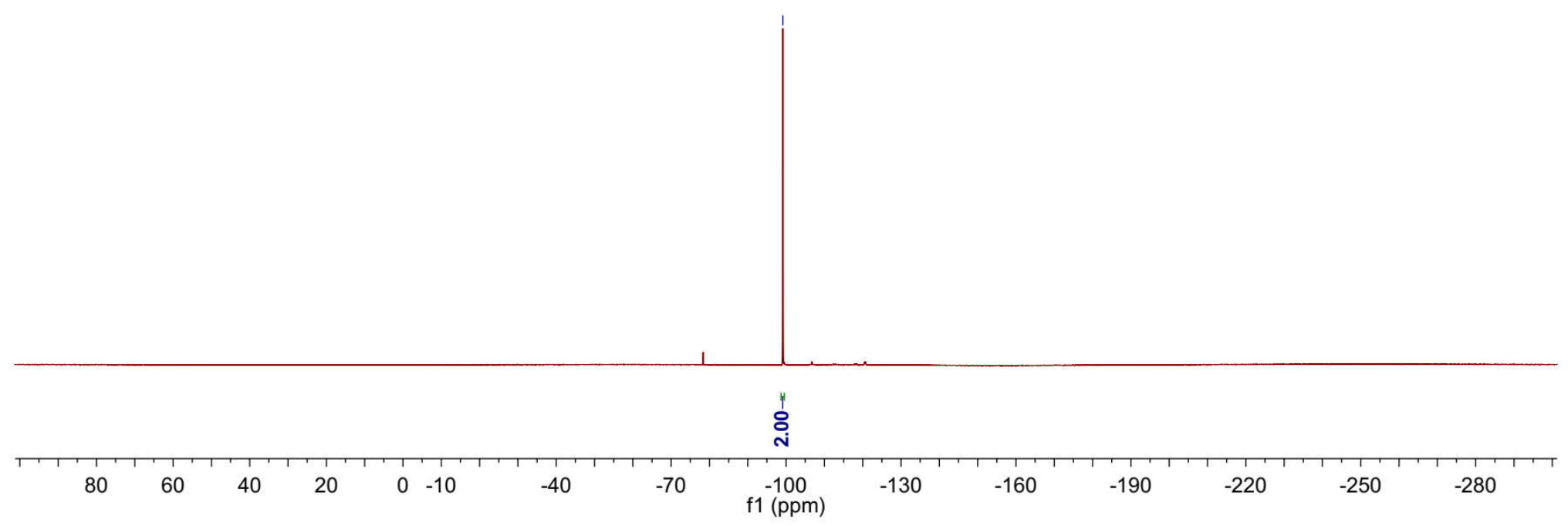

79 

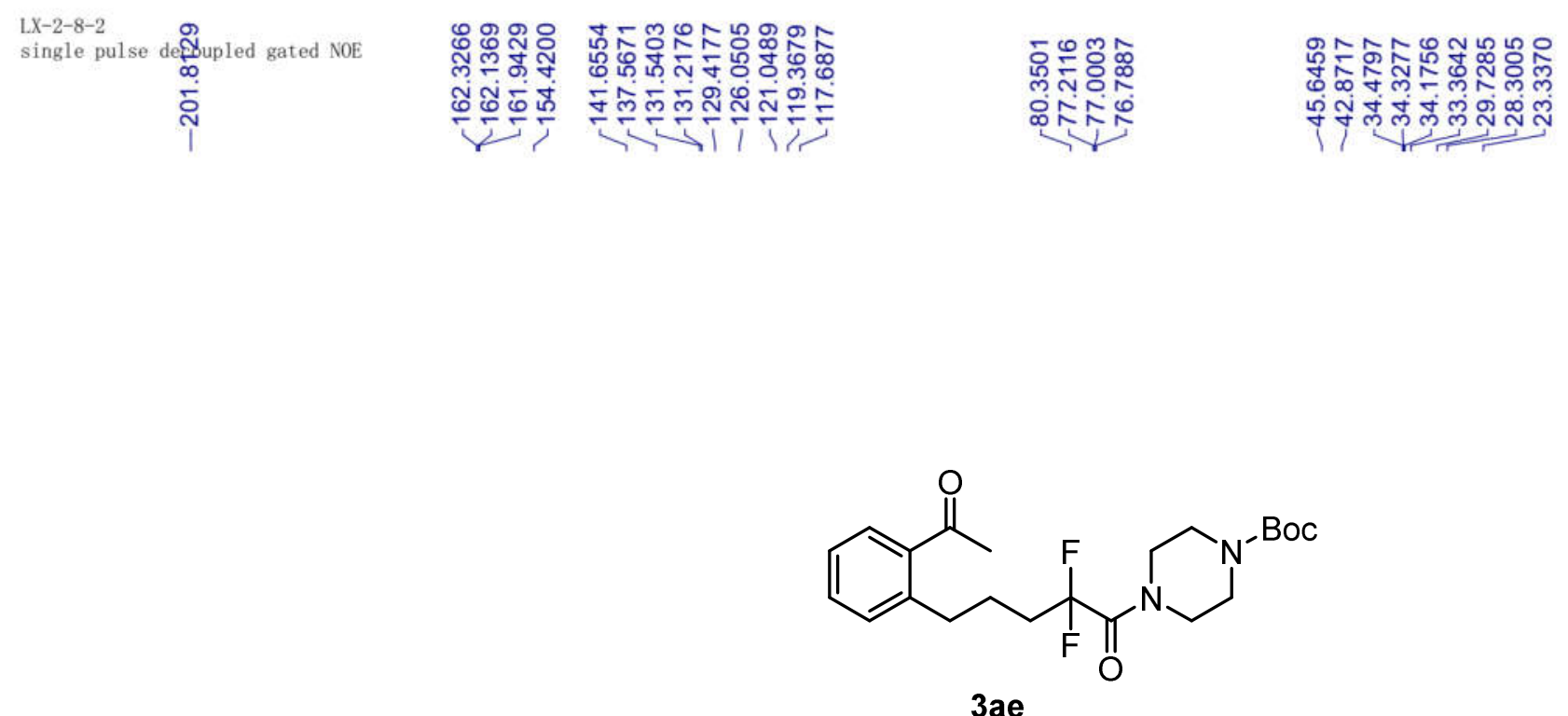

$3 a e$

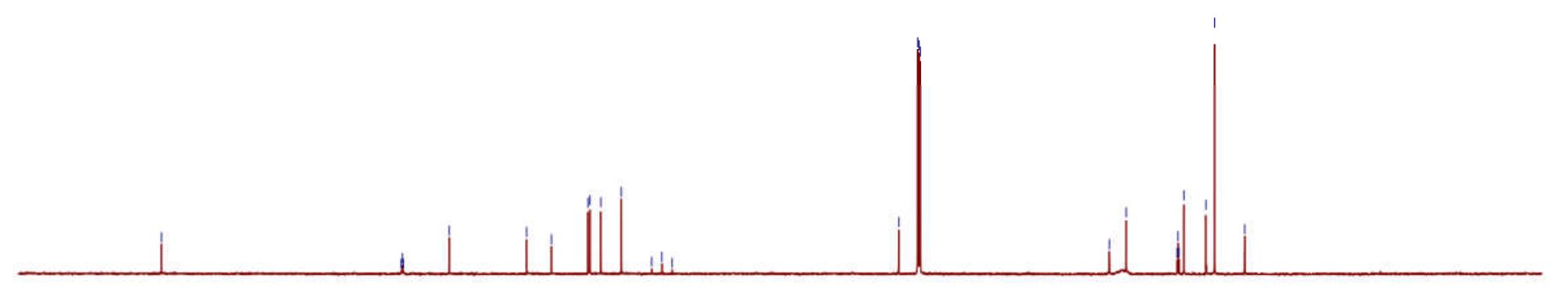

$\begin{array}{lllllllllllllllllllllllllllllllllllll}220 & 210 & 200 & 190 & 180 & 170 & 160 & 150 & 140 & 130 & 120 & 110 & 100 & 90 & 80 & 70 & 60 & 50 & 40 & 30 & 20 & 10 & 0 & -10 & -20\end{array}$ 
LX-3-17-6

single_pulse

ㄷํㅇำㅇำ

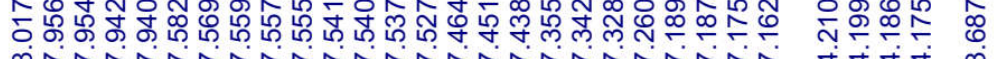

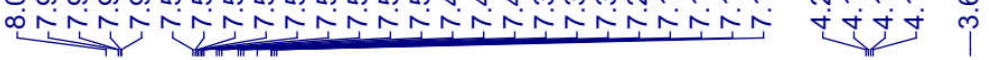

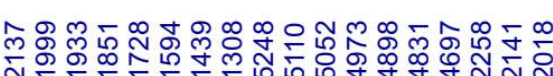

年

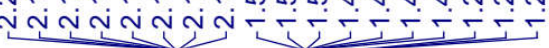

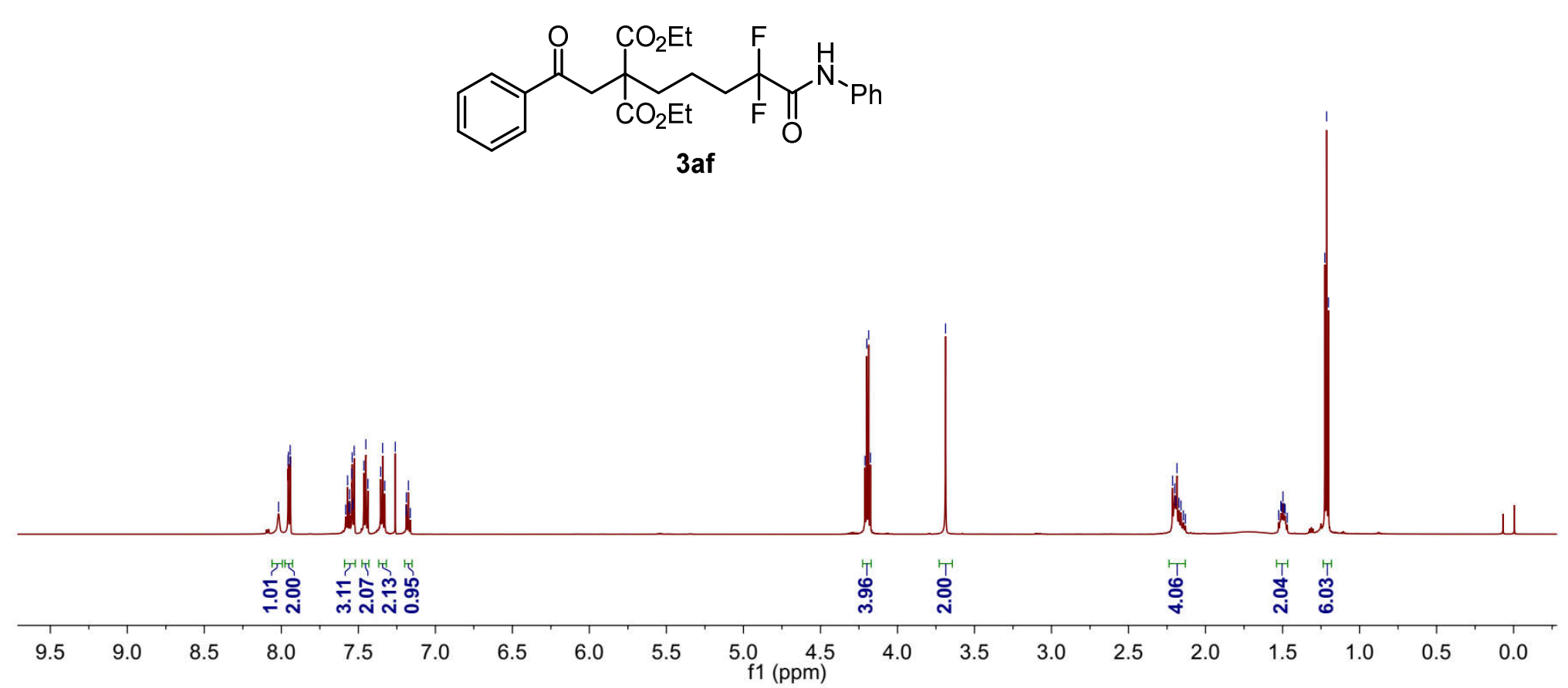

81 
LX-3-17-6

single_pulse
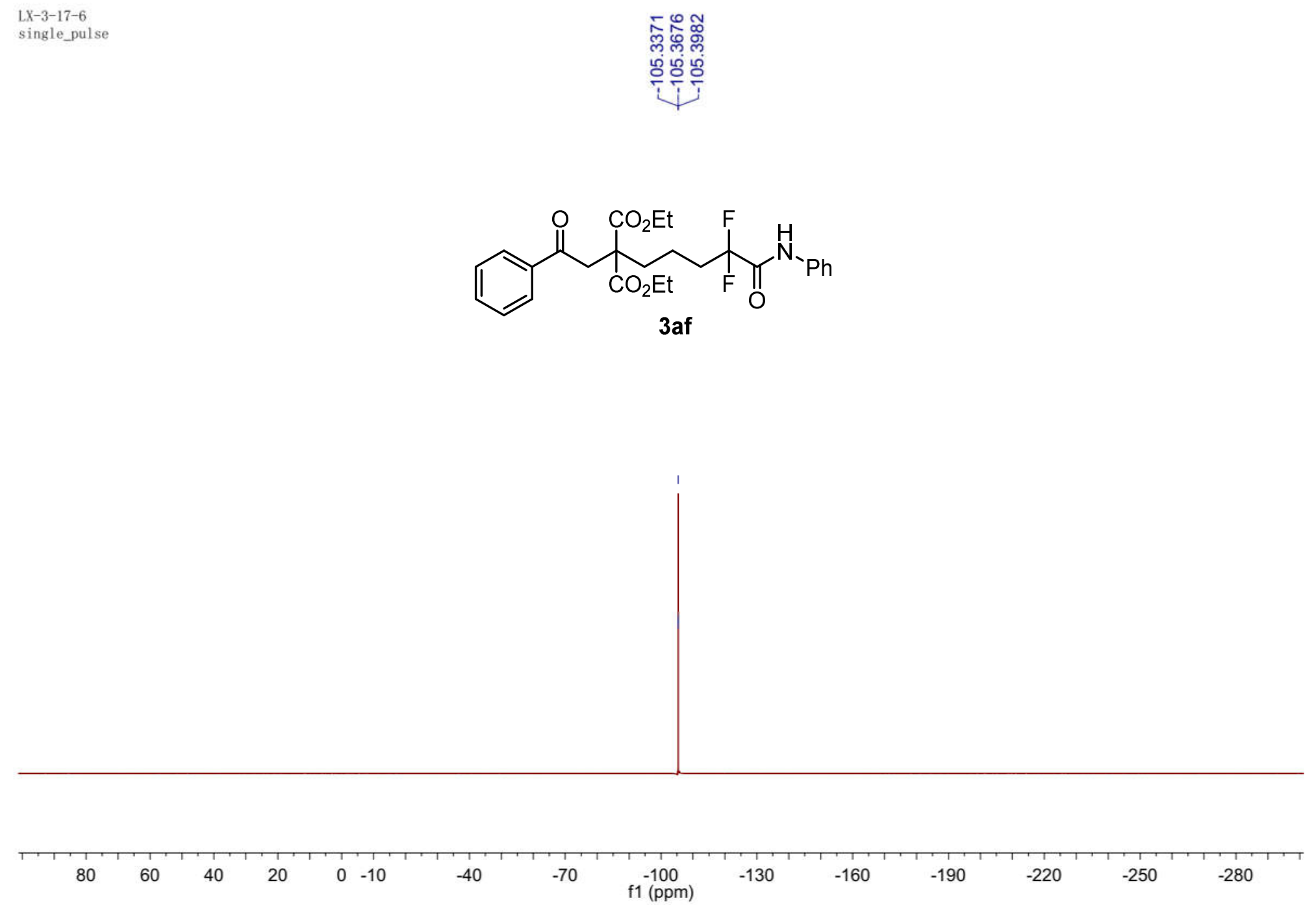


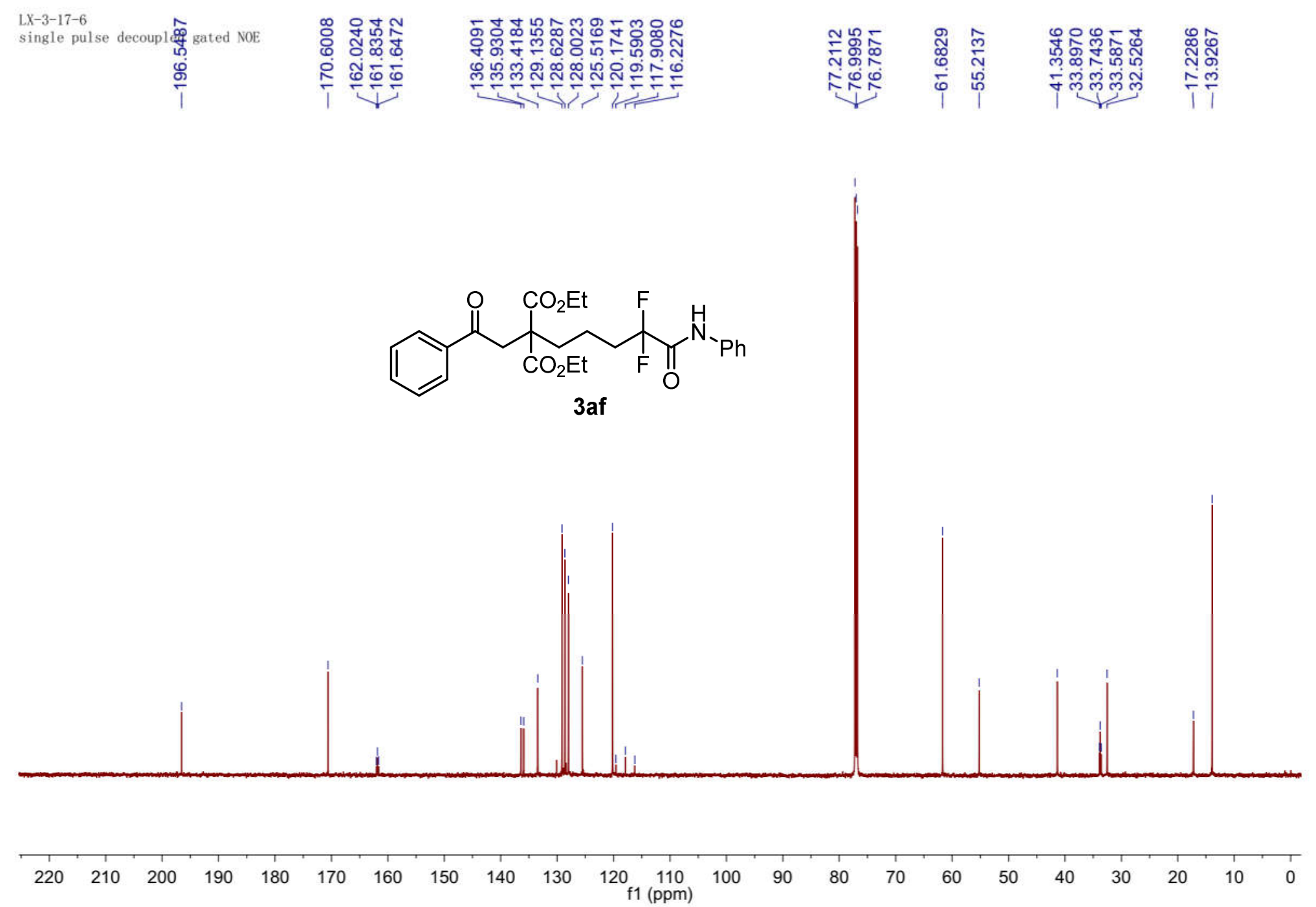




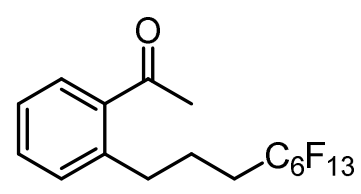

3ag

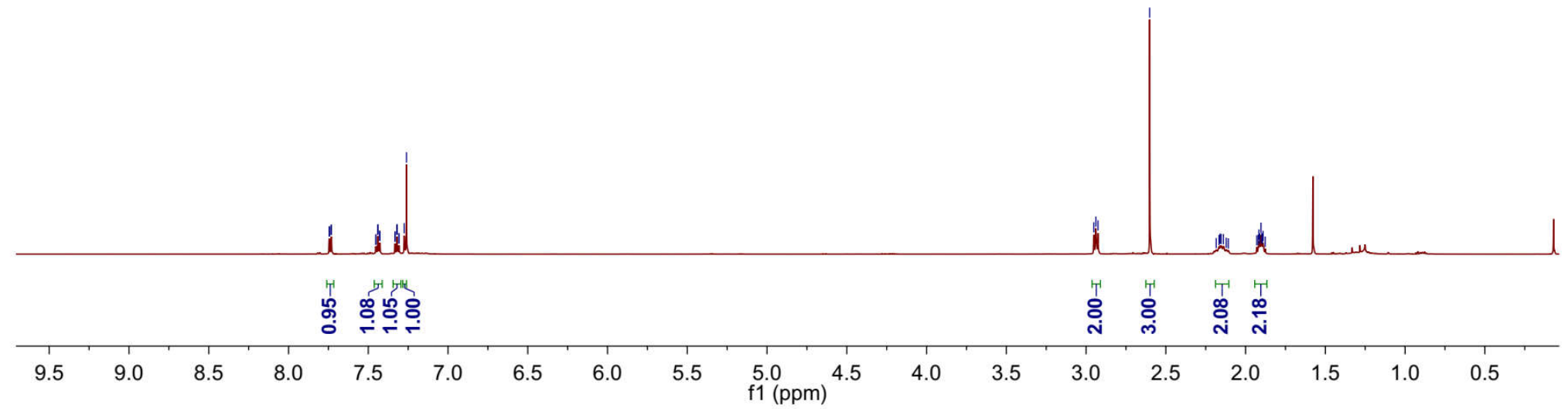


LX-3-27-5

single_pulse

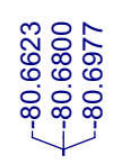

워요요

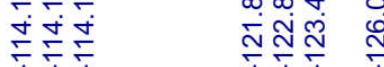

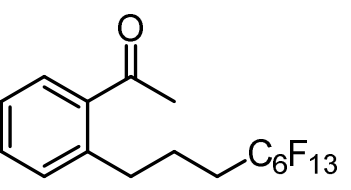

3ag

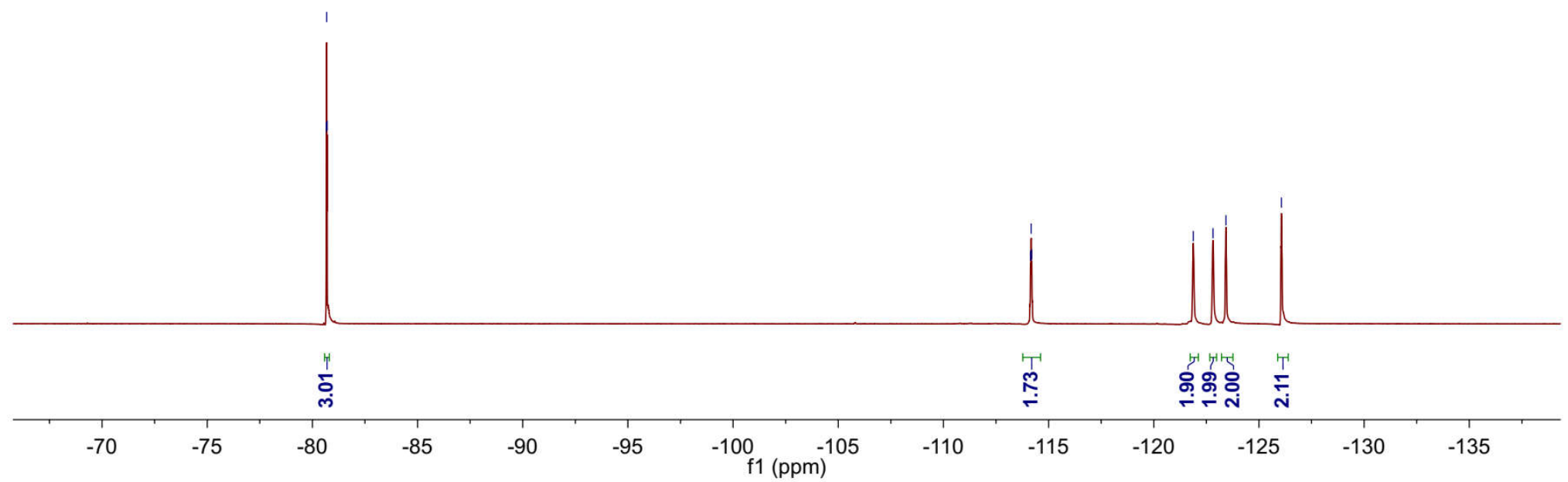




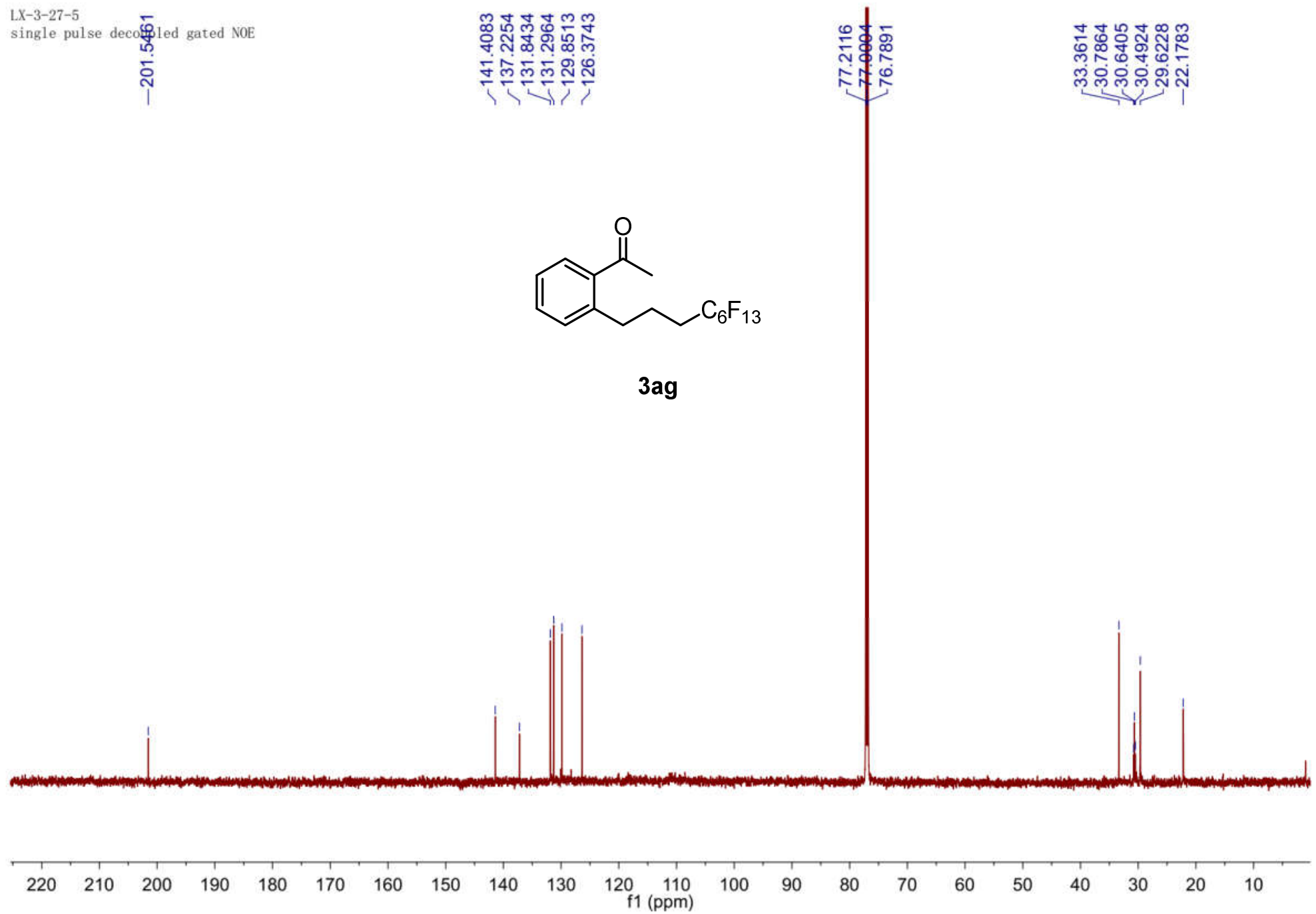




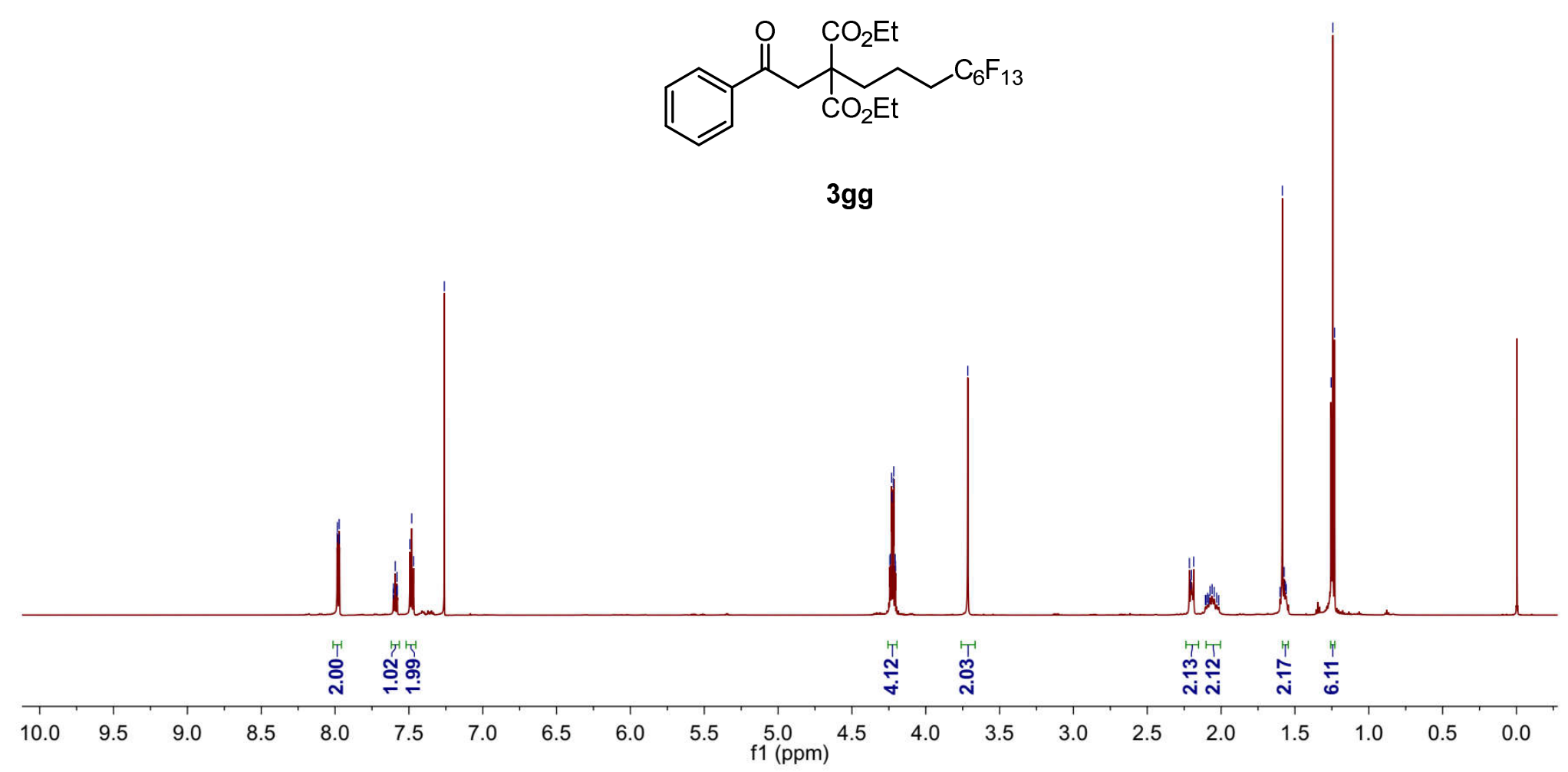




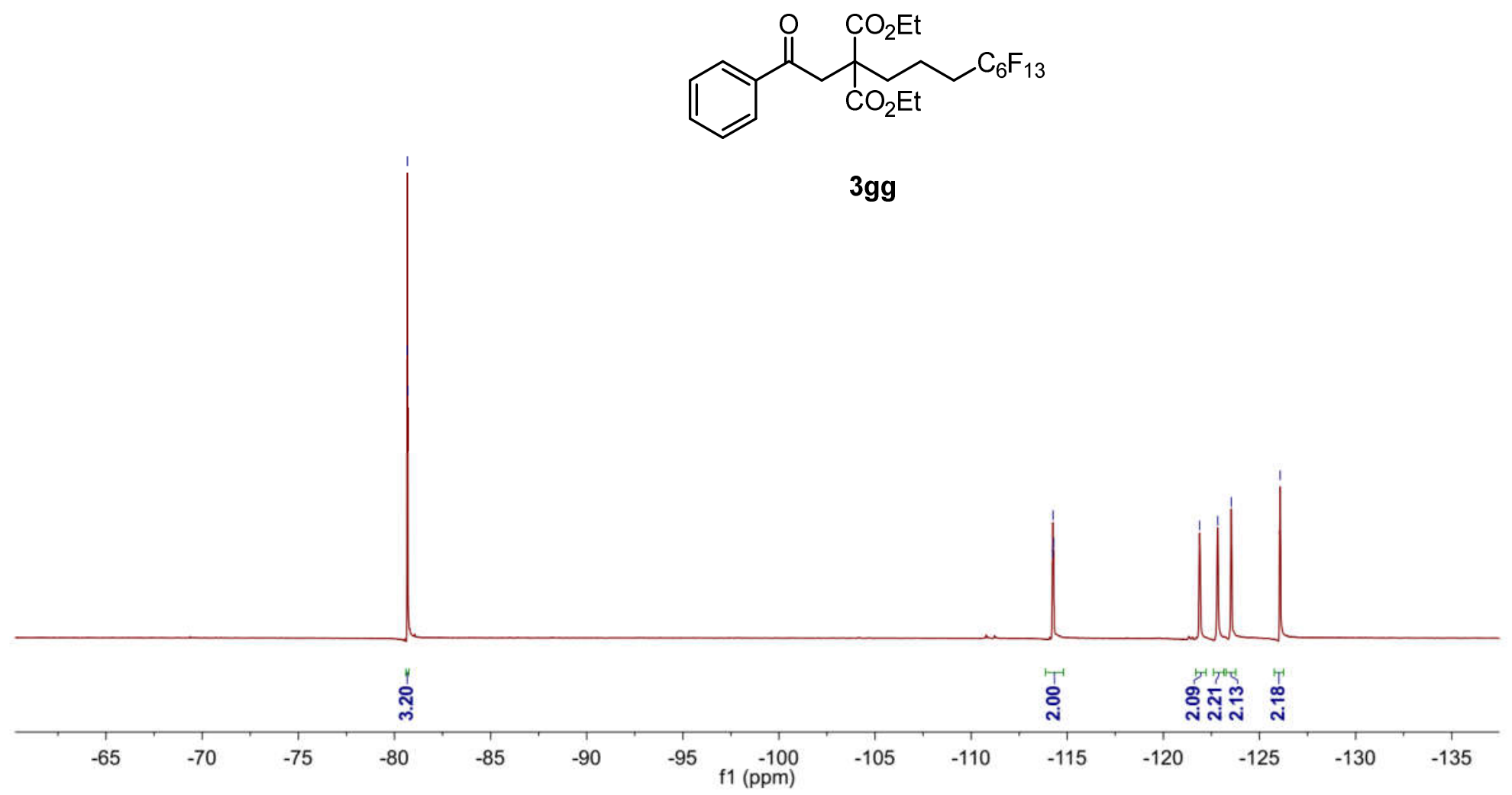




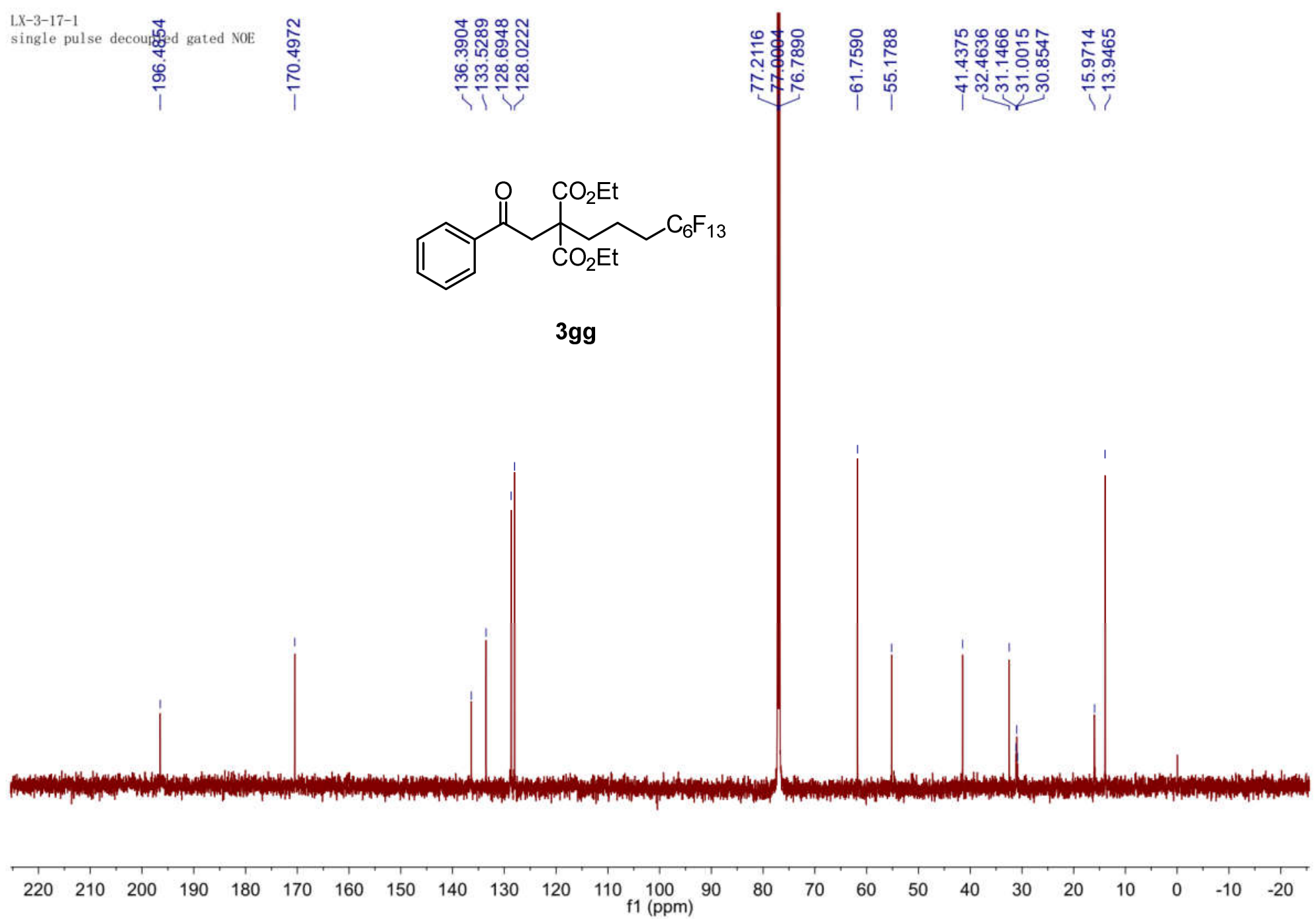




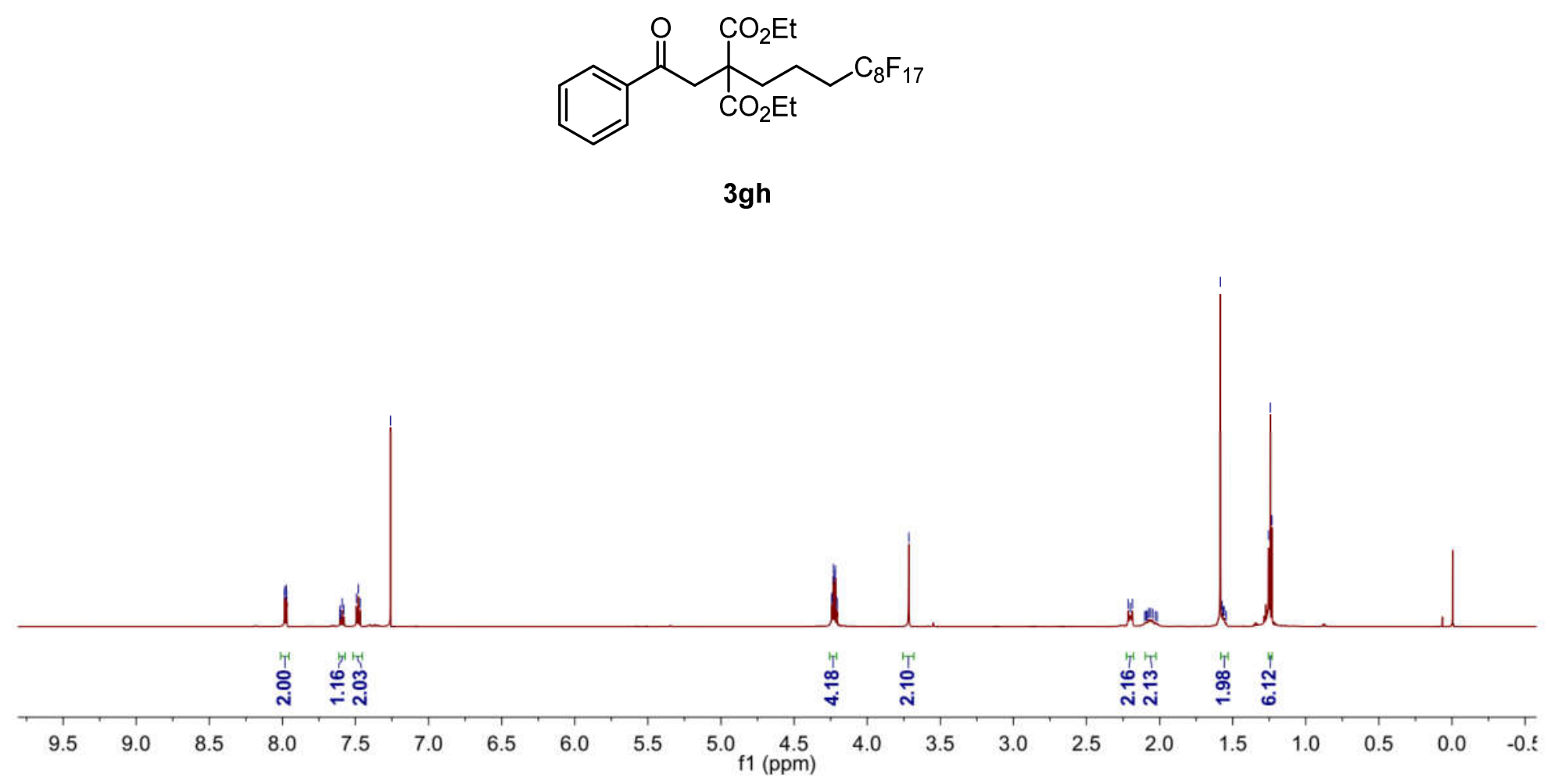


LX-3-17-2

single_pulse

웅웡웡

i

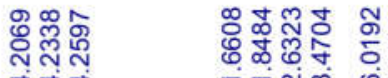

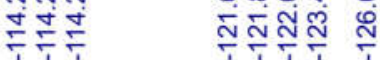

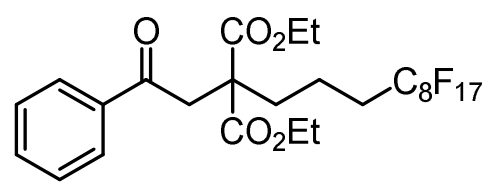

$3 g h$

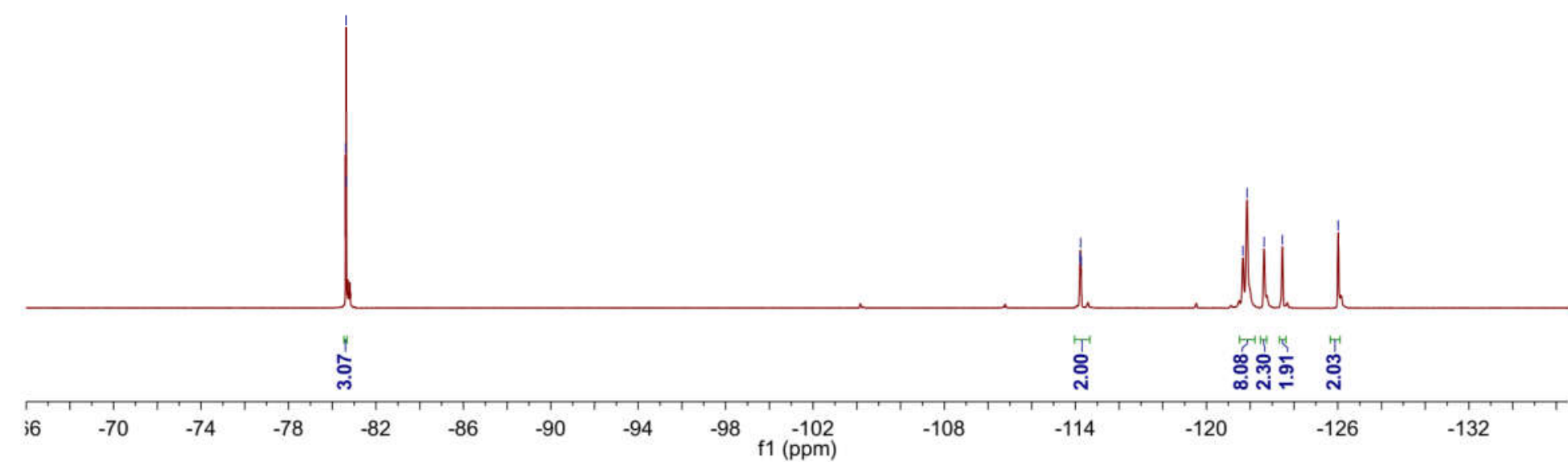




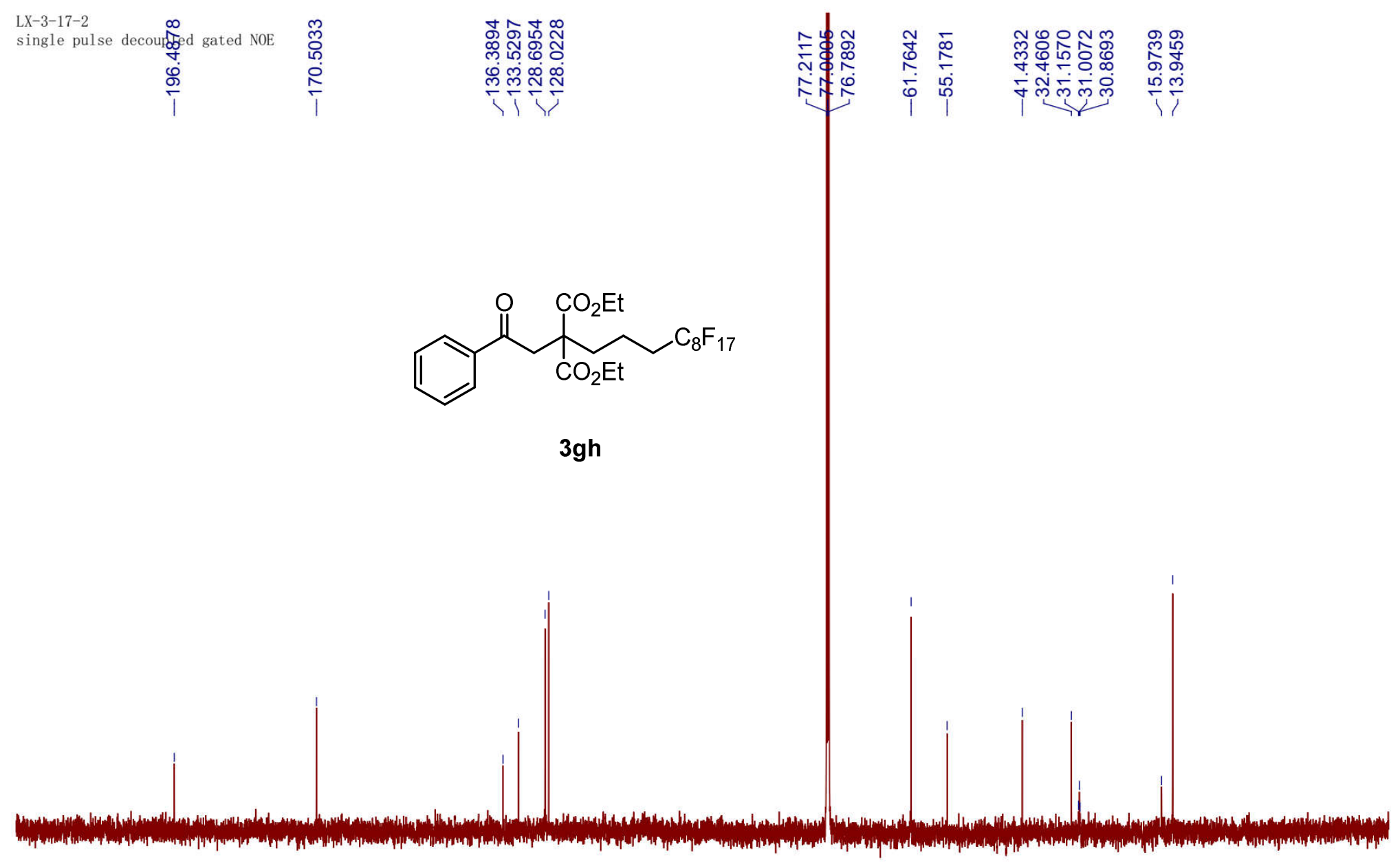

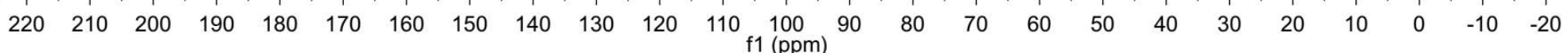




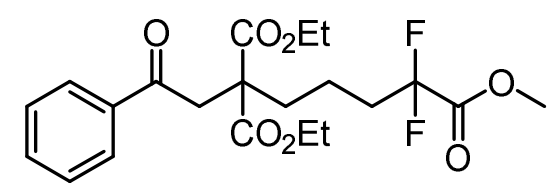

$3 g i$

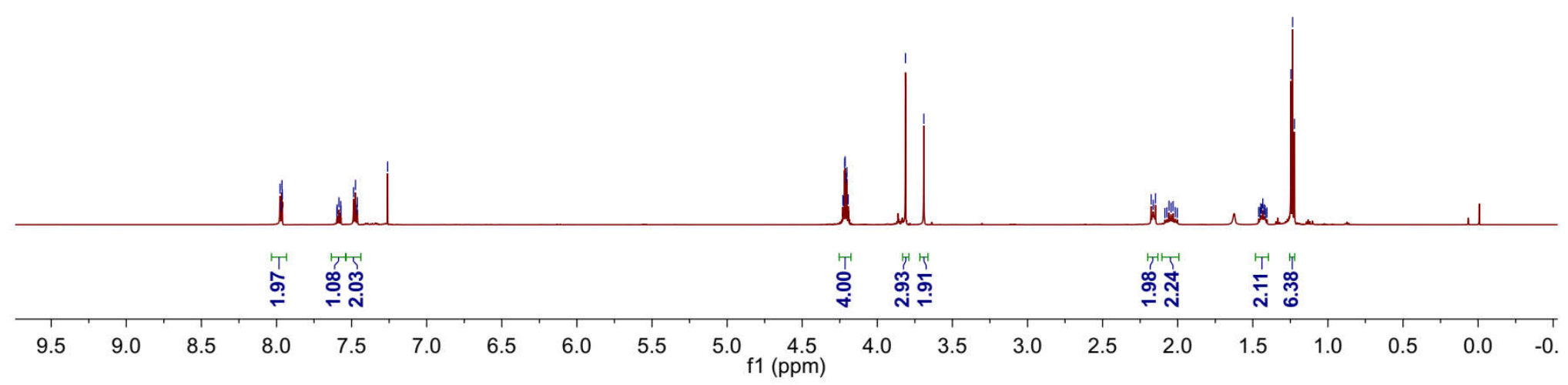




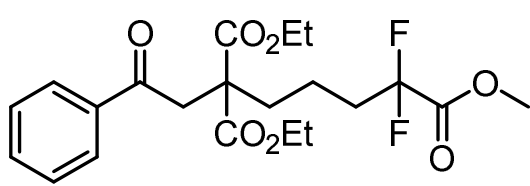

$3 g i$

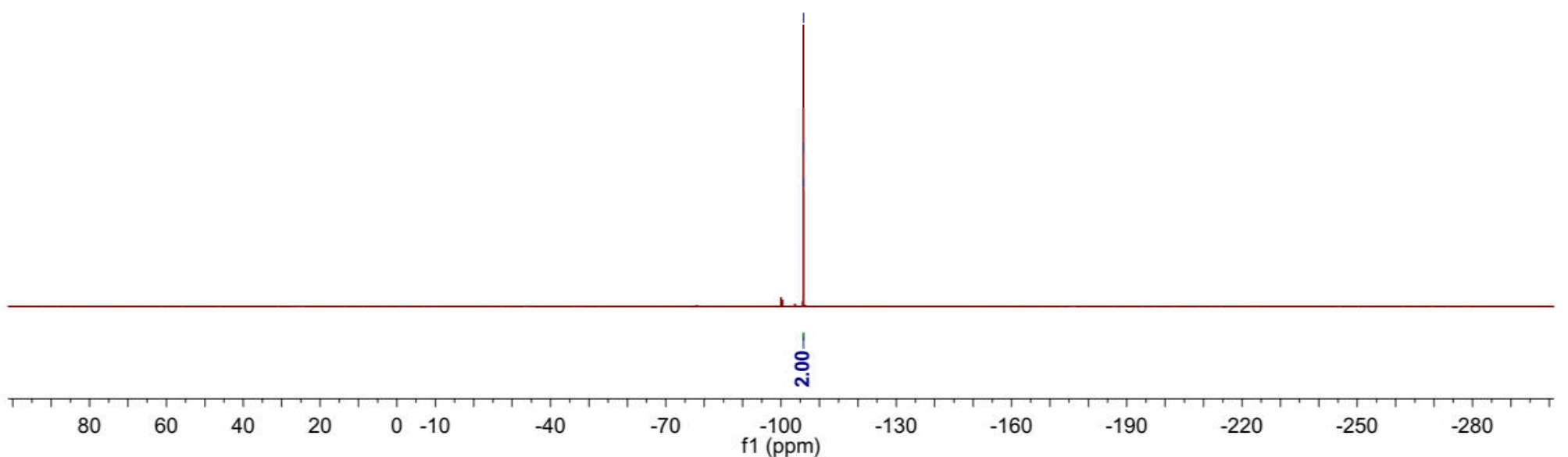




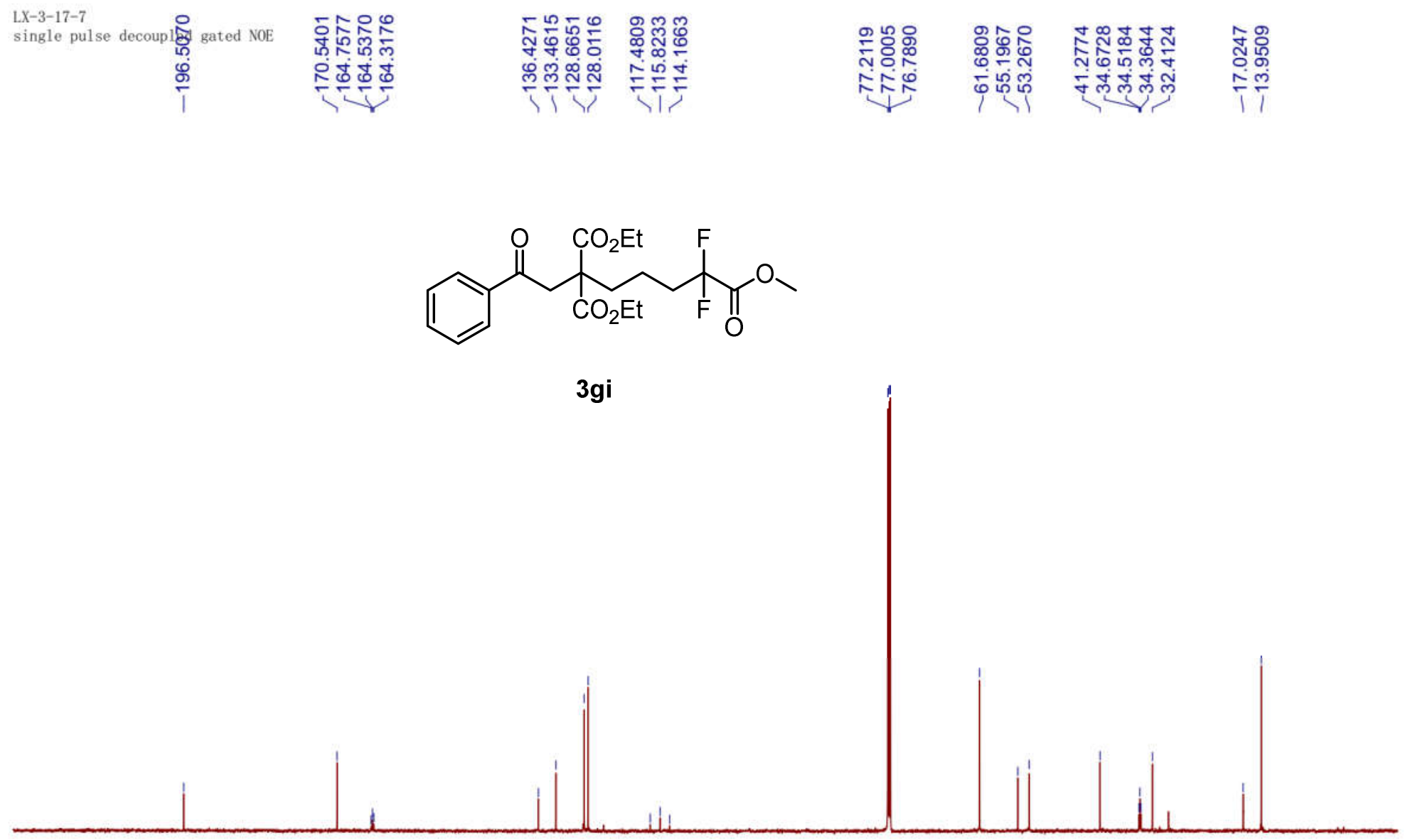

$\begin{array}{lllllllllllllllllllllll}220 & 210 & 200 & 190 & 180 & 170 & 160 & 150 & 140 & 130 & 120 & 110 & 100 & 90 & 80 & 70 & 60 & 50 & 40 & 30 & 20 & 10 & 0\end{array}$ 